University of Louisville ThinkIR: The University of Louisville's Institutional Repository

Electronic Theses and Dissertations

$12-2015$

\title{
Novel biomechanical test method for cancellous bone screws and screw augmentation with cement.
}

Kevin Lancaster

University of Louisville

Follow this and additional works at: https://ir.library.louisville.edu/etd

Part of the Biomedical Engineering and Bioengineering Commons

\section{Recommended Citation}

Lancaster, Kevin, "Novel biomechanical test method for cancellous bone screws and screw augmentation with cement." (2015). Electronic Theses and Dissertations. Paper 2284.

https://doi.org/10.18297/etd/2284

This Master's Thesis is brought to you for free and open access by ThinkIR: The University of Louisville's Institutional Repository. It has been accepted for inclusion in Electronic Theses and Dissertations by an authorized administrator of ThinkIR: The University of Louisville's Institutional Repository. This title appears here courtesy of the author, who has retained all other copyrights. For more information, please contact thinkir@louisville.edu. 


\title{
NOVEL BIOMECHANICAL TEST METHOD FOR CANCELLOUS BONE SCREWS
} AND SCREW AUGMENTATION WITH CEMENT

\author{
By \\ Kevin Lancaster \\ B.S., University of Louisville, 2014

\begin{abstract}
A Thesis
Submitted to the Faculty of the

University of Louisville

J.B. Speed School of Engineering

As Partial Fulfillment of the Requirements

For the Professional Degree
\end{abstract} \\ MASTER OF ENGINEERING \\ Department of Biomedical Engineering
}

December 2015 


\section{NOVEL BIOMECHANICAL TEST METHOD FOR CANCELLOUS BONE SCREWS}

AND SCREW AUGMENTATION WITH CEMENT

Submitted By:

Kevin Lancaster

A Thesis Approved On

\section{Date}

By the Following Reading and Examination Committee

Michael Voor, Thesis Director

\section{Gail Depuy}

Thomas Roussel 


\section{ACKNOWLEDGEMENTS}

I'd like to thank my loving parents, who helped me get through this wild ride called college in more ways than one. Thanks to my freakishly smart thesis director Dr. Voor who kept me on track during this long and sometimes stressful thesis project.

Special thanks to Lonnie Douglas for his guidance on cement mixing and how to not permanently cement my hands together.

Thanks to Dr. Depuy who helped me become a pro at Minitab statistics, as well as serving on my committee. And thanks to DOCTOR Tommy Roussel for also serving committee duties as well as being the coolest doctorate graduate you'll ever meet. 


\begin{abstract}
It is often difficult to achieve adequate screw fixation for plate constructs in fractures with poor quality bone or in metaphyseal bone with extensive bone loss or comminution[7][17]. Furthermore, rescuing or augmenting failed screw fixation in inadequate bone with various cement products has yielded mixed results when tested with pure axial pullout loading[20][21]. In most cases, plate/screw constructs experience both vertical (translational) and horizontal (pullout) forces during physiologic loading[17][20][21]. The increased use of locked screws in plate constructs has also changed the loading patterns of bone screws. For this study, a novel "toggle-out" testing method was developed to more realistically simulate in-vivo loading of screw-plate constructs. Our objective was to compare the fixation of locked and non-locked screws in simulated cancellous bone of three different densities and to determine the effectiveness of augmentation of the screw fixation using either PMMA or a resorbable Calcium Phosphate cement in both stripped and oversized screw holes.

Polyurethane foam blocks of $12.5,20$, and $30 \mathrm{lb} / \mathrm{ft}^{3}$ densities representing ostoporotic, normal, and high density cancellous bone respectively (Sawbones, Pacific Research Laboratories, Vashon Island, WA) were used as the bone surrogate for this study. Holes were drilled into the blocks perpendicular to a single face for placement of screws. All screws tested were $4 \mathrm{~mm}$ diameter, 32, 34, or $36 \mathrm{~mm}$ length stainless steel cancellous bone screws (Stryker, Mahwah, NJ). The holes were either $2.5 \mathrm{~mm}$ diameter pilot holes, $4.0 \mathrm{~mm}$ diameter (to simulate a stripped screw hole), or $12 \mathrm{~mm}$ diameter (bone loss / void). In the $4 \mathrm{~mm}$ stripped holes and the $12 \mathrm{~mm}$ holes, various cements were
\end{abstract}


used to augment the screw fixation. The cements used were PMMA (Simplex, Stryker) and Calcium Phosphate Cement (Trabexus, Vivorté). After placement of the screws or after the cement had set for 24 hours, the blocks were mounted on a load frame (MTS Corp) for cyclic testing. The load fixture allowed screws to be configured either as locked screws or non-locked screws with respect to the plate. Along with cyclic transverse loading, a constant axial pullout force of $20 \mathrm{~N}$ was applied to each screw during testing. Cyclic "toggle" loading was applied for 1000 cycles at each of $\pm 25, \pm 50, \pm 100$, and \pm 200 $\mathrm{N}$, or until failure by pullout or screw breakage. The average total displacement (positive and negative combined) value for each test was recorded over the last ten load cycles.

Under all conditions, the locked screws exhibited significantly less displacement than the non-locked screws $(\mathrm{p}<0.05)$. The locked screws also had fewer failures due to either pullout or screw breakage than did the non-locked screws. Screws placed in 12 $\mathrm{mm}$ holes augmented with cement of any kind performed better than tightly fixed controls in low density bone and also in higher density bone (fewer failures and less displacement, $\mathrm{p}<0.05)$. For $12 \mathrm{~mm}$ holes, it was found that both cement types were effective at augmenting screws and resulted in mechanical performance similar to tight screws in only bone material. In stripped holes augmented with both types of cement, the performance of augmented screws was not significantly different than tight screws when the locking plate was used.

The novel testing model used in this study revealed differences between locking and non-locking plate/screw constructs across a spectrum of bone qualities and defect conditions. To be able to compare results directly across groups, non-locking type cancellous screws were used in every case. A special locking plate fixture allowed the 
screws to be set up as locked screws for half of the test conditions. Locking screws exhibited less displacement than non-locking screws across all test samples $(\mathrm{P}=0.00)$. This study therefore supports the use of a locking-style cancellous screw in poor quality bone or when cement augmentation of large holes is warranted. The behavior in stripped holes was quite interesting and erratic. The non-locking screws easily pulled out in most cases, but the locking screws were able to survive more cycles. Also, the higher density bone made cement augmentation to prevent pullout more difficult because the cement (especially $\mathrm{CaP}$ ) does not interdigitate with the high density material. Cement augmentation of large defects and stripped holes in poor quality bone has the potential to be successful regardless of the type of cement used because locking screws were significantly more stable than non-locking screws. 


\section{TABLE OF CONTENTS}

Approval Page .........................................................................

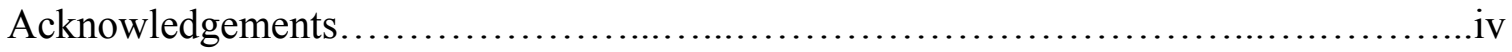

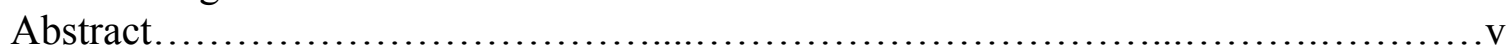

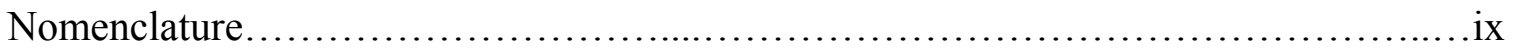

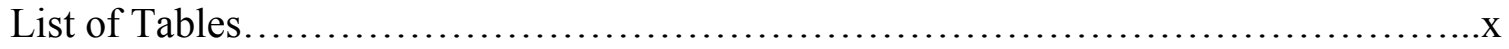

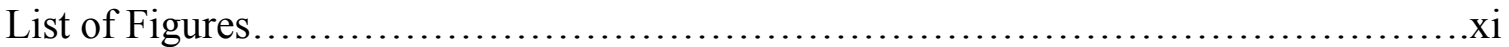

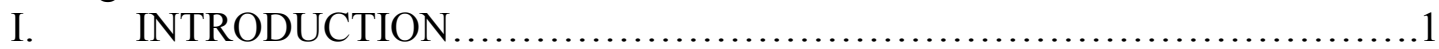

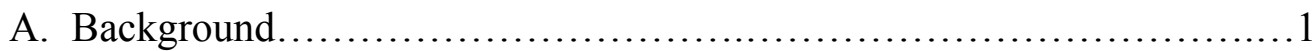

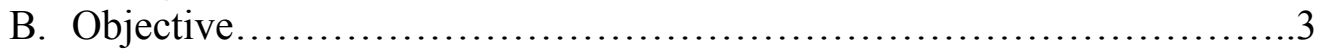

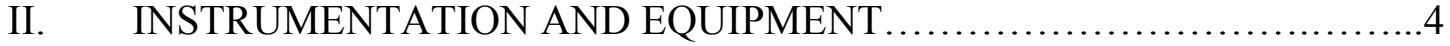

A. Equipment Setup .................................................... 4

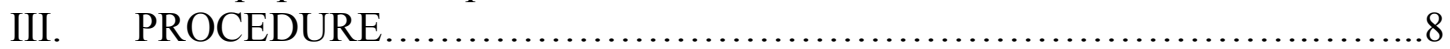

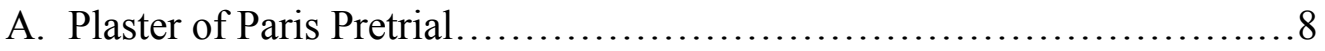

B. Cement and Sample Preparation...................................... 11

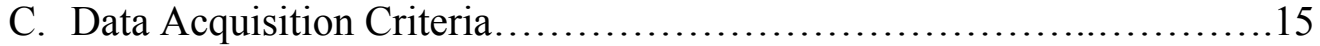

D. CaP "test" and "retest" Samples.................................... 18

E. Failure Mode under Locking Plate...................................23

F. Inherent Displacement System Error...............................25

G. Theoretical Screw Bending Validation..............................27

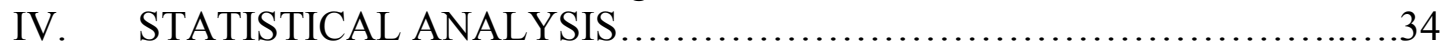

A. Cycles Survived......................................................

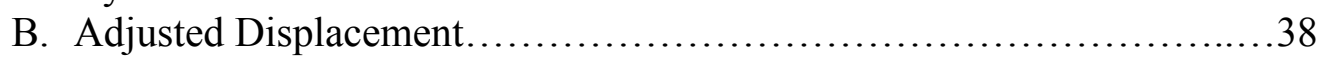

C. Load Strength..........................................................

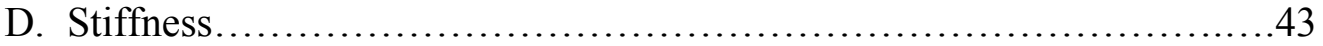

E. Cycles Survived Round Two.....................................47

F. Adjusted Displacement Round Two................................48

G. Load Strength Round Two........................................49

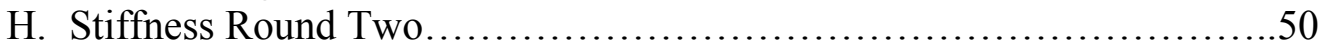

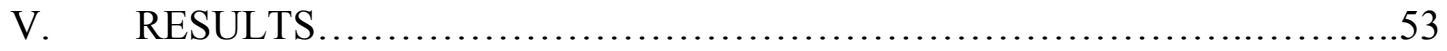

A. Control Group No Cement................................................53

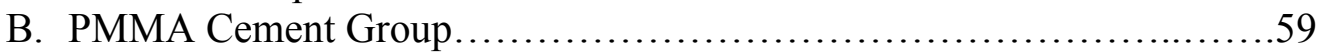

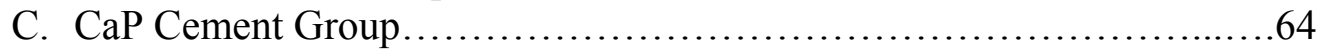

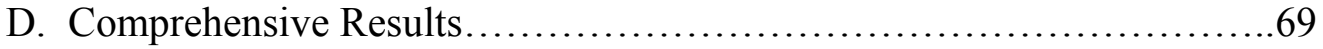

E. Post-Hoc Comparisons.............................................

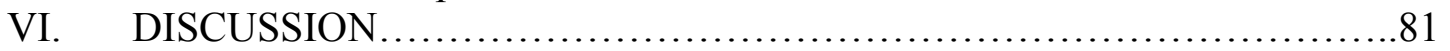

A. Unique Screw Pull-out Method.......................................... 81

B. Cancellous versus Cortical Screw Types............................. 82

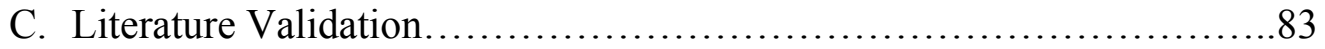

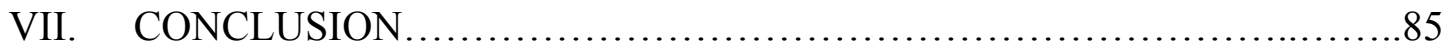

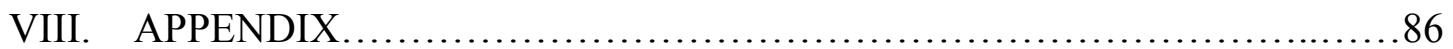

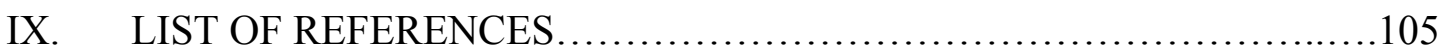

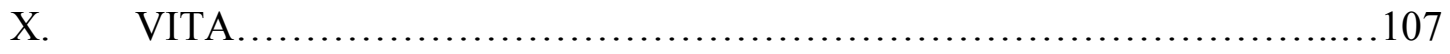




\section{NOMENCLATURE}

PMMA = Polymethyl Methacrylate bone cement

$\mathrm{CaP}=$ Calcium Phosphate bone cement

MTS $=$ Mechanical Testing System

PLC $=$ Posterolateral Corner

$\mathrm{N}=$ Newton

$\mathrm{OD}=$ Outer Diameter

ANOVA $=$ Analysis Of Variance

$\delta=$ Max Deflection

$\mathrm{W}=$ Point Load

$\mathrm{M}=$ Moment

$\mathrm{L}=$ Length

$\mathrm{E}=$ Modulus of Elasticity

$\mathrm{I}=$ Moment of Inertia

$\lambda=$ Box-Cox Transformation Coefficient 


\section{LIST OF TABLES}

Table I. Parameters for Cyclic Testing. ............................................. 8

Table II. Slop Displacement Values per Load Level..................................26

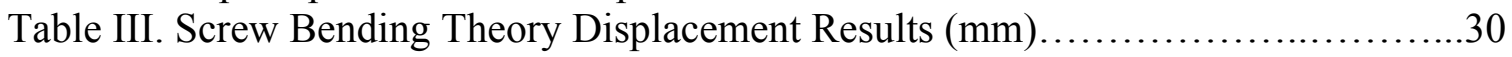

Table IV. Notable Outliers from Cycles Survived Response........................ 38

Table V. Notable outlier observations from adjusted displacement response.............40

Table VI. Notable outlier observations from Load Strength response...................43

Table VII. Notable outlier observations from Stiffness response.......................45

Table VIII. Notable outlier observations across all models, first round..................45

Table IX. Notable outliers for Cycles Survived response, round two...................48

Table X. Notable outliers for Load Strength response, round two....................49

Table XI. Notable outliers for Stiffness, round two..................................50

Table XII. Control, no cement Low Density..................................... 87

Table XIII. Control, no cement Medium Density.................................88

Table XIV. Control, no cement High Density...................................... 89

Table XV. PMMA Cement Low Density...........................................90

Table XVI. PMMA Cement Medium Density....................................91

Table XVII. PMMA Cement High Density........................................ 92

Table XVIII. CaP Cement Low Density...........................................93

Table XIX. CaP Cement Medium Density.......................................94

Table XX. CaP Cement High Density.............................................. 95

Table XXI. Displacement per Load Level Low Density Control........................96

Table XXII. Displacement per Load Level Medium Density Control...................97

Table XXIII. Displacement per Load Level High Density Control......................98

Table XXIV. Displacement per Load Level Low Density PMMA......................99

Table XXV. Displacement per Load Level Medium Density PMMA................... 100

Table XXVI. Displacement per Load Level High Density PMMA......................101

Table XXVII. Displacement per Load Level Low Density CaP....................... 102

Table XXVIII. Displacement per Load Level Medium Density CaP...................103

Table XXIX. Displacement per Load Level High Density CaP.......................104 


\section{LIST OF FIGURES}

Pigure 1: Front view of the testing apparatus with parts labeled........................4

Figure 1: Front view of the testing apparatus with parts labeled.......................

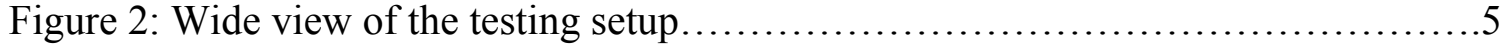

Figure 3: Up close view of the Custom plate......................................6

Figure 4: Illustration and subsequent view of the basic test setup for this study...........7

Figure 5: Plaster of Paris Pre-trial: General linear model ANOVA results...............10

Figure 6: Visualization of the two custom locking plates used throughout the study......11

Figure 7: Real time graph of Force and displacement over time..................... 17

Figure 8: General linear model ANOVA results for "modified hand" vs "drill"..........19

Figure 9: Low Density $12 \mathrm{~mm}$ (large) hole Calcium phosphate samples.................21

Figure 10: General linear model ANOVA results for Hand vs Drill RETEST ...........22

Figure 11: A visualization of locking and traditional smooth head screws...............24

Figure 12: Beam bending theory values and sketch, single fixed end..................27

Figure 13: Beam bending theory values and sketch, double fixed end..................29

Figure 14: A comparison of the force displacement curves, screw bending theory........33

Figure 15: Standardized residuals for general linear model ANOVA, cycles survived...36

Figure 16: Box-Cox transformation, residuals for ANOVA, cycles survived.............36

Figure 17: Significant factor interactions for the cycles survived response...............37

Figure 18: Standard residuals, fitted values for adjusted displacement ANOVA.........38

Figure 19: Residual plots from general linear model ANOVA, adjusted displacement...39

Figure 20: ANOVA results and P-values, adjusted displacement........................39

Figure 21: ANOVA results after removing factors, adjusted displacement..............40

Figure 22: Probability plot from ANOVA for Load Strength........................41

Figure 23: Residual plots for Load Strength response.............................41

Figure 24: ANOVA, box-cox transformation for Load Strength response...............42

Figure 25: Residual plots of the Stiffness response from ANOVA......................43

Figure 26: Residual plots and P-values for Stiffness ANOVA.........................44

Figure 27: ANOVA results for Stiffness response after removing factors..............44

Figure 28: ANOVA results for Cycles Survived response after removing outliers.......47

Figure 29: ANOVA results for Adjusted Displacement after removing outliers..........48

Figure 30: ANOVA results for Load Strength after removing outliers.................49

Figure 31: ANOVA results for Stiffness response after removing outliers..............50

Figure 32: Final ANOVA results for all four factors with all outliers removed...........51

Figure 33: All four responses for control group, low density.......................54

Figure 34: Displacement averages at $100 \mathrm{~N}$ and stiffness for low density control...........55

Figure 35: Adjusted displacement per load level, low density control..................55

Figure 36: All four responses for control group, medium density....................56

Figure 37: Displacement averages at $100 \mathrm{~N}$ and stiffness for medium density control....56

Figure 38: Adjusted displacement per load level, medium density control...............57

Figure 39: All four responses for control group, high density .......................57

Figure 40: Displacement averages at $100 \mathrm{~N}$ and stiffness for high density control........58

Figure 41: Adjusted displacement per load level, high density control...................58

Figure 42: All four responses for PMMA cement group, low density..................59

Figure 43: Displacement averages at 100N and stiffness for low density PMMA.......60

Figure 44: Adjusted displacement per load level, low density PMMA.................60 
Figure 45: All four responses for PMMA cement group, medium density..............61

Figure 46: Displacement averages at $100 \mathrm{~N}$ and stiffness for medium density PMMA...61

Figure 47: Adjusted displacement per load level, medium density PMMA..............62

Figure 48: All four responses for PMMA cement group, high density..................62

Figure 49: Displacement averages at 100N and stiffness for high density PMMA........63

Figure 50: Adjusted displacement per load level, high density PMMA..................63

Figure 51: All four responses for $\mathrm{CaP}$ cement group, low density.....................64

Figure 52: Displacement averages at $100 \mathrm{~N}$ and stiffness for low density CaP............65

Figure 53: Adjusted displacement per load level, low density CaP ....................65

Figure 54: All four responses for CaP cement group, medium density.................66

Figure 55: Displacement averages at $100 \mathrm{~N}$ and stiffness for medium density CaP.......66

Figure 56: Adjusted displacement per load level, medium density CaP ................67

Figure 57: All four responses for CaP cement group, high density....................67

Figure 58: Displacement averages at $100 \mathrm{~N}$ and stiffness for high density CaP.........68

Figure 59: Adjusted displacement per load level, high density CaP ..................68

Figure 60: Displacement at 200N low density all cement types, non-stripped holes......69

Figure 61: Displacement at 200N medium and high density, non-stripped holes.........70

Figure 62: Displacement at 100N low density all cement types, stripped holes...........71

Figure 63: Displacement at 100N medium and high density, stripped holes............. 72

Figure 64: Stiffness results for all cement groups, low density ......................73

Figure 65: Stiffness results for all cement groups, medium and high density.............74

Figure 66: Cycles survived averages for all cement groups, all hole types................76

Figure 67: Tukey Pairwise test for low density 100N Adjusted Displacement............78

Figure 68: Tukey Pairwise test for low density 200N Adjusted Displacement............79

Figure 69: Tukey Pairwise fitted means for low density stiffness response..............80

Figure 70: Unique pull out method for high density $\mathrm{CaP}$ samples....................81 


\section{INTRODUCTION}

\section{A. Background}

The use of bone screws to stabilize orthopedic plates is a widely applied technique to aid in the healing process for common fractures. Plates and/or screws can be secured on long bones of arms and/or legs as well as the pelvis and the spine.

Complications may arise during these procedures due to osteoporosis, which is often an underlying cause of fractures, specifically with proximal humeral fractures [5].

Osteoporosis, along with other pathological causes, exhibits poor quality cancellous bone and makes it difficult for orthopedic surgeons to attach the plates using screws. Low bone density at the screw-bone interface increases the risk of screw loosening or pull-out, and results in poor fixation of the plate and no fracture site stability.

The widely accepted solution to combat low bone density is to fill voids within weak cancellous bone adjacent to the screws with Polymethylmethacrylate (PMMA) cement [10]. Cancellous, rather than cortical bone is recommended for augmentation due to its porosity; cortical bone has less empty space with which the cement can interdigitate and create a secure connection [9]. A stronger bone - cement interface corresponds directly to stronger shear strength while holding the plate in place. Studies have shown significantly increased pull out strength using PMMA than without [3][6][12][24]. In a 
case study of 21 patients all using fenestrated pedicle screws with PMMA cement, all 21 reported no loosening or pulling out of screws after 36 months [7]. However, PMMA has its disadvantages such as poor biocompatibility, exothermic polymerization and nonresorbability (Stadelmann et al., 2010; Larsson et al., 2012)[14;15].

The plate screw interface can be either locking or non-locking depending on the presence of threaded holes within the plate. A non-locking screw head gets its strength from friction along the head of the screw and compression across the entire plate. Large compressive forces may adequately hold the plate in place but it reduces blood flow to the affected area and can cause necrosis of bone tissue under the plate. Locking screws create a fixed angle of $90^{\circ}$ between screw and plate therefore abandoning the compressive properties of the non-locking scheme. This is advantageous due to increased pull out forces and lateral stability as well as a marked decrease in trauma to the bone itself. Additionally, fully threaded (locking) lag screws have shown a maximum torque more than 3 times greater than partially threaded (non-locking) lag screws [2].

However, despite the apparent advantages of locking screws, non-locking screws are still widely used in clinical applications due to their compressive nature. In the case of some transverse fractures, when a clean break perpendicular to the long axis of the bone occurs, a compressive force normal to the fracture line can greatly assist in the healing process. The gold standard of plate fixation involved a non-locking plate with inwardslanted grooves on either side of the median line of the fracture which caused screw heads to slide towards the fracture site. As the screws slide inward they pull either side of the bone thereby pushing the two sides together, promoting quicker callus formation and ultimately a faster healing process. 


\section{B. Objective}

The purpose of this study was to test the efficiency and strength of a new Calcium-Phosphate bone cement $(\mathrm{CaP})$ and compare it to the industry standard PMMA. The null hypothesis was that there would be no difference between the CaP cement and the PMMA cement, and that the cement augmentation would be more stable than a simulated loose screw with no cement. If this was the case, CaP may replace PMMA in some cases due to its ability to resorb into the patient's natural bone.

This study further aims to develop a novel method of simulating the forces involved that lead to clinical loosening of plates and screws from cancellous bone. In the past PMMA cement has shown to be extremely resilient to shear force [21][24], yet screw loosening / pulling out has still been reported for some cases. In a recent study exploring patients with damaged PLC (corrected with PMMA) 8 out of 12 suffered from screw loosening one year post op [11]. Using a more comprehensive cyclic pullout testing structure, this study aims to reveal any weaknesses in a cement/screw interface that may have been previously overlooked. Rather than simply loading samples vertically $[19][20][21]$ or torsionally in a single direction $[22][23][25]$, this study will cyclically load samples and have a constant pullout force to create shear stress and axial pullout stress at the same time. This method will more closely simulate the forces acting on the plates and screws associated with everyday tasks such as walking or climbing stairs. 


\section{INSTRUMENTATION AND EQUIPMENT}

\section{A. Equipment Setup}

This project implemented an MTS Bionix Test System with a 5000N (1100 lbs.) capacity load cell (model 661 19E-01, MTS Corp, Eden Prairie, MN). Before testing began, an interface had to be created. It consisted of three sections, a bottom, top and adjacent. A $19^{3 / 16 "} \times 1 \frac{11 / 2 "}{}$ x 1/2" stainless steel plate was attached to the bottom MTS interface as the base (1) of the device. $8^{1 / 16 "}$ long $1 / 2$ " diameter threaded support rods (2) were inserted on each end of the base plate. Just above the base plate and also secured on either end by the $1 / 2 "$ rods was two identical $18 " \times 1 \frac{1}{2} " \times 1 / 2 "$ plates, which serve as the bottom and top block clamps (3). During testing the foam blocks were placed between these two

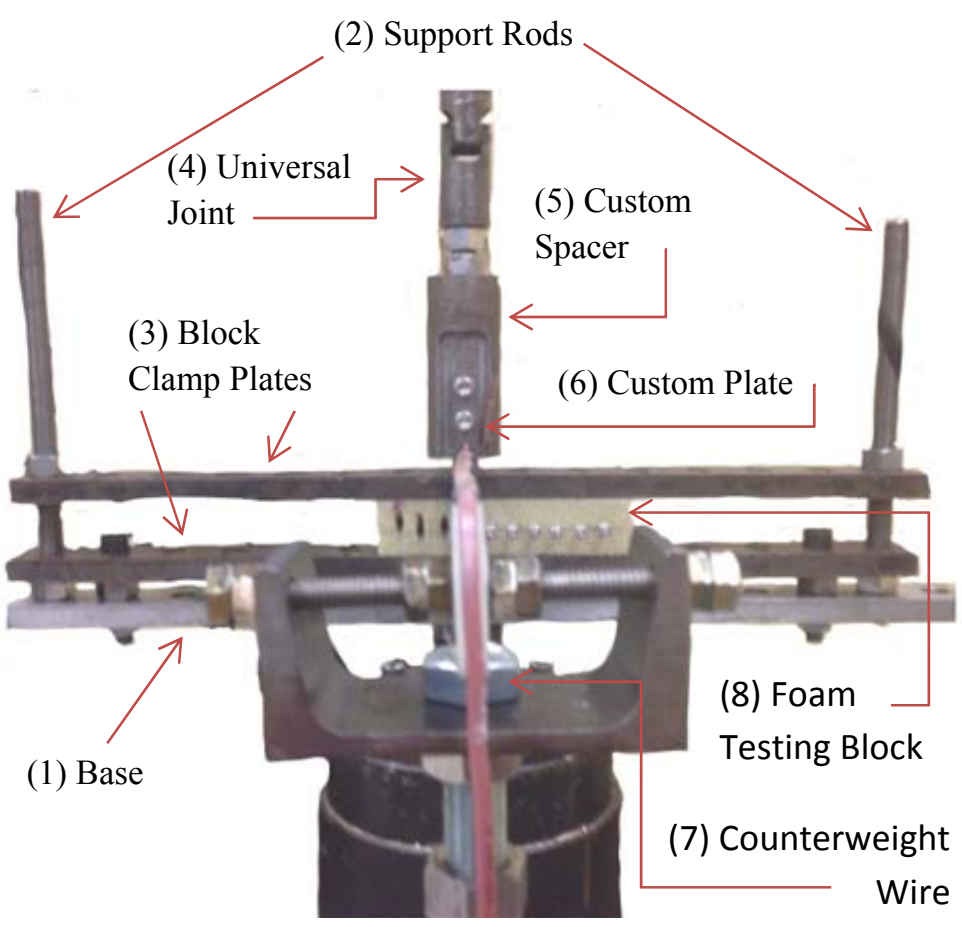

Figure 1: Front view of the testing apparatus with parts labeled. Part dimensions are detailed fully in the text. 
identical plates to be held firmly in place.

The top section played two roles by both holding the custom plate at the correct height to attach to the screws in the foam testing block (8), and allowing the plate to swivel freely back and forth / side to side in order to connect and remove from the plate before and after testing without disrupting the block. A $3^{3 / 8}, 1$ " diameter universal joint (4) descended from the top followed by a $3^{9 / 16 "}$ long, $1 \frac{1 / 2}{2}$ " diameter custom iron spacer (5). These were both spaced out by two $1 / 2$ " nuts. This section was threaded into the bottom of the $5000 \mathrm{~N}$ load cell, which rests at the top interface of the MTS machine. The custom iron spacer held the most vital part of the entire fixture: the custom plate (6). This was a $4 " x$ 1/2" x 3/16" aluminum plate with a slot that was specifically milled to fit the head of the cancellous screws used throughout this study. It also had a

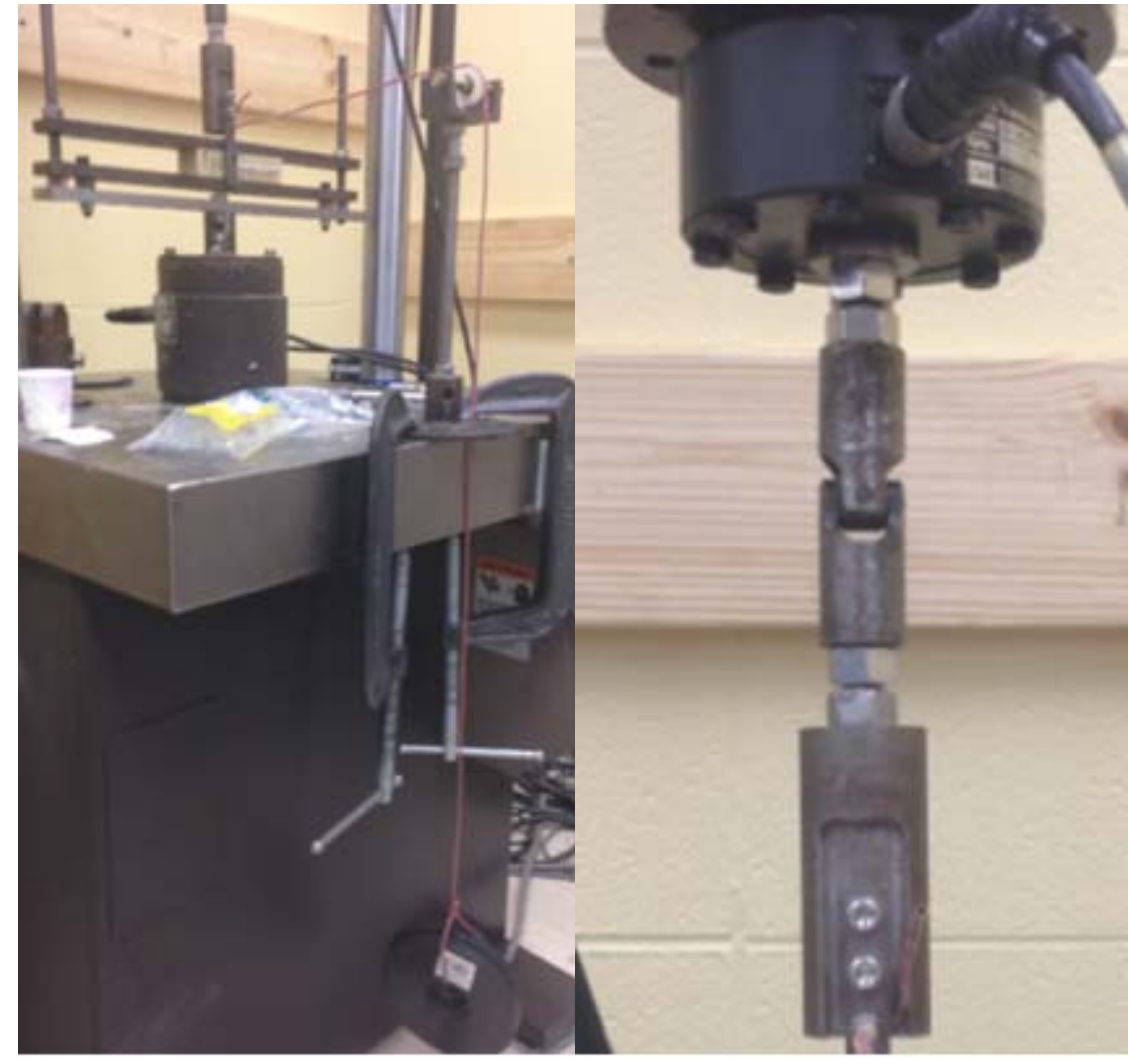

Figure 2: Wide view of the testing setup with counterweight and large Cclamps (left) and MTS Strain Gage / universal joint interface at the top of the setup (right). 
$\sim 0.108$ " hole on either side of the slot to which the locking or nonlocking plate can be secured as well as a 0.145 " hole through which the 0.125 " counterweight wire (7) was knotted.

The adjacent section simply held the counterweight pulley system in place. Ideally, the top of the fixture would allow the wire holding the weight to rest perfectly

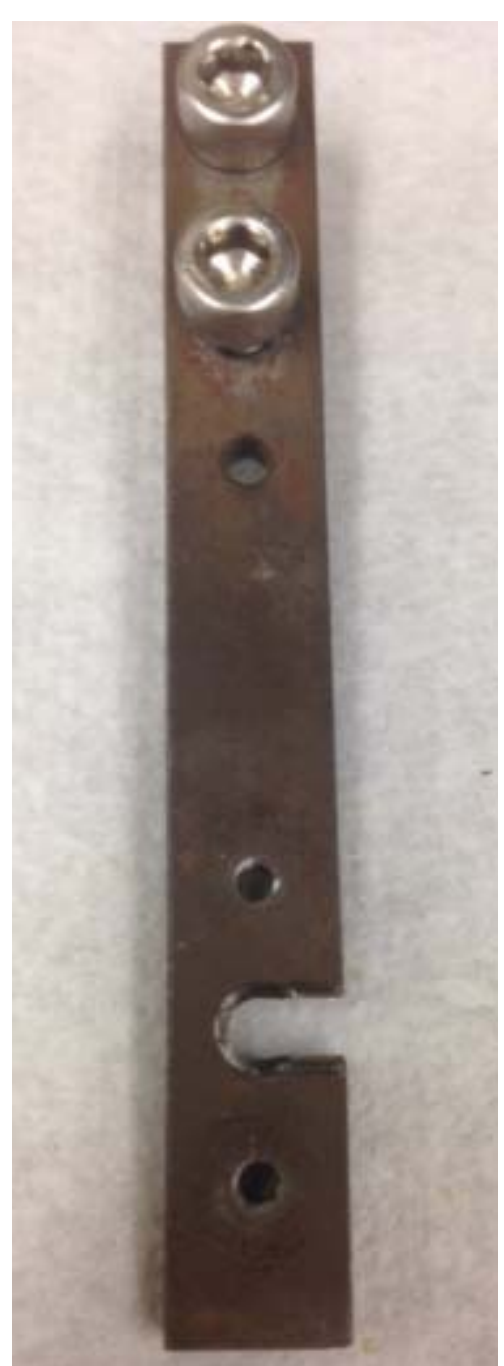

Figure 3: Up close view of the Custom plate. Screw heads fit in the slot towards the bottom and a Locking or Non-locking attachment was then secured around the head of the screw to simulate the correct screw head type. perpendicular to the custom plate connection, but a few simple trigonometric calculations compensated for irregularities. A circular base plate rested on the flat surface of the MTS machine directly in front of the custom interface, and was secured in place by two $200 \mathrm{~mm} \mathrm{C}$ clamps. A $12 \frac{1}{1 / 2}$ " threaded rod extended upward which held the 3" wide pulley system. Together, these three sections worked together to provide the framework for successful testing throughout this project.

The goal of this testing fixture was to directly apply the MTS machine's single axis of controlled force to the head of the sample screw, while also holding the foam block securely in place. Also, the fixture included the pulley system for the counterweight.

Polyurethane foam blocks were used to duplicate the consistency of human bone for this study. These 12.5, 20 , and $30 \mathrm{lbs} / \mathrm{ft}^{3}$ blocks were cut into symmetric 5.1" $\mathrm{x}$ 
$1.6 " \mathrm{x} 1.05 "(130 \mathrm{~mm} \times 40 \mathrm{~mm} \times 27 \mathrm{~mm})$ strips, which were then drilled in preparation for the screws. Each strip accommodated an entire set of ten $2.5 \mathrm{~mm}$ or $4 \mathrm{~mm}$ diameter holes, or six $12 \mathrm{~mm}$ diameter holes depending on the sample type. Before every test, the foam block was carefully placed in the center of the testing fixture and securely fastened using the tightening nuts on either side. The custom plate was then swung into place onto the head of the screw and the appropriate attachment plate was connected.

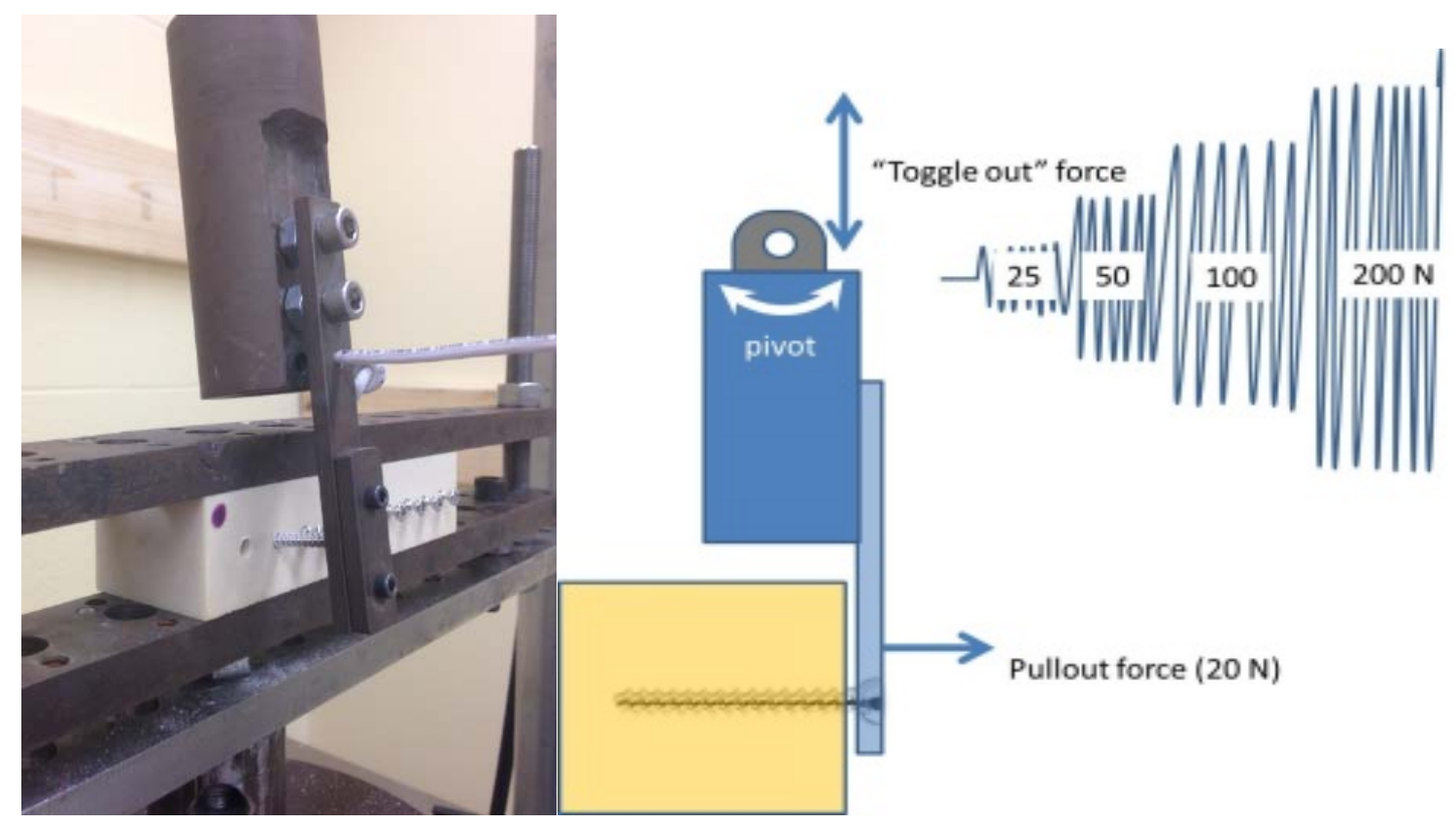

Figure 4: Illustration (right) and subsequent view (left) of the basic test setup for this study. Screws were held in the yellow foam blocks by the custom plate which was attached to the actuator of the MTS machine and cycled up and down with increasing force all while a constant pullout force of roughly $20 \mathrm{~N}$ was attempting to pull the screws directly out.

All screws used in this study were $4 \mathrm{~mm}$ diameter, 32,34 , or $36 \mathrm{~mm}$ length stainless steel cancellous bone screws (Stryker, Mahwah, NJ). The holes were either 2.5 $\mathrm{mm}$ diameter pilot holes, $4.0 \mathrm{~mm}$ diameter (to simulate a stripped screw hole), or $12 \mathrm{~mm}$ diameter (bone loss / void). In the $4 \mathrm{~mm}$ stripped holes and the $12 \mathrm{~mm}$ holes, various cements were used to augment the screw fixation. The cements used were PMMA (Simplex, Stryker) and Calcium Phosphate Cement (Trabexus, Vivorté). 


\section{PROCEDURE}

\section{A. Plaster of Paris Pre-trial}

Six large $(12 \mathrm{~mm})$ holes were cut into existing foam block and filled with plaster of Paris. In each hole, a $30 \mathrm{~mm}$ long non-locking cancellous $4 \mathrm{~mm}$ (OD) screw was placed in the hole. For each set (6 holes total) 3 screws were pushed into the plaster of Paris immediately upon filling and held in place by hand until the plaster was set. The other 3 holes were allowed to set for 30 minutes before drilling a 0.089 " hole (inner diameter) into the plaster and using a screwdriver or Allen wrench to insert the screw. After insertion, samples were left to dry at least 24 hours before testing. Each hole was tested at $\pm 25 \mathrm{~N}$ for 1000 cycles, and then increased by a factor of 2 in both directions for each set of 1000 cycles after that 3 times or until failure (table I). Screws and plates were reused for this study unless failure mode was screw head failure. Counter weight for all Plaster of Paris trials was two 2.5 lbs plates at the end of the wire which pulls at an equivalent of $3.92 \mathrm{lbs}$ or $17.44 \mathrm{~N}$ normal to the foam block. All trials throughout the study used the force range and cycles shown below in Table I.

Table I: Parameters for Cyclic Testing

\begin{tabular}{|c|c|}
\hline Force Range (N) & Number of Cycles \\
\hline$-25-25$ & 1000 \\
\hline$-50-50$ & 1000 \\
\hline$-100-100$ & 1000 \\
\hline$-200-200$ & 1000 \\
\hline
\end{tabular}


Two sets (12 holes) were completed using a machined PVC pipe connector for a swivel interface and a 7-hole dual threaded locking plate at each hole. Another 2 sets were tested using the custom testing plate fixture; the first of which used the locking cover plate and the second used the non-locking cover plate to simulate a locking and non-locking screw orientation respectively. Distances from the fulcrum of the PVC pipe to the counter weight wire and from the fulcrum to the screw head were taken into account and replicated when installing the custom test plate to insure that the counter weight was pulling with the same amount of force for all four sets.

The main objective of the Plaster of Paris pre-trial was to get a feel for the testing equipment and MTS machine and also to establish which method of screw insertion was more beneficial. The initial hypothesis was that setting the screw into the liquid cement by hand would allow for better screw-cement adhesion than drilling a hole into set cement and inserting the screw from there. Wet cement, in theory, will conform to the threads of the screw before setting, optimizing the contact between screw and cement. However, the results showed the screws inserted by the drill technique displaced slightly less than the hand technique. This being said, there was no statistical difference between the two, as shown by table 2 below. Both P-values were less than 0.01 for a $99 \%$ confidence that both techniques were not statistically different. The Plaster of Paris tests determined it was safe to use either the hand set or drilled screw insertion technique for trials moving forward. 

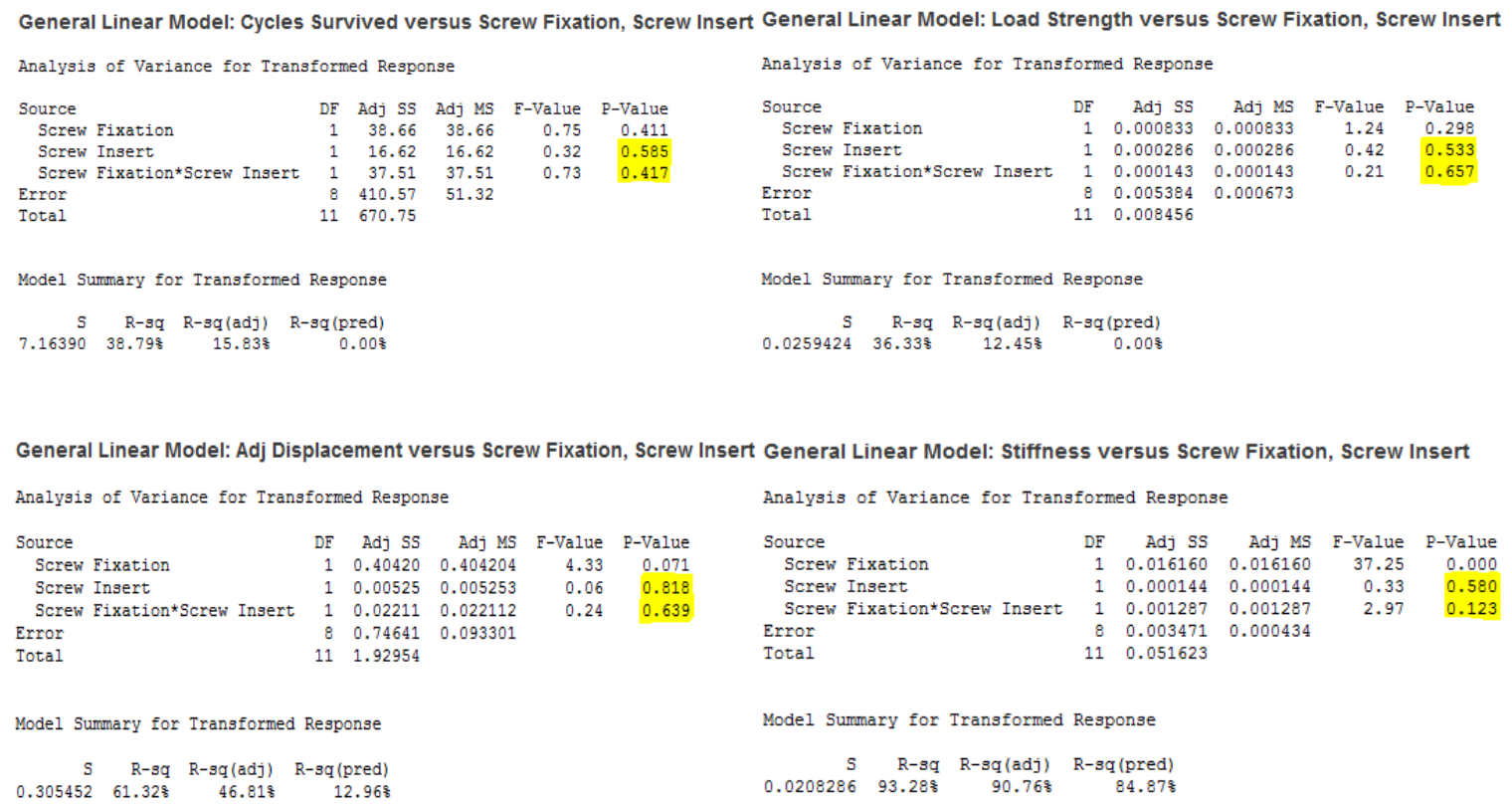

Figure 5: Plaster of Paris Pre-trial: General linear model ANOVA results for Cycles Survived (top left) Load Strength (top right) Adjusted Displacement (bottom left) and Stiffness (bottom right). All four responses showed a $\mathrm{P}$-value greater than $\mathrm{P}=0.10$ for both the Screw Insert factor (hand threaded vs drilled) and the Screw Fixation*Screw Insert factor interaction. These P-values show that the Screw Insert factor was NOT significant to the data and therefore the hand threaded and drilled insertion techniques were interchangeable for Plaster of Paris trials. This may or may not translate to other cements.

When the Plaster of Paris samples were completed, it was time to begin regular testing trials. The same cyclic protocol was used for regular samples, shown in Table I above. Also, screws were tested under the above conditions until failure, in which case the test was immediately stopped and the screw was removed from the block. If the screw survived all 4000 cycles, it was still removed to allow the custom plate to attach to the succeeding screw. The average total displacement (positive and negative combined) value per cycle for each test was recorded over the last ten load cycles.

The first group of screws tested was the control group without cement. As stated above, all locking and nonlocking samples were tested using the locking or nonlocking attachment plate respectively (see Figure 6 below). The appropriate density block of 12.5 , 
20, or $30 \mathrm{lbs} / \mathrm{ft}^{3}$ was also used for Low, Medium and High density variables respectively.

These rules pertain to all trials moving forward.
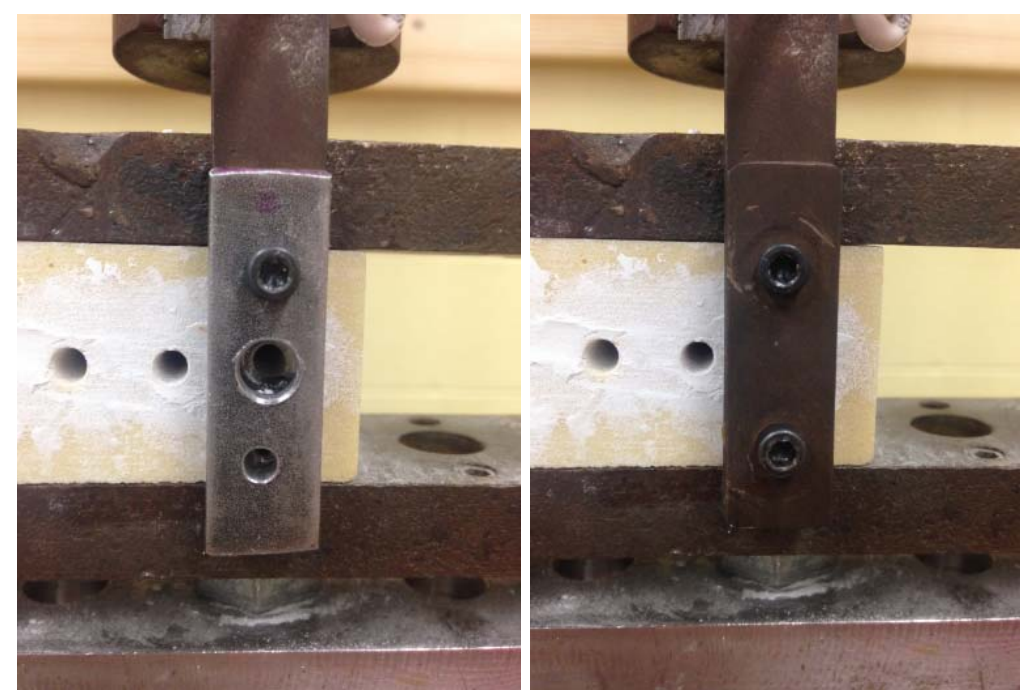

Figure 6: Visualization of the two different custom locking plates used throughout the study. Left: Nonlocking plate with an opening in the center. This plate simply keeps the head of the screw in the slot of the custom plate while allowing the screw to toggle freely during cyclic loading. Right: Locking plate without an opening. When this plate was securely tightened down on either side it puts enough pressure on the head of the screw to keep it locked at a right angle with the custom plate, simulating a locking head screw construction.

\section{B. Cement and Sample Preparation}

A special thanks to Stryker Orthopaedics and Vivorte Inc. for providing donations of PMMA and calcium phosphate cements respectively for this study.

\section{a. Control, Tight}

A drill bit equal to the inner diameter of a $4 \mathrm{~mm}$ cancellous screw, approximately $2.5 \mathrm{~mm}(0.089$ "), was used to drill holes in the polyurethane block for each sample. The screw was inserted into the block with just enough clearance for the testing plate to slide 
onto the head of the screw. Once the testing plate and/or cover plate was securely fastened, the counter weight pulley system was attached and the testing cycles began.

\section{b. Control, Stripped}

A drill bit slightly smaller than the diameter of the $4 \mathrm{~mm}$ screws was used to drill the Polyurethane block for each sample. The screw was inserted into the block with enough clearance for the testing plate to slide onto the head of the screw. Once the testing plate and/or cover plate was securely fastened the counter weight pulley system was attached and testing ensued. The nonlocking samples tested in the stripped holes tended to pull out almost immediately upon starting the testing cycles, so a starting load of about $+20 \mathrm{~N}$ was used to stabilize the screw initially before the cycles started. Otherwise, the counterweight pulled the screw completely out under the force of the weight before the test had started.

\section{c. PMMA mixing}

Simplex Polymethyl Methacrylate cement (Stryker, Mahwah, NJ) was used as the PMMA for this project. A single box (42 grams each) was mixed at a time and distributed into holes completely before a new box was opened. This was to allow all screws to be placed into the appropriate holes before the cement set. Once the PMMA hardens and was no longer a liquid, it was impossible to drill or shape in any way. The set time was 15-20 minutes after initial mixing. 


\section{d. PMMA, $12.0 \mathrm{~mm}$}

The large holes were approximately $12 \mathrm{~mm}$ in diameter. After the hole was drilled out of the Polyurethane block it was filled to the top with PMMA cement. It was important that the screws were placed in the PMMA as soon as possible after the cement was mixed and poured. Screws were inserted by hand and manually rotated as if threading the cement for optimal screw adhesion. All PMMA samples were allowed to cure for at least 24 hours prior to testing to assure the cement was fully set.

\section{e. PMMA, $4 \mathrm{~mm}$}

The same sized drill bit was used again for the PMMA stripped holes as before $(3.93 \mathrm{~mm})$. Because these holes were substantially smaller than the large holes, special care was taken to make sure the cement was distributed evenly inside the voids. Upon insertion, screws were pushed completely into place very quickly, and then manually rotated in place to even out the cement inside. All $4 \mathrm{~mm}$ hole samples were allowed to cure for at least 24 hours prior to testing.

\section{f. CaP Mixing}


The Calcium Phosphate supply came in 5cc and 3cc kits. Each kit was thoroughly mixed and placed into the desired voids before another kit was opened. This cement more closely resembled the Plaster of Paris used for pre-trial samples than the PMMA, which provided more freedom to mold the paste into the needed shape. Because $\mathrm{CaP}$ comes in much smaller package sizes, equally distributing the liquid solvent was critical in creating a smooth, sturdy phosphate binding. This was accomplished by stirring a solution of low concentration sodium phosphate into the calcium phosphate powder rigorously for 45 60 seconds, then placing the wet paste into the palm of the hand, slowly rolling and kneading it into a cylinder. Setting time varied between 8 minutes at $75^{\circ} \mathrm{F}$ and 14 minutes at $65^{\circ} \mathrm{F}$.

\section{g. $\underline{\mathrm{CaP}, 12.0 \mathrm{~mm}}$}

The $12.0 \mathrm{~mm}$ voids were wide enough to hand place the pre-mixed cylinder of cement directly into the allotted space. A spatula (included in each kit) was used to compress the paste so that it filled the entire void to minimize air pockets within the foam block. Screws were then threaded by hand into the center of the filled hole. A spacer was used to verify the screw head was protruding the correct distance out of the cement void. All mixtures were allowed at least 24 hours to cure before testing began.

\section{h. $\underline{\mathrm{CaP}, 4 \mathrm{~mm}}$}


Calcium Phosphate adhesive was observed to frequently trap air within the hole upon insertion; therefore a small 0.08 " $(2 \mathrm{~mm})$ vent hole was drilled into each $4 \mathrm{~mm}$ hole and out the back of the foam block so that air could escape while the cement filled the void evenly. Included in each kit of $\mathrm{CaP}$ was an air tight syringe and plunger that allowed users to easily fill these small perforations. Screws were then inserted by hand and threaded 8-10 turns to verify that the cement was evenly distributed throughout the length of the screw.

\section{Data Acquisition Criteria}

After each screw sample evaluation, the raw data was compiled and essentially condensed into a table of averages and responses to be used later for meaningful tables and charts. This raw data was sometimes difficult to sort through, due to a number of intrinsic characteristics of the test setup. The computer system integrated with the MTS machine was told to record only maximums and minimums during the cyclic testing process to avoid tens of thousands of lines of raw data at the end of each sample. Ideally this would show exactly 8000 data points for a screw sample that survived the full 4000 cycles of testing, with a max and min value for each cycle. However, this was not always the case. The data acquisition program often picked up miniature "wiggle" points throughout the raw data, which added multiple extra peaks and valleys in between the larger, important data points. These extraneous data were easily spotted and avoided 
while gathering averages across different load levels, a step that proved to be quite time consuming in some cases.

The four load levels applied to each screw sample were $\pm 25, \pm 50, \pm 100$ and \pm 200 Newtons as shown above in table I. Another common occurrence that complicated standard data acquisition was screws failing within 2-5 cycles of a new load level. This would often happen when a screw was close to failure at certain load level, but when the 1000 cycles finished and the new, much higher load level began, the screw failed almost immediately. To account for this phenomenon, a general rule was set in place in which to qualify as having adequate data for any given load level a screw must complete at least 50 cycles at that load level. For instance, if a screw failed at cycle 3015 (out of 4000), the Load Strength response (load at time of failure) would still be 200N, but the displacement data for the 15 cycles applied at $\pm 200 \mathrm{~N}$ would be ignored. Instead, the last 10 cycles of the $100 \mathrm{~N}$ range $(2990-3000)$ would be used to calculate displacement. If the proceeding screw sample survived for 3060 samples before failing, Load Strength would again be $200 \mathrm{~N}$ but the final 10 cycles before failure within the $200 \mathrm{~N}$ range (from 3050 to 3060 cycles) would be used for the displacement response. The exception to this rule was when screws failed immediately when testing began (in the $25 \mathrm{~N}$ range). Most of the nonlocking stripped samples in the control group fell within this exception, in which case the displacement was measured as close as possible within the very few cycles recorded.

Another major data complication, although much less common, was the tendency of the MTS to not be able to apply full force to a given sample due to a prolonged failure period. Occasionally, screws would not completely pull out all at once. They might begin to wiggle free, but remain hanging on by a short length of the shank. This anomaly would 
cause much more displacement than screws that pulled directly out all at once. The problem was the MTS has been programmed to cycle at $1 \mathrm{~Hz}$, or as close to that as possible. When a screw suddenly becomes more compliant to a load force, physics demands that more displacement is required to have the same force resistance. The MTS was load dependent during these tests, but it was cycling at a steady $60 \mathrm{~Hz}$ the entire time, which takes precedence over the load endpoints. So in these rare cases when the screw was slowly toggling out of the foam block, the MTS estimates the displacement needed to achieve the goal load force, which might not be enough.

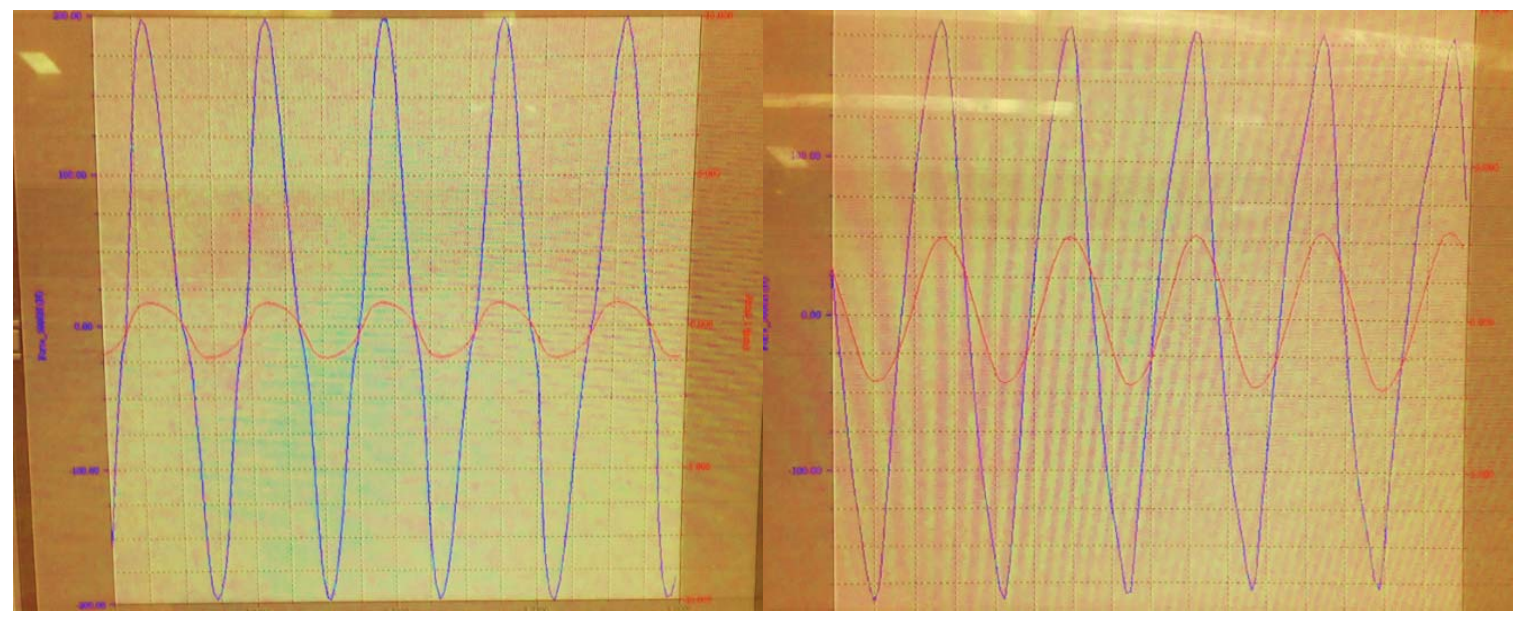

Figure 7: Real time graph of Force and displacement over time for two different samples at $200 \mathrm{~N}$. The blue line represents measured force output from the load cell and the red line is displacement above and below the zero line. Before each test started, the distance sensor was zeroed where the load cell also displayed zero force output. The graph on the left displays a sample that is being cycled at $200 \mathrm{~N}$ and is stable. This sample survived the entire 4000 cycle test. The graph on the right, however, shows an unstable sample being cycled at 200N. Each turn of the MTS forces the screw a little bit further in each direction and the force output was not able to hit the endpoints at $\pm 200 \mathrm{~N}$.

The result of the MTS shortcoming was that the screw displaced much farther in both directions, and continued to slowly wiggle free from the block which then caused even more displacement in each direction. While this was happening, the MTS programming attempted to satisfy the cycle rate parameter as well as the load parameter, 
but the cycle rate took precedence in this case. The resulting raw data then showed that the $1 \mathrm{~Hz}(60$ cycles/min) rate was still intact, but the last $\mathrm{X}$ amount of cycles while the screw was "walking" out of the block exhibited significantly reduced load level endpoints. To account for this, another rule had to be made: Displacement data cannot be used for sample averages unless the load level at that data point was within $10 \%$ of the desired force. After all, if the screw has begun walking out of the foam block enough to continue to creep at every half cycle it has essentially failed to keep any sort of plate secure to the bone in a practical sense. The most common instance of this was in the last load level of $200 \mathrm{~N}$, in which the rule demands that the displacement averages can only start where a load of $\pm 180 \mathrm{~N}$ or more was recorded.

\section{CaP "test" and "retest" samples}

Stainless steel $4 \mathrm{~mm}$ cancellous bone screws were used throughout the entirety of this study, but towards the end of the PMMA trials, the supply of screws ran out and it would be another few months before more were shipped to the lab. Due to the lack of screws around the beginning of the calcium phosphate trials, a new screw insertion method was implemented and tested for the $12.0 \mathrm{~mm}$ hole low density locking and nonlocking samples. The calcium based cement was mixed normally and manually inserted into the low density blocks. Once the cement hardened a similar approach to the Plaster of Paris trials described above was executed, with some samples inserted by hand and others inserted with a drill. However, the "hand" threaded samples in this case were 
different than the hand threaded Plaster of Paris trials. Because there were no extra screws present to immediately thread into the wet cement shortly after mixing, the cement was allowed to cure for a few minutes before a single screw was carefully threaded into and then back out of each "hand" cement sample. The resulting indents left within the cement were the same size and thread pitch of the cancellous screws.

When more screws finally arrived they could be carefully inserted into the premade casts to resemble the hand threaded technique. This alternate technique was referred to as "modified hand" threaded. The remaining "TEST" samples in this set were allowed to cure completely, and then an inner diameter sized hole was drilled out from the center of the cement in accordance with the "drill" technique described above. A total of 12 "modified hand" samples (6 locking and 6 nonlocking) and 8 "drill” samples (4 locking and 4 nonlocking) were used due to the nature of the foam blocks that were available at the time.

After these 20 total screws were tested, the data was analyzed to determine if the "modified hand" technique was statistically similar to the "drilled" samples. The hypothesis in this miniature experiment within an experiment was that if the modified hand technique was administered correctly, there should be no statistical difference between the two methods, although the sample size was relatively small. This hypothesis stems from the results in the Plaster of Paris pretrials described above, where the standard "hand" threaded method and "drill" inserted method were calculated as not significantly different. Also, the calcium phosphate based cement was very similar in composition to the Plaster of Paris. 

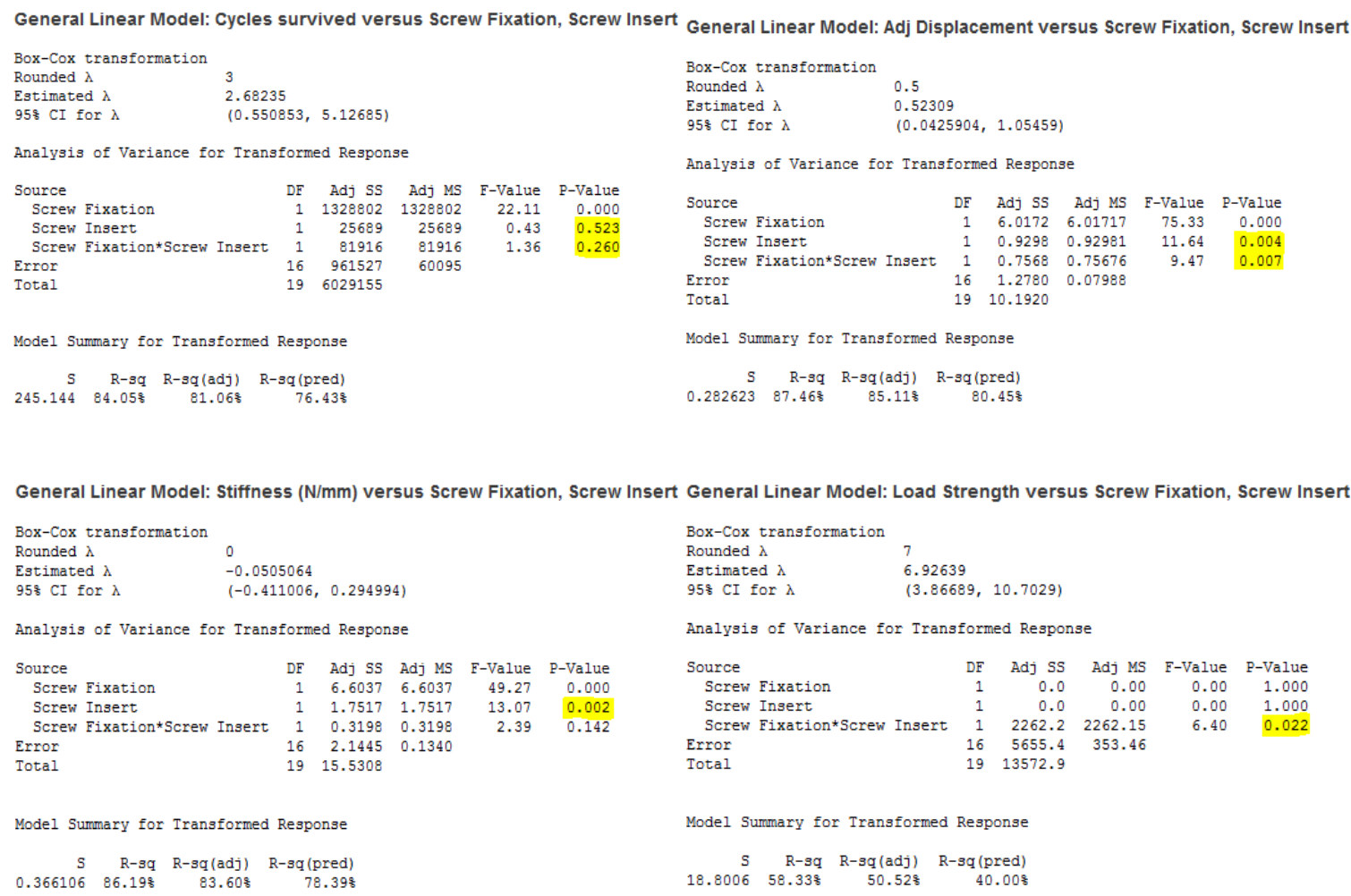

Figure 8: General linear model ANOVA results for the "modified hand" vs "drill" comparison in the low density calcium phosphate trials. All four major responses in this study were considered: Cycles Completed (top left) Adjusted Displacement (top right) Stiffness (bottom left) and Load Strength (bottom right). The two factors used for this comparison were Screw Fixation (locked or nonlocked) and Screw Insert (modified hand or drill) as well as the interaction between the two. A Box-Cox transformation was applied to all four models, with Lambda values shown for each. Relevant P-values are highlighted for each response.

Other than the Cycles Completed response (above, top left) all other responses

showed either the Screw Insert factor or the factor interaction as significant. Most importantly, though, was the adjusted displacement response for which both the Screw Insert factor and the Screw Fixation*Screw Insert factor interaction displayed P-values of 0.004 and 0.005 respectively. Because the Screw Insert factor was statistically significant, it means there was a difference between the "modified hand" technique and the "drill" technique. This proved that the "modified hand" insertion method was NOT administered correctly and should be redone. 
Twelve "modified hand" inserted screws were re-tested, while the 8 "drill" inserted screws were left alone. More than enough screws were available this time, so the "modified hand" technique wasn't necessary. Calcium phosphate cement was mixed as usual and screws were inserted within minutes into the curing cement in accordance with the standard "hand" insertion technique described in the Plaster of Paris section above. Also, for each fixation factor, locking and nonlocking, 5 screws were inserted by hand and 1 screw was inserted later via the drill so as to make both groups of low density large hole CaP a total of 5 "hand" threaded and 5 "drill" inserted samples. The 12 samples that were redone were named "RETEST CaP..." while the 8 samples that were drilled originally kept the name "TEST CaP..."

\begin{tabular}{|l|}
\hline RETEST Large Low Locking 1 \\
\hline RETEST Large Low Locking 2 \\
\hline RETEST Large Low Locking 3 \\
\hline RETEST Large Low Locking 4 \\
\hline RETEST Large Low Locking 5 \\
\hline RETEST Large Low Locking 6 \\
\hline TEST Large Low Locking 7 \\
\hline TEST Large Low Locking 8 \\
\hline TEST Large Low Locking 9 \\
\hline TEST Large Low Locking 10 \\
\hline RETEST Large Low Nonlocking 1 \\
\hline RETEST Large Low Nonlocking 2 \\
\hline RETEST Large Low Nonlocking 3 \\
\hline RETEST Large Low Nonlocking 4 \\
\hline RETEST Large Low Nonlocking 5 \\
\hline RETEST Large Low Nonlocking 6 \\
\hline TEST Large Low Nonlocking 7 \\
\hline TEST Large Low Nonlocking 8 \\
\hline TEST Large Low Nonlocking 9 \\
\hline TEST Large Low Nonlocking 10 \\
\hline
\end{tabular}

Figure 9: Low Density $12 \mathrm{~mm}$ (large) hole Calcium phosphate samples. All 20 samples were originally named "TEST" to signify that the first 6 screws of both locking groups were inserted via an alternate "modified hand" technique while the remaining 4 screws were inserted via the standard "drill" technique described above. After testing for statistical differences, the modified hand insertion method was proven inadequate, so those 12 samples were redone and named "RETEST." Upon retesting, the first 5 screws were inserted by the standard "hand" method (also described above, Plaster of Paris) while the $6^{\text {th }}$ screw followed standard "drill" protocol. The dotted line above denotes the split between "hand" and "drill" insertion methods. 
The same statistics were calculated for the 20 screw samples displayed above in

Figure 9 for the RETEST samples. These results are as follows:
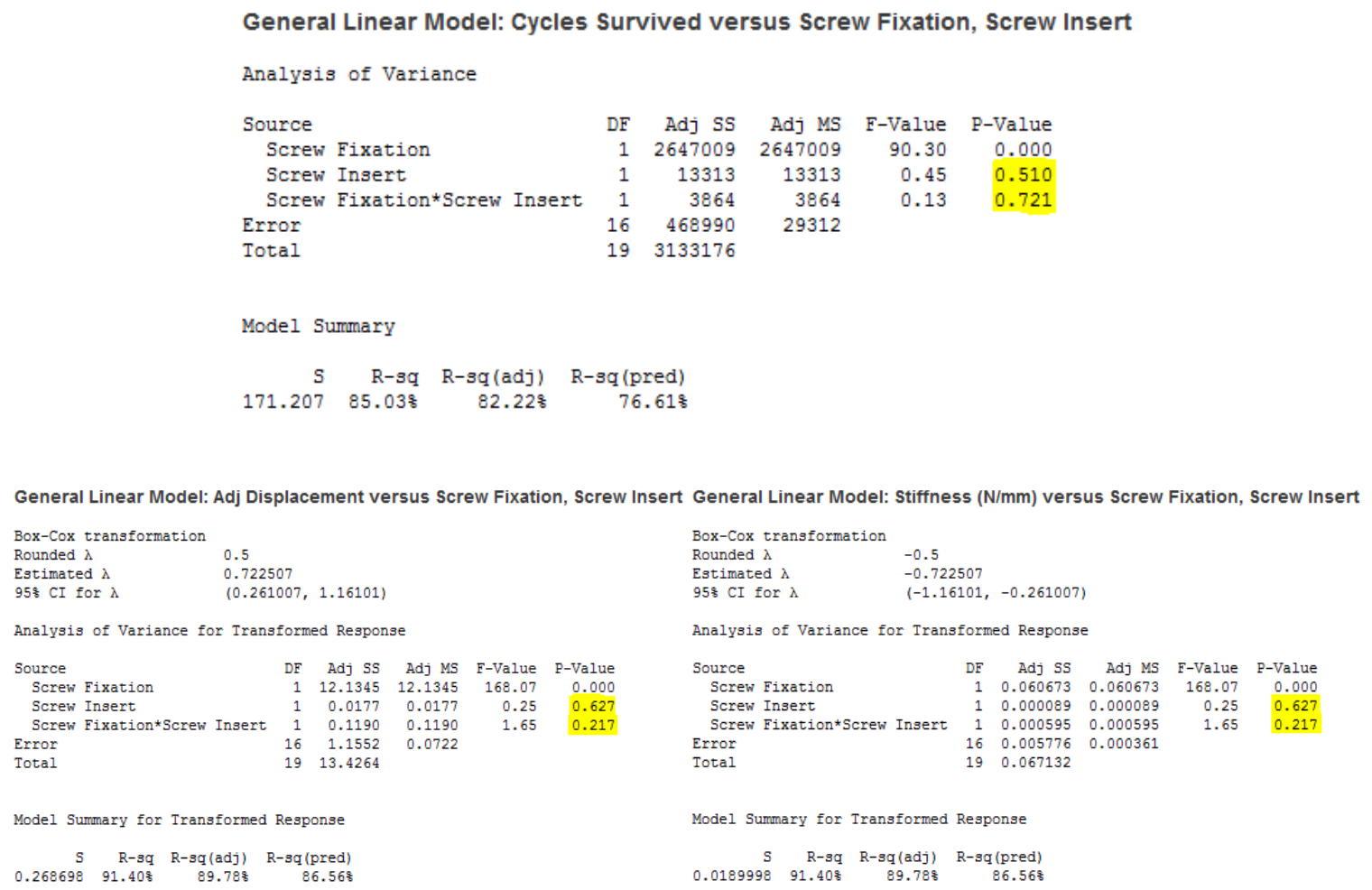

Figure 10: General linear model ANOVA results for Cycles Survived (top) Adjusted Displacement (bottom left) and Stiffness (bottom right) for Hand vs Drill RETEST of low density $12 \mathrm{~mm}$ hole Calcium phosphate samples. The Screw Insert factor used in this model refers to screws either being hand threaded or inserted via a drilled hole, as described above in the Plaster of Paris section. Load Strength was ignored for this test because all 20 samples recorded a $200 \mathrm{~N}$ load level. A Box-cox transformation was assumed as necessary for all three responses, but the transformation coefficient of the Cycles Survived response (top) was $\lambda=-1$, so the ANOVA was run again without a transformation. Relevant P-values are highlighted for convenience.

The P-values for the different responses in the RETEST model shown above in

Figure 10 indicate that this time around there was no significant statistical difference

between the standard "hand" threaded and "drill" insertion method. Save the Load

Strength response (all samples recorded a 200N final load level) all responses exhibited a

P-value greater than 0.200 for the Screw Insert factor and the Screw Fixation*Screw 
Insert factor interaction. This proved once again that the hand and drill insertion methods are both adequate screw insertion techniques. This also showed that the "modified hand" technique from the first round TEST samples was NOT an acceptable insertion technique.

\section{E. Failure mode under locking plate}

Screw samples failed in this cyclic load experiment in two ways; either the screw wiggled free from the foam (and/or surrounding cement) and pulled completely out of the block or the constant cyclic pressure caused the screw to bend somewhere along the thread shaft and eventually break. The majority of "pullouts" occurred when the locking plate was attached while most "breakages" resulted from the nonlocking custom plate. The locking plate kept the screw rigid within the foam block which allowed for very little "wiggle" room and the nonlocking plate let the screw toggle freely, exposing the body of the screw to bending forces often resulting in screw breakages. These failure modes raise an interesting point regarding the use of standard screw types for both locking and nonlocking plate constructs.

Typical cancellous bone screws or any bone screws for that matter with a locking plate head (narrow threads on the head of the screw) are designed differently than their non-locking counterparts. Locking head screws have a cone shaped head with threads top to bottom to seal (or "lock") the screw inside the locking plate at a 90 degree angle. Along with this abnormal head shape, the body of locking head screws is substantially thicker than traditional screws, particularly in the "neck" of the screw just under the head. 


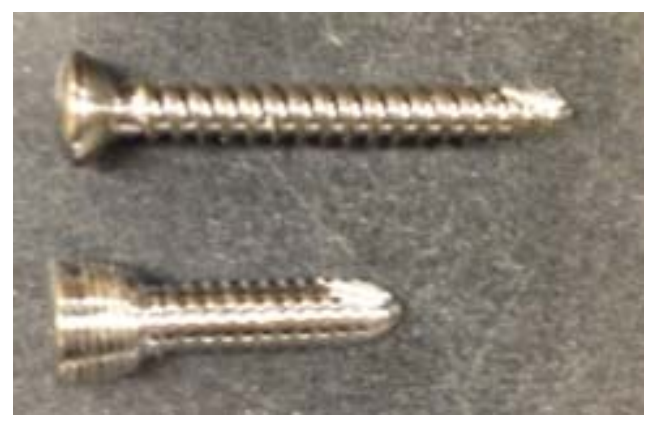

Figure 11: A visualization of the difference between locking head screws (bottom) and traditional smooth head screws (top). The locking head screws have threads along the head which is thicker than the alternative, and it transitions directly to the body without a "neck" region. This design increases the strength throughout the locking screw and allows the plate to hold it in a "locked" 90 degree angle.

In an effort to normalize screw samples across the entire study, only traditional "non-locking" screws were used for testing along with the custom attachment plate that simulated the rigid locking head or flexible nonlocking construct. Since conventional locking head screws are naturally thicker in the body and neck area they would inherently be stiffer than smooth head screws regardless of plate attachment. Using the traditional smooth head screws for both locking and nonlocking testing samples creates a unique problem. There's a good reason locking head screws have a much thicker body and neck circumference: when the screw is held static in a perpendicular angle and the surrounding plate is subjected to outside forces, most of the stress is focused just behind the head of the screw, directly on the neck. The custom locking attachment plate successfully holds these screws at a rigid 90 degree angle during testing to simulate a locking plate, but it still uses traditional smooth head screws with a skinny neck. If a large amount of samples were to fail via screw breakages at the neck area of the screw while the locking custom plate was attached the argument could be made that this setup is not a valid system.

Fortunately, of the 76 total recorded breakages in locking samples, only three total samples actually broke just behind the screw head at the neck. The remaining 73 screws 
broke between the first and $5^{\text {th }}$ thread down the shaft. This was most likely due to the novel counter weight pulley system utilized throughout the experiment. As the screws were cycled up and down, the counter weight constantly provided a normal pullout pressure which forced the focal point of the cyclic loading from the neck of the screw downward into the threaded body. Breakages within nonlocking screw samples appeared in the same area of the screw body which validates this technique. The three neck breakage screw samples were recorded with an asterisk in the failure mode column in the Raw Data section. This subject is discussed further in the Discussion section at the end of the paper.

\section{F. Inherent Displacement System Error}

The setup used for this study was made by hand and therefore has inherent flaws within the system. Finite movement was the base response for the entire project and any extra movement within the system can significantly blur the results. Therefore it was very important to quantify the inherent movement error within the system, otherwise known as the "slop displacement." The slop displacement values were calculated by inserting a screw into a hard plastic block and running the same cyclic test at 50 cycles per load level. The hard plastic kept the screw static, so the displacement measured during this test was only the inherent movement within the testing rig itself. These values are listed as follows: 
Table II: Slop Displacement Values per Load Level

\begin{tabular}{|c|c|}
\hline \multicolumn{2}{|c|}{ Slop Displacement (mm) } \\
\hline Load Level & Displacement \\
\hline 25 & 0.0460 \\
\hline 50 & 0.1021 \\
\hline 100 & 0.2248 \\
\hline 200 & 0.5226 \\
\hline
\end{tabular}

After these error values were calculated, they were subsequently subtracted from every measured displacement value throughout the study. The resulting values were denoted as "adjusted displacement" to signify that the inherent error had been accounted for. The same hard plastic block used to calculate the slop displacement was also used for the theoretical screw bending validation section below.

\section{G. Theoretical Screw Bending Validation}

This section will take a look at the fundamental math behind the behavior of the screws in this study. When an MTS machine was cyclically loading the installed screws, each individual push or pull displaced the head of the screw by a finite amount as a function of the force exerted. In this case, the screw can be considered a simple cylindrical beam subjected to a lateral load over multiple cycles. A model can be created for this movement using beam theory. The two cases for this model will fall under the locking and nonlocking categories. Starting with the more basic model, the nonlocking interface behaved like a single fixed end bending beam, shown below. 


\begin{tabular}{|c|c|c|c|}
\hline $\begin{array}{l}L=\text { overall length } \\
W=\text { point load, } M=\text { moment } \\
w=\text { load per unit length }\end{array}$ & End Slope & Max Deflection & $\begin{array}{c}\text { Max bending } \\
\text { moment }\end{array}$ \\
\hline & $\frac{W L^{2}}{2 E I}$ & $\frac{W L^{3}}{3 E I}$ & $W L$ \\
\hline
\end{tabular}

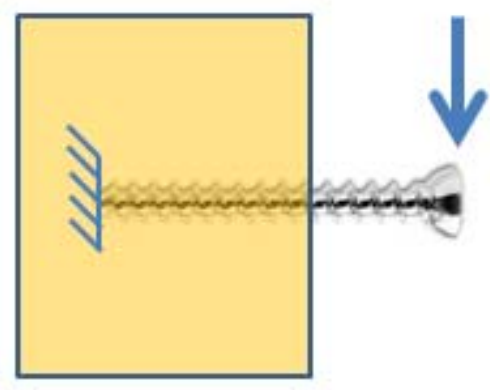

Figure 12: Beam bending theory values, single fixed end (top). This model translates to the nonlocking custom plate, which is modeled in the sketch (bottom). The block held the base of the screw rigid while the MTS cycled the head of the screw, which was free to toggle. Image taken from Beam Deflections, Second Order Model [28].

Because the nonlocking attachment of the custom plate doesn't grip the head of the screw, the long end penetrating the polyurethane foam block was the only fixed or non-moving end, illustrated in Figure 12 above. The nonlocking attachment let the head toggle freely within the plate hole (custom plate slot). The distance the screw head moved each time was the displacement $\delta$, which was equal to the Max Deflection equation in figure 12 above. W corresponds to the point load of $25,50,100$, or $200 \mathrm{~N}$. L is the overall length of the beam, a dimension that does not necessarily mean the full length of the screw.

In the majority of cases in this study, either the surrounding cement or the foam block itself was gripping the screw the entire length that was not exposed. This means that the edge of the fixed end was wherever the screw was securely fastened within the block. Correspondingly, as the testing progressed through its cycles, the fixed location 
may change. As the screw toggled up and down by increasing loads, this naturally wore down the polyurethane and/or cement closest to the site of displacement. It may be an entirely new project on its own to quantify the rate of degradation within the cement immediately surrounding the screws and coinciding length L, but for this study it was assumed that the overall length L was fixed for each sample.

Other assumptions can be made about the EI value. E is the Young's Modulus, or Modulus of Elasticity, of the material which in this case was stainless steel. According to [27] stainless steel has a modulus of roughly $180 \mathrm{GPa}$, or $180 \times 10^{9} \mathrm{~N} / \mathrm{m}^{2}$. The "I" value is the moment of inertia of the shape of the material, observing from the normal axis, in $\mathrm{mm}^{4}$. The body of a screw, neglecting the screw threads, is similar to a simple cylinder with an inner diameter of about $2 \mathrm{~mm}$.

A more advanced model is needed to replicate the nature of the locking attachment plate. In this case, the locking plate firmly secured the head of the screw in place so that no immediate deflection occurred. It was essentially another fixed end, with the main deflection taking place in the body of the screw. See Figure 13 below: 


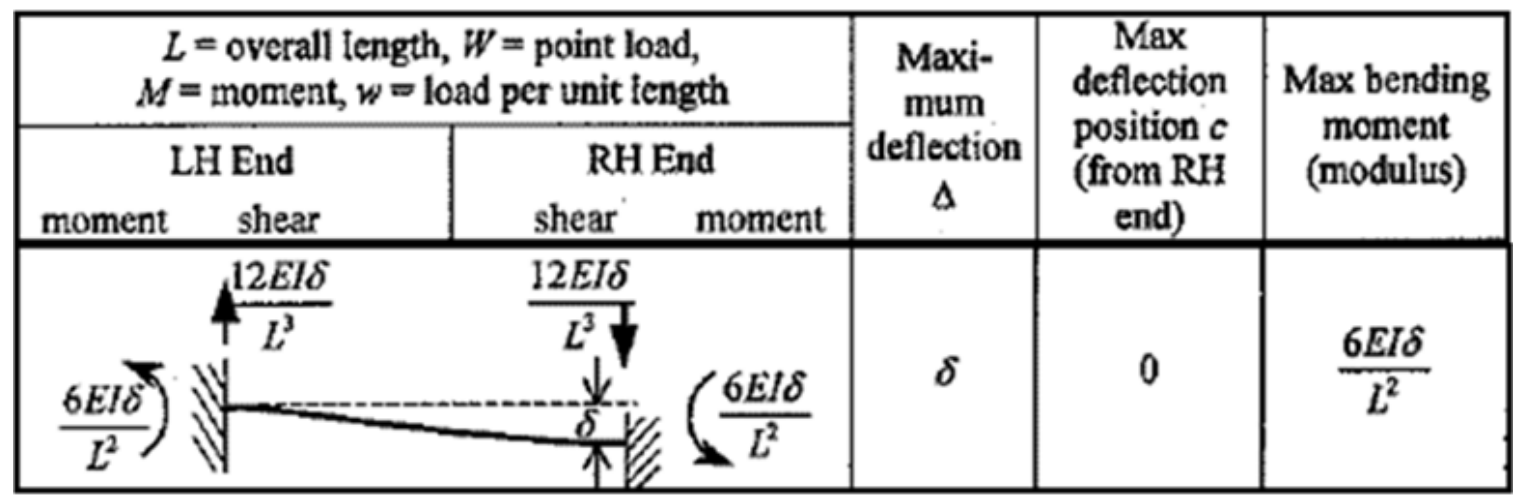

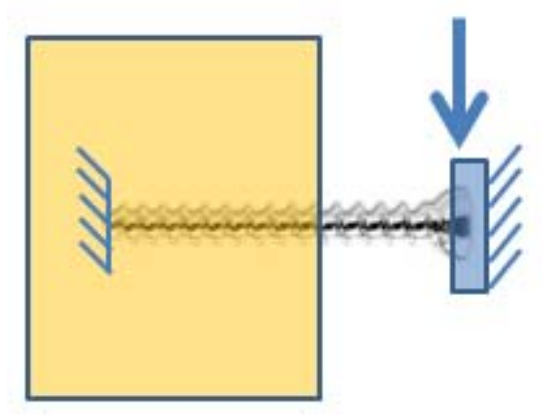

Figure 13: Beam bending theory values, double fixed end (top). This model more closely resembles the locking head custom plate for this study, which is modeled below. The block held the tail end of the screw rigid and the locking plate attached to the custom plate also held the head of the screw rigid while the MTS machine toggled the plate vertically. Image taken from Beam Deflections, Second Order Model [28].

The shear end load still affected the beam at the same place, but the loaded end was also fixed to form a right angle at all times. Solving for the deflection $\delta$ resulted in a deflection of $\mathrm{WL}^{3} / 12 \mathrm{EI}$. Comparing this deflection value to that of the simpler model with the nonlocking attachment, $\delta_{\text {nonlocking }}=4 \delta_{\text {locking. }}$. So in theory, under the same end load, with the same length and type of screw, it is expected that a sample using the nonlocking plate would experience four times the displacement as the same sample using the locking plate.

To evaluate this theory, a simple test protocol was applied to a screw fastened into a static hard plastic block at a known distance. 50 cycles at $25 \mathrm{~N}$ were applied to the head 
of a screw that was protruding $10 \mathrm{~mm}$ out from the block. This was repeated with both locking and nonlocking attachment plates, as well as with and without the counterweight. Data was collected several times every millisecond rather than at minimums and maximums to better portray the shape of the displacement per load curve. The final displacement data is shown in Table III below. For a better visual of the displacement per load curves for all Screw Bending Theory samples, reference Figure 14 below.

Table III: Screw Bending Theory Displacement Results (mm)

\begin{tabular}{|l|c|c|}
\hline & Locking & Nonlocking \\
\hline Weight & 0.286 & 0.648 \\
\hline No Weight & 0.251 & 0.872 \\
\hline
\end{tabular}

The first take away was the noticeable difference between locking and nonlocking displacements, as predicted by the screw bending theory. Comparing the samples that did not use the counterweight, the ratio between the locking and nonlocking attachments was $(0.871558) /(0.251477)=>3.466: 1$, which is fairly close to the theoretical ratio of $4: 1$. The ratio for the set that did use the counterweight was slightly less at 2.27:1, and the average of the two sets was 2.83:1. These results differ from the calculated ratio due to a number of reasons, with the main variable being the geometric shape of the screws. The inner diameter was a nice even cylinder, but addition of the threads on the sides will influence the specific moment of inertia to some degree.

Testing theoretical displacements not only revealed ratios between locking and nonlocking attachments, it also allowed for the calculation of multiple otherwise unknown variables in these tests and regular test samples elsewhere. With a known length 
$\mathrm{L}$ and force $\mathrm{W}$, a more accurate EI value can be calculated for known displacements. The example below used the data from Nonlocking with Weight 1.

$$
\begin{gathered}
2 \delta=0.644195 \mathrm{~mm} \text {, or } 0.0006442 \mathrm{~m} \\
\rightarrow \delta=0.000322 \mathrm{~m}, L=10 \mathrm{~mm}=0.01 \mathrm{~m}, \mathrm{~W}=25 \mathrm{~N}
\end{gathered}
$$

Line (1) lists the known variables, starting with the displacement. The original value given is the result of $\pm 25 \mathrm{~N}$, and $\delta$ is the displacement from the center line so it was halved and converted to meters.

$$
\delta=\frac{W L^{3}}{3 E I} \rightarrow 0.000322 m=\frac{(25 N)(0.01 m)^{3}}{3 E I}
$$

After converting all the variables, they were plugged into the equation for a single fixed end as this was nonlocking data.

$$
0.000322 m=\frac{0.000008333 N * m^{3}}{E I}
$$


Simplifying in line (3), it was important to keep the units consistent. The EI value should be in $\mathrm{Nm}^{2}$.

$$
E I=\frac{0.000008333 N * m^{3}}{0.000322 \mathrm{~m}}=0.0259 \mathrm{~N} * \mathrm{~m}^{2}
$$

Solving for EI, line (4) showed that $\mathrm{EI}=0.025872 \mathrm{Nm}^{2}$. Estimating an EI value from the Young's modulus of stainless steel and the moment of inertia of a cylindrical beam gives a value of $\mathrm{EI}=0.13613 \mathrm{Nm}^{2}$. This may look very inaccurate, but it is only a difference of using $1.2 \mathrm{~mm}$ as the diameter rather than $2 \mathrm{~mm}$. The diameter was raised to the $4^{\text {th }}$ power to calculate moment of inertia, so even tiny changes result in large differences, and the inner diameter of a screw with threads throughout its length was difficult to estimate.

The same process shown above to calculate an EI value was repeated for all screw bending theory tests to arrive at an average EI of $0.019408 \mathrm{Nm}^{2}$. 


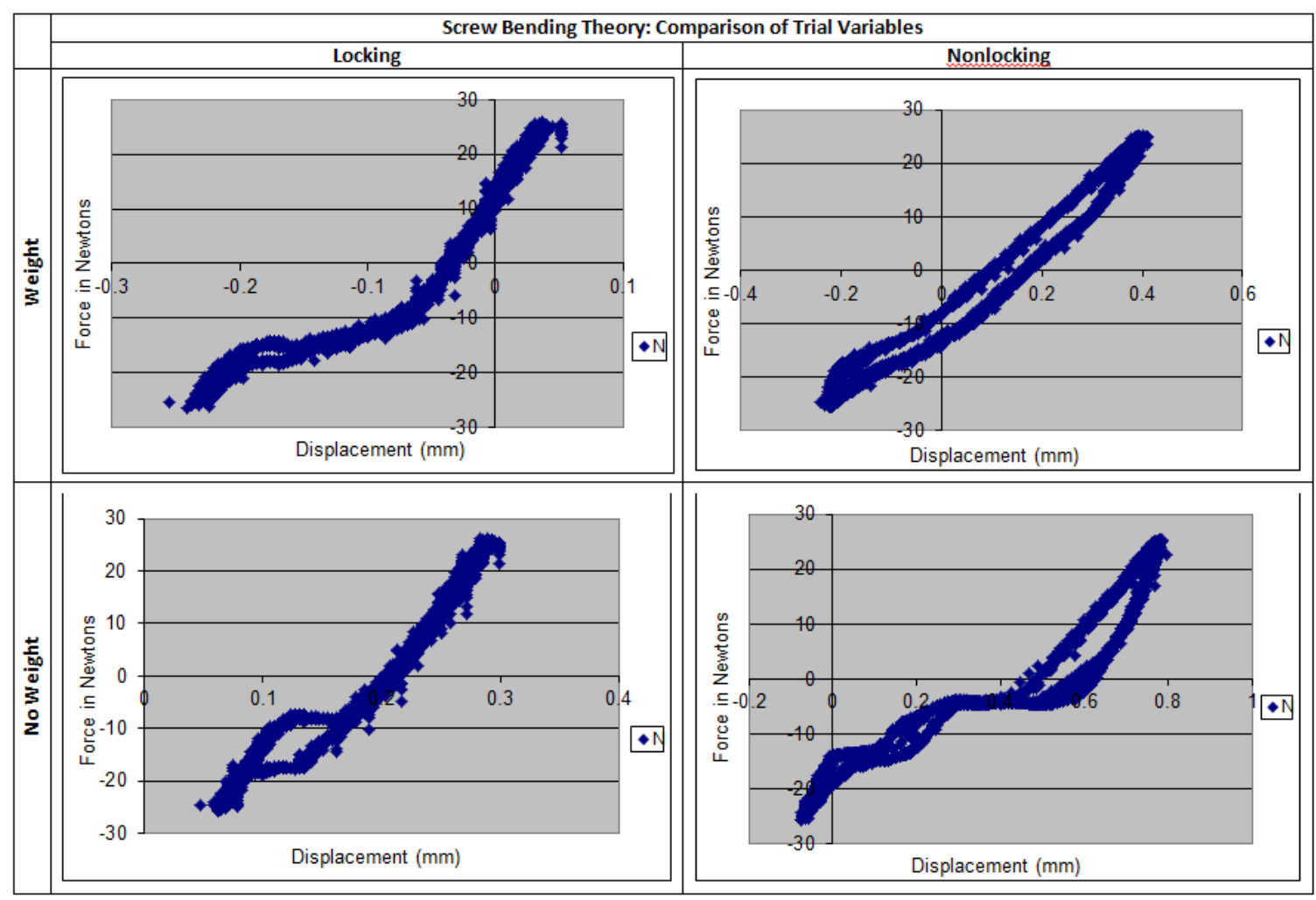

Figure 14: A visual comparison of the four different force displacement curves for combinations of Locking, nonlocking, counterweight and no counterweight screw bending theory trials. Hysteresis is apparent in all but the Locking with weight sample. The largest displacement was shown in the Nonlocking no weight trial. 


\section{STATISTICAL ANALYSIS}

Once all the data had been collected and entered into the appropriate tables, a statistical analysis was necessary to find an accurate model for the data. Also, inherent outliers will naturally occur with a large data set and the analysis will show which data can be safely omitted from the final results. Before calculating the statistical outliers, a few samples will be dropped due to machine or human error during testing:

- Control Stripped Low Locking 10 - Computer error; failed to record data

- PMMA Large Low Locking 4 - Human error; debris inside hole caused inadequate cement adhesion, cement and screw pulled out of foam block

- CaP Stripped Low Nonlocking 1 - Machine error; manual restart caused MTS hydraulics to ascend and twist, destroying screw before testing began

- CaP Stripped High Nonlocking 6 - Human error; not enough cement inserted into the space; inadequate cement / block interdigitation.

The four samples listed above were manually removed from the full data set. The data in this project were constrained by four factors and four responses, as follows. 
Factors:

- Cement Type

- Hole Size

- Bone (Foam) Density

- Screw Fixation

Responses:

- Cycles Survived

- Adjusted Displacement (mm)

- Load Strength (load level at failure, in Newtons)

- Stiffness (load level over Displacement, N/mm)

To find an acceptable model for the data, all significant factors and factor interactions had to be taken into account for each individual response. Cycles Survived was first.

\section{A. Cycles Survived}

A general linear model ANOVA was selected as the most appropriate statistical approach to this data. First, the ANOVA was calculated with all factors and factor interactions through the third order. 


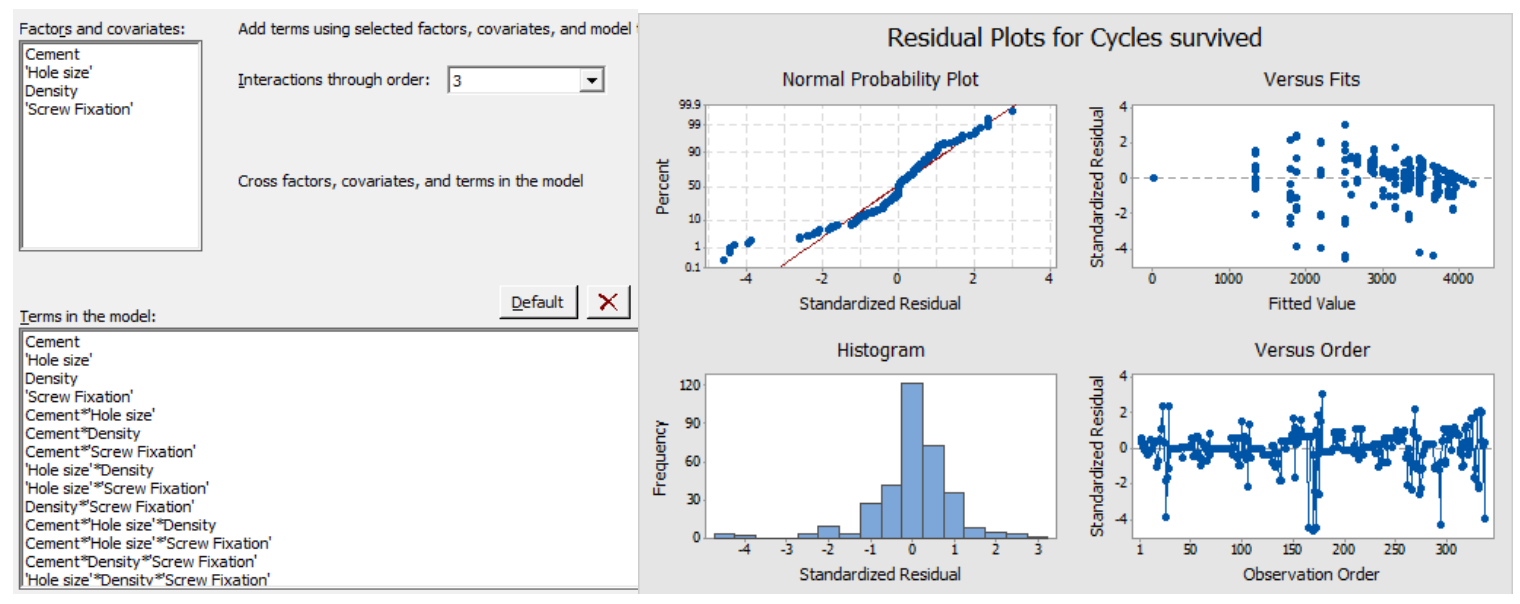

Figure 15: Factor interactions (left) and standardized residuals (right) for general linear model ANOVA, cycles survived.

Upon observation of the residual plots shown above, one can infer that a data transformation was necessary for this response. The Normal Probability Plot wasn't linear and the Versus Fits data layout was cone shaped. A box cox transformation was needed.

The one-parameter Box-Cox transformations are defined as:

$$
y_{i}^{(\lambda)}= \begin{cases}\frac{y_{i}^{\lambda}-1}{\lambda} & \text { if } \lambda \neq 0, \\ \ln \left(y_{i}\right) & \text { if } \lambda=0\end{cases}
$$

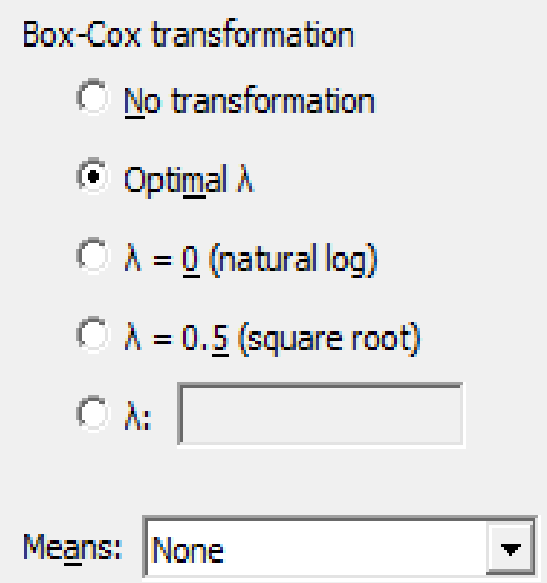

Box-Cox transformation
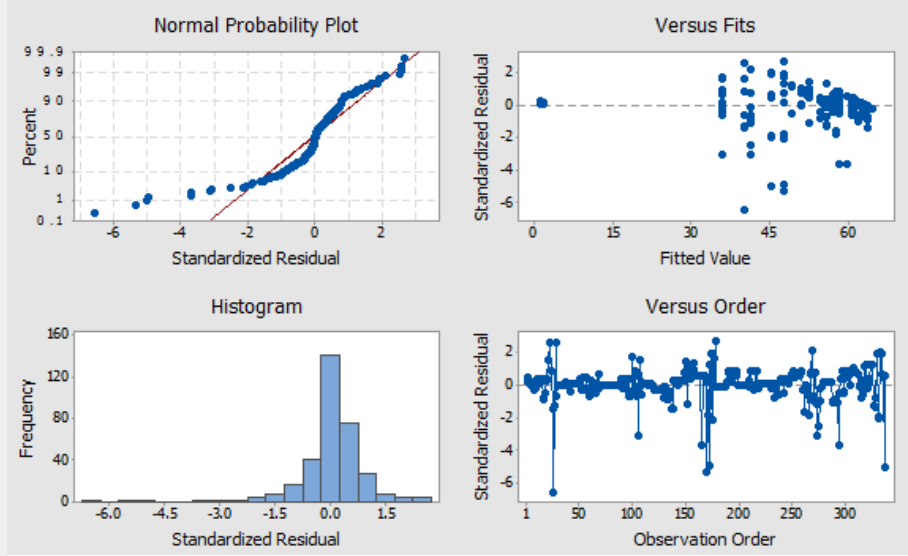
Figure 16: Optimal Box-Cox transformation (bottom left) and resulting standardized residuals (bottom right), general linear model ANOVA, cycles survived. The standard piece-wise function for a single parameter Box-Cox transformation is shown at the top.

Unfortunately these transformed results didn’t appear much better, but the adjusted R-squared value went from $83.9 \%$ to $89.03 \%$ after the transformation $(\lambda=0.5)$ so it was still useful. The Lambda value of 0.5 also shows that the transformation wasn't very powerful. After all, real data is hardly ever perfect. Luckily, 3 interactions could not be estimated by Minitab and were excluded (Cement*Hole size, Cement*hole size*density, Cement*hole size* screw fixation) and the rest of the interactions were significant at a $90 \%$ confidence level $(\alpha=0.1)$.

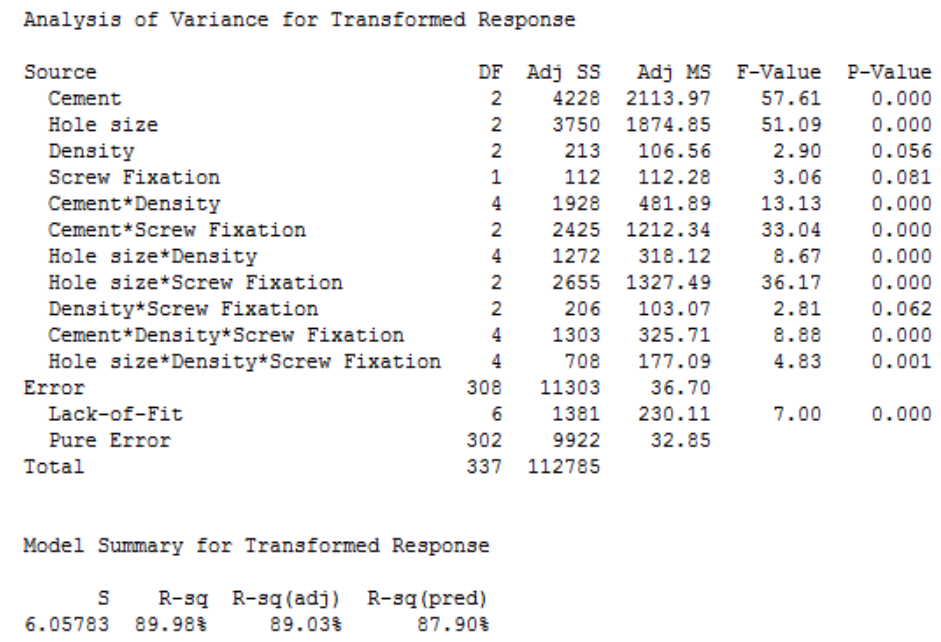

Figure 17: Significant factor interactions and subsequent P-values for the cycles survived response.

Now that an applicable model has been determined for this response, unusual observations (outliers) can be documented. These will be compared at the end with the unusual observations from the other responses to determine which samples can be considered as true outliers and omitted from the data set. Minitab lists all samples with a residual of \pm 2.0 but standard practice often denotes a residual of \pm 3.5 as being an outlier, 
so anything above or below 3.0 will be deemed notable and \pm 3.5 will be listed as statistically distinct for this analysis.

Table IV: Notable Outliers from Cycles Survived Response

\begin{tabular}{|c|c|c|}
\hline Observation number & Sample Name & Standardized Residual \\
\hline 26 & Control Stripped Low Locking 6 & -6.57 \\
\hline 106 & Control Stripped High Locking 7 & -3.12 \\
\hline 165 & PMMA Stripped Medium Locking 7 & -3.70 \\
\hline 170 & PMMA Stripped Medium Nonlocking 2 & -5.34 \\
\hline 173 & PMMA Stripped Medium Nonlocking 5 & -4.96 \\
\hline 274 & CaP Stripped Low Nonlocking 7 & -3.09 \\
\hline 294 & CaP Stripped Medium Locking 1 & -3.68 \\
\hline 337 & CaP Stripped High Nonlocking 9 & -5.04 \\
\hline
\end{tabular}

\section{B. Adjusted Displacement}

A general linear model ANOVA was run with the adjusted displacement response. All factors and factor interactions through the $3^{\text {rd }}$ order were included.

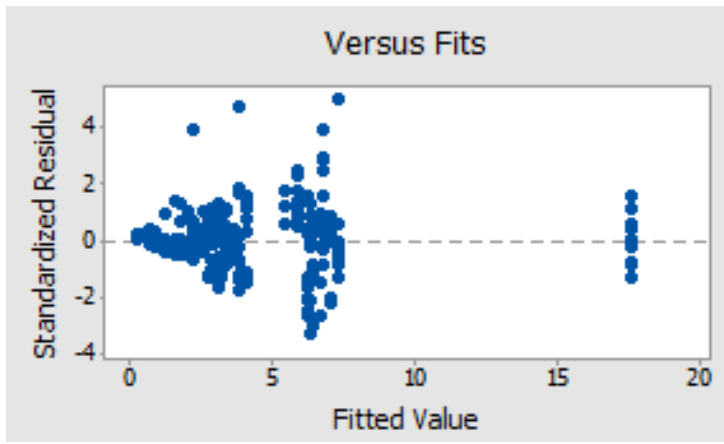

Figure 18: Visualization of standard residuals and fitted values for adjusted displacement general linear ANOVA.

The adjusted R-squared value for this model was $77.51 \%$. Due to the uneven distribution of residuals and left-to-right cone shape in the fitted value plot in Figure 18 above, a box-cox transformation was needed to attain a more accurate model. 


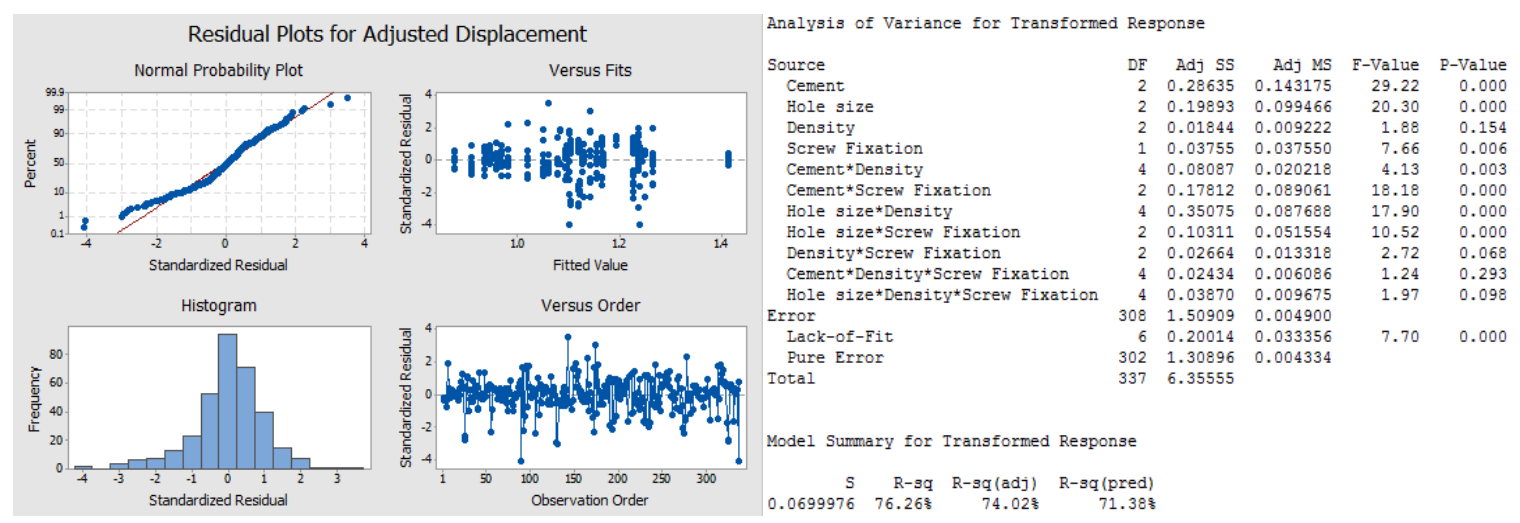

Figure 19: Residual plots after an optimal $\lambda$ box-cox transformation (left) and corresponding Pvalues (right) from the general linear model ANOVA for adjusted displacement.

The new results appeared much cleaner than before, as the fitted value plot was evenly spread out and not cone shaped. The adjusted R-squared value was $74.02 \%$, which is slightly less than $77.51 \%$ before the transformation. Like the ANOVA for cycles survived above, Minitab automatically excluded Cement*Hole size, Cement*hole size*density, Cement*hole size* ${ }^{*}$ screw fixation interactions. The P-value of the Cement*Density*Screw fixation three way interaction was 0.293 , so it can be removed from the model. Rerunning the ANOVA without this interaction yields the following:

$\begin{array}{lrrrrr}\text { Analysis of Variance for Transformed Response } & & \\ & \text { DF } & \text { Adj SS } & \text { Adj MS } & \text { F-Value } & \text { P-Value } \\ \text { Source } & 2 & 0.31710 & 0.158552 & 43.04 & 0.000 \\ \quad \text { Cement } & 2 & 0.17853 & 0.089263 & 24.23 & 0.000 \\ \text { Hole size } & 2 & 0.00730 & 0.003651 & 0.99 & 0.372 \\ \text { Density } & 1 & 0.06396 & 0.063963 & 17.36 & 0.000 \\ \text { Screw Fixation } & 4 & 0.19028 & 0.047569 & 12.91 & 0.000 \\ \text { Cement*Density } & 2 & 0.44470 & 0.222349 & 60.36 & 0.000 \\ \text { Cement*Screw Fixation } & 4 & 0.37501 & 0.093751 & 25.45 & 0.000 \\ \text { Hole size*Density } & 2 & 0.12041 & 0.060204 & 16.34 & 0.000 \\ \text { Hole size*Screw Fixation } & 2 & 0.01405 & 0.007026 & 1.91 & 0.150 \\ \text { Density*Screw Fixation } & 4 & 0.03034 & 0.007585 & 2.06 & 0.086 \\ \text { Hole size*Density*Screw Fixation } & 312 & 1.14938 & 0.003684 & & \\ \text { Error } & 10 & 0.16609 & 0.016609 & 5.10 & 0.000 \\ \quad \text { Lack-of-Fit } & 302 & 0.98329 & 0.003256 & & \\ \quad \text { Pure Error } & 337 & 4.76376 & & & \\ \text { Total } & & & & \end{array}$

Model Summary for Transformed Response

$\begin{array}{rrrr}\text { S } & \text { R-sq } & \text { R-sq(adj) } & \text { R-sq (pred) } \\ 0.0606952 & 75.878 & 73.94 \% & 71.66 \%\end{array}$

Figure 20: ANOVA results and P-values after removing insignificant three way interaction, adjusted displacement. 
The adjusted R-squared value was still roughly the same at $73.94 \%$. Density had a P-value of 0.372 , which was more than enough to confidently remove the factor and rerun the ANOVA. Note: removing one of the main four factors seems counter intuitive and causes the model to no longer be hierarchical, however, several interactions involving density (Cement*Density and Hole size*Density for example) still remain as significant factors. The Density*Screw fixation interaction can also be removed.

\begin{tabular}{|c|c|c|c|c|c|}
\hline Source & $\mathrm{DF}$ & Adj SS & Adj MS & F-Value & P-Value \\
\hline Cement & 2 & 34.248 & 17.1238 & 62.23 & 0.000 \\
\hline Hole size & 2 & 17.039 & 8.5196 & 30.96 & 0.000 \\
\hline Screw Fixation & 1 & 27.639 & 27.6394 & 100.44 & 0.000 \\
\hline Cement*Density & 4 & 19.250 & 4.8126 & 17.49 & 0.000 \\
\hline Cement*Screw Fixation & 2 & 33.458 & 16.7291 & 60.79 & 0.000 \\
\hline Hole size*Density & 4 & 29.093 & 7.2732 & 26.43 & 0.000 \\
\hline Hole size*Screw Fixation & 2 & 8.931 & 4.4657 & 16.23 & 0.000 \\
\hline Hole size*Density*Screw Fixation & 4 & 2.470 & 0.6176 & 2.24 & 0.064 \\
\hline Error & 316 & 86.955 & 0.2752 & & \\
\hline Lack-of-Fit & 14 & 12.417 & 0.8869 & 3.59 & 0.000 \\
\hline Pure Error & 302 & 74.538 & 0.2468 & & \\
\hline Total & 337 & 356.079 & & & \\
\hline \multicolumn{6}{|l|}{ Model Summary for Transformed Response } \\
\hline$R-s q \quad R-s q(\operatorname{adj}) \quad R-s q(p$ & red) & & & & \\
\hline $\begin{array}{llll}0.524570 & 75.58 \% & 73.96 \% & 72\end{array}$ & $.07 \%$ & & & & \\
\hline
\end{tabular}

Figure 21: ANOVA results and P-values after removing additional insignificant factors, adjusted displacement.

All of the factors that remained in the model shown above in Figure 21 were significant with a $90 \%$ confidence level $(\alpha=0.1)$. The coefficient value calculated for the transformation was $\lambda=0.10$, which can be rounded to $\lambda=0$. Unusual observations that correspond to this model for adjusted displacement were as follows.

Table V: Notable outlier observations from adjusted displacement response

\begin{tabular}{|c|c|c|}
\hline Observation Number & Sample Name & Standardized Residual \\
\hline 90 & Control Tight High Nonlocking 1 & -4.56 \\
\hline 143 & PMMA Stripped Low Locking 5 & 3.14 \\
\hline 337 & CaP Stripped High Nonlocking 9 & -4.14 \\
\hline
\end{tabular}




\section{Load Strength}

Once again, a general linear model ANOVA was the best approach to accurately model the data for the Load Strength response. All factors and interactions through the $3^{\text {rd }}$ order were included.

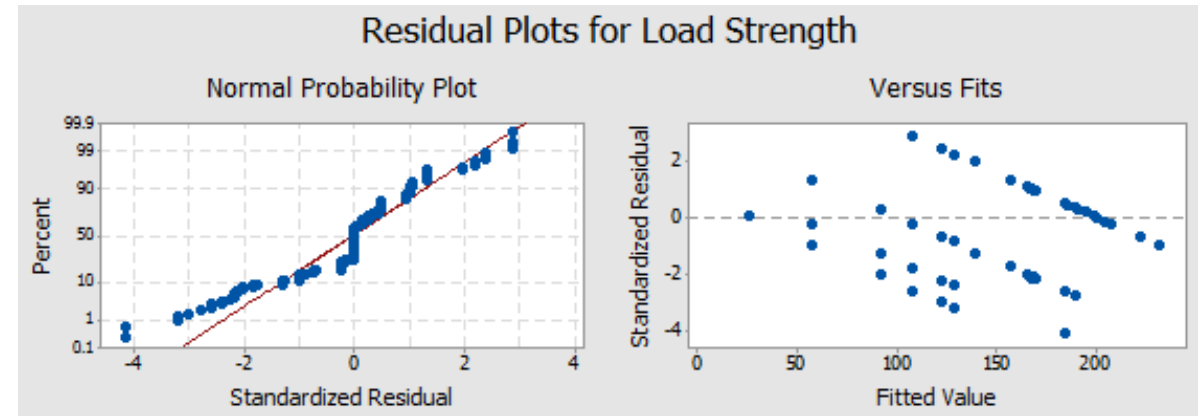

Figure 22: Probability plot and versus fit plot from general linear model ANOVA for Load Strength.

Minitab automatically removed the Cement*Hole size, Cement*Hole

Size*Density and Cement*Hole size*Screw Fixation interactions. The adjusted R-

squared value of this model was $73.00 \%$. However, the Versus Fits plot shown in Figure

22 above was relatively cone shaped so a transformation was implemented.

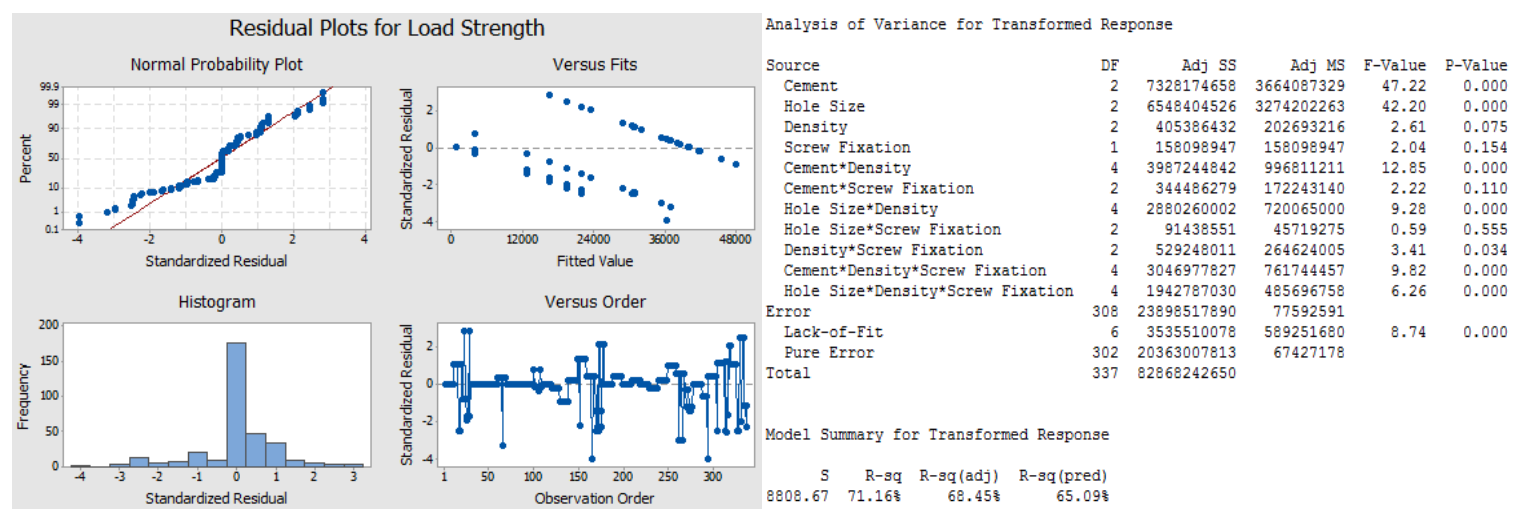


Figure 23: Residual plots for Load Strength response with a box-cox transformation (left) and resulting P-values (right), general linear model ANOVA.

The residuals didn't appear to be that much better after the transformation. The probability plot was still irregular and a cone shape was still portrayed in the versus fits plot. However, the transformation coefficient from the Box-Cox transformation was $\lambda=1.92$, which proves the transformation was indeed necessary for a more accurate model of the data. The adjusted R-squared value decreased to $68.45 \%$. Screw Fixation, Cement*Screw fixation and Hole Size*Screw Fixation factors all had a P-value over 0.180 so they can be removed from the model. Running the ANOVA again yielded:

\begin{tabular}{|c|c|c|c|c|c|}
\hline Source & $\mathrm{DF}$ & Adj Ss & Adj MS & F-Value & P-Value \\
\hline Cement & 2 & 14789918139 & 7394959069 & 93.78 & 0.000 \\
\hline Hole Size & 2 & 14317326215 & 7158663107 & 90.78 & 0.000 \\
\hline Density & 2 & 1270361769 & 635180884 & 8.05 & 0.000 \\
\hline Cement*Density & 4 & 5519052754 & 1379763188 & 17.50 & 0.000 \\
\hline Hole Size*Density & 4 & 4084061768 & 1021015442 & 12.95 & 0.000 \\
\hline Density*Screw Fixation & 2 & 455827117 & 227913559 & 2.89 & 0.057 \\
\hline Cement*Density*Screw Fixation & 4 & 3524891531 & 881222883 & 11.18 & 0.000 \\
\hline Hole Size*Density*Screw Fixation & 4 & 5250868124 & 1312717031 & 16.65 & 0.000 \\
\hline Error & 313 & 24681785883 & 78855546 & & \\
\hline Lack-of-Fit & 11 & 4318778070 & 392616188 & 5.82 & 0.000 \\
\hline Pure Error & 302 & 20363007813 & 67427178 & & \\
\hline Total & 337 & 82868242650 & & & \\
\hline \multicolumn{6}{|l|}{ Model Summary for Transformed Response } \\
\hline$S \quad R-s q \quad R-s q(\operatorname{adj}) \quad R-s q($ & & & & & \\
\hline $8880.06 \quad 70.22 \frac{8}{5} \quad 67.93 \frac{8}{5}$ & & & & & \\
\hline
\end{tabular}

Figure 24: Results of general linear model ANOVA, box-cox transformation for Load Strength response after removing all insignificant factors and factor interactions.

Remaining factors in the model shown in Figure 24 above were significant up to a $90 \%$ confidence level, $(\alpha=0.10)$. The adjusted R-squared value was $67.93 \%$ and the transformation coefficient was $\lambda=1.95$. Possible outliers within this model are listed in the following table. 
Table VI: Notable Outlier Observations from Load Strength Response

\begin{tabular}{|c|c|c|}
\hline Observation Number & Sample Name & Standardized Residual \\
\hline 65 & Control Stripped Medium Locking 6 & -3.20 \\
\hline 165 & PMMA Stripped Medium Locking 7 & -3.95 \\
\hline 294 & CaP Stripped Medium Locking 1 & -3.95 \\
\hline
\end{tabular}

\section{Stiffness}

The fourth and final response was Stiffness $(\mathrm{N} / \mathrm{mm})$. A general linear model ANOVA with all two-way and three-way interactions was again used for the Stiffness response.
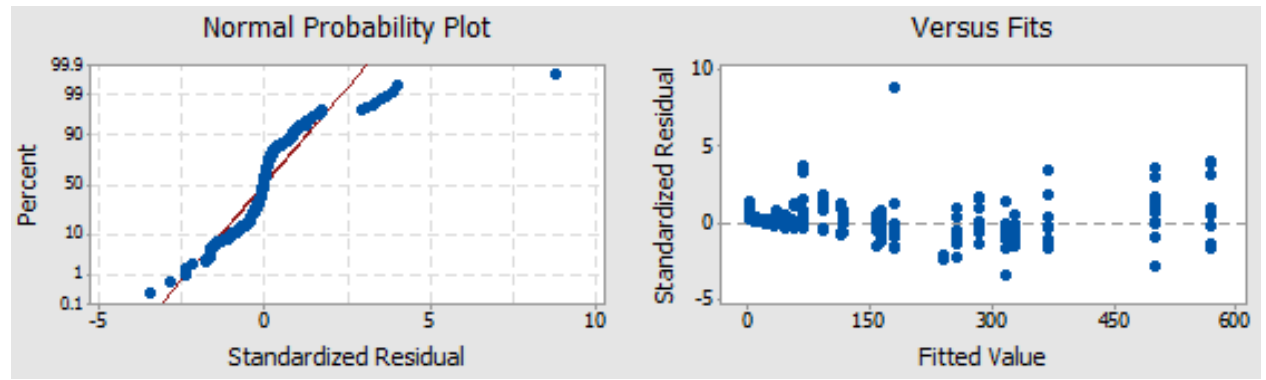

Figure 25: Residual plots of the Stiffness response from a general linear model ANOVA.

With an adjusted R-squared value of $72.38 \%$, this model, shown above in Figure 25 appeared to be in need of a transformation, as was the trend with this data. The Probability plot was hardly linear and the Versus Fits plot was cone shaped. Also worth noting is that Minitab once again automatically removed Cement*Hole size, Cement*Hole Size*Density and Cement*Hole size*Screw Fixation interactions. These terms could not be estimated and were removed from all four general linear models. 


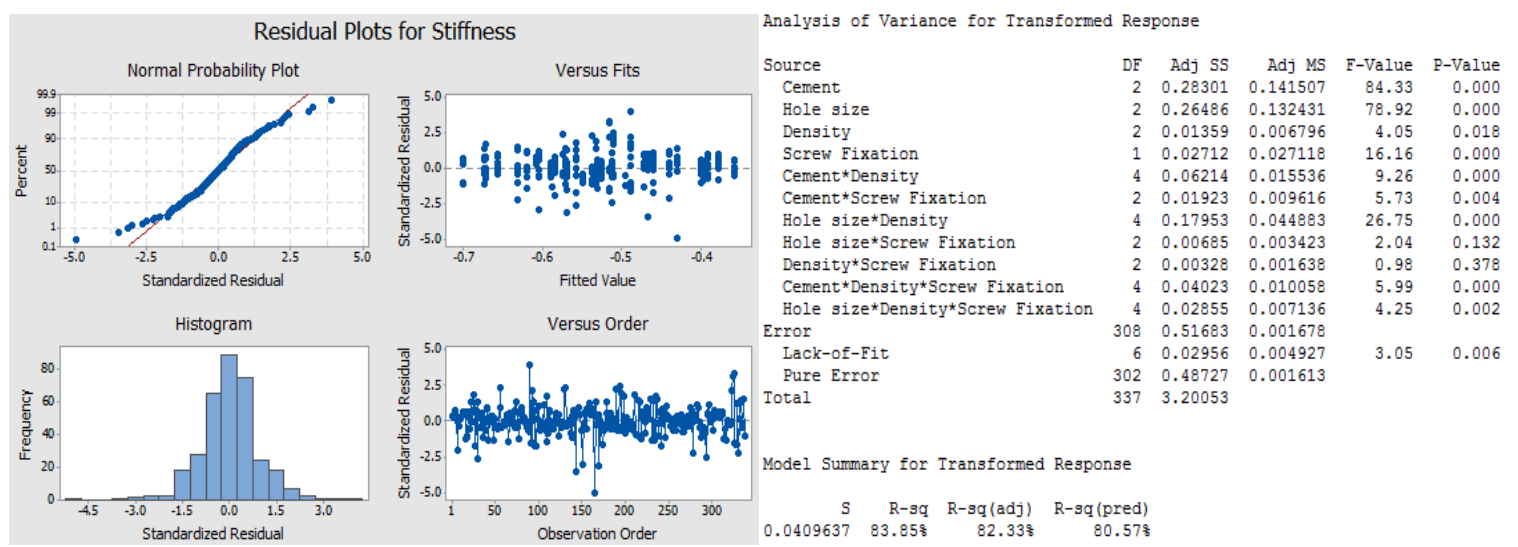

Figure 26: Residual plots (left) and P-values (right) for general linear model ANOVA with boxcox transformation for Stiffness response.

A Box-Cox transformation once again helped shape the data into an accurate

looking linear model, as shown above in Figure 26. The adjusted R-squared value increased from $72.38 \%$ to $82.33 \%$ and the residual plots (above, left) were much more linear. Density*Screw Fixation and Hole Size*Screw Fixation were not significant at $\alpha=0.10$, so they were removed from the model.

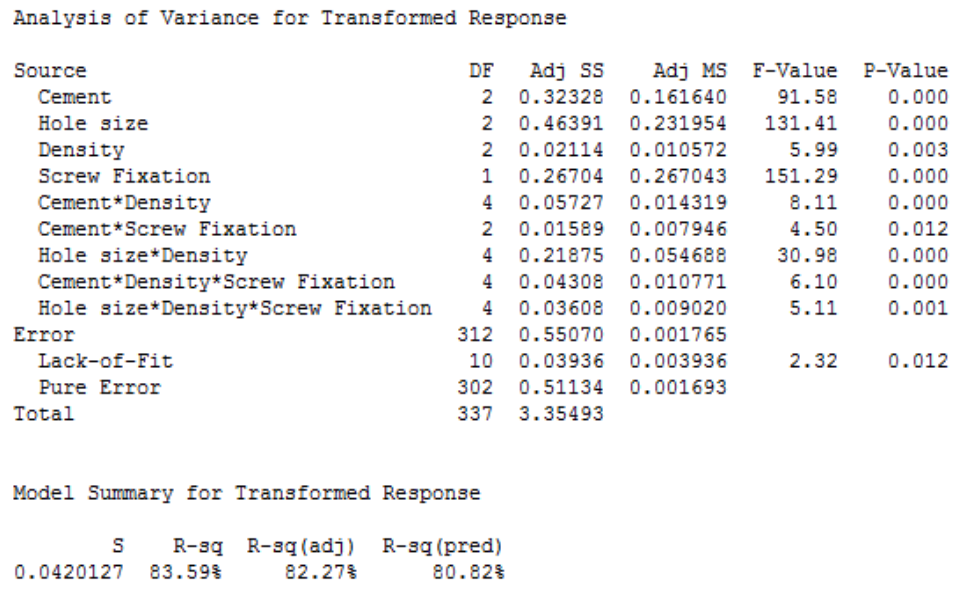

Figure 27: Results of general linear model ANOVA, box-cox transformation for Stiffness response after removing all insignificant factors and factor interactions. 
All terms in the model shown above in Figure 27 were now significant $(\alpha=0.05)$.

The box-cox transformation coefficient was $\lambda=-0.17$. Possible outliers can now be noted from this optimal general linear model.

Table VII: Notable Outlier Observations from Stiffness Response

\begin{tabular}{|c|c|c|}
\hline Observation Number & Sample Name & Standardized Residual \\
\hline 90 & Control Tight High Nonlocking 1 & 3.55 \\
\hline 143 & PMMA Stripped Low Locking 5 & -3.54 \\
\hline 165 & PMMA Stripped Medium Locking 7 & -4.93 \\
\hline 170 & PMMA Stripped Medium Nonlocking 2 & -3.22 \\
\hline 325 & CaP Stripped High Locking 6 & 3.05 \\
\hline 326 & CaP Stripped High Locking 7 & 3.19 \\
\hline
\end{tabular}

Combining the unusual residuals from the four models calculated above resulted in a full list of possible outliers for this study.

Table VIII: Notable Outlier Observations Across all Models, First Round

\section{Cycles Survived}

\begin{tabular}{|c|c|c|}
\hline Observation number & Sample Name & Standardized Residual \\
\hline 26 & Control Stripped Low Locking 6 & -6.57 \\
\hline 106 & Control Stripped High Locking 7 & -3.12 \\
\hline 165 & PMMA Stripped Medium Locking 7 & -3.70 \\
\hline 170 & PMMA Stripped Medium Nonlocking 2 & -5.34 \\
\hline 173 & PMMA Stripped Medium Nonlocking 5 & -4.96 \\
\hline 274 & CaP Stripped Low Nonlocking 7 & -3.09 \\
\hline 294 & CaP Stripped Medium Locking 1 & -3.68 \\
\hline 337 & CaP Stripped High Nonlocking 9 & -5.04 \\
\hline
\end{tabular}

\section{Adjusted Displacement}

\begin{tabular}{|c|c|c|}
\hline Observation Number & Sample Name & Standardized Residual \\
\hline 90 & Control Tight High Nonlocking 1 & -4.56 \\
\hline 143 & PMMA Stripped Low Locking 5 & 3.14 \\
\hline 337 & CaP Stripped High Nonlocking 9 & -4.14 \\
\hline
\end{tabular}




\section{Load Strength}

\begin{tabular}{|c|c|c|}
\hline Observation Number & Sample Name & Standardized Residual \\
\hline 65 & Control Stripped Medium Locking 6 & -3.20 \\
\hline 165 & PMMA Stripped Medium Locking 7 & -3.95 \\
\hline 294 & CaP Stripped Medium Locking 1 & -3.95 \\
\hline
\end{tabular}

\section{Stiffness}

\begin{tabular}{|c|c|c|}
\hline Observation Number & Sample Name & Standardized Residual \\
\hline 90 & Control Tight High Nonlocking 1 & -3.55 \\
\hline 143 & PMMA Stripped Low Locking 5 & 3.54 \\
\hline 165 & PMMA Stripped Medium Locking 7 & 4.93 \\
\hline 170 & PMMA Stripped Medium Nonlocking 2 & 3.22 \\
\hline 325 & CaP Stripped High Locking 6 & -3.05 \\
\hline 326 & CaP Stripped High Locking 7 & -3.19 \\
\hline
\end{tabular}

Considering the tables shown above in Table VII, not all listed samples will be omitted from the data set. Removing too much data can negatively affect the entire data set, and this author was hesitant to omit a data point that was observed as showing adequate testing behavior. After careful consideration, the following samples were selected as outliers:

- Control Stripped Low Locking 6

- Control Tight High Nonlocking 1

- $\quad$ PMMA Stripped Low Locking 5

- $\quad$ PMMA Stripped Medium Locking 7

- $\quad$ PMMA Stripped Medium Nonlocking 2

- $\quad$ PMMA Stripped Medium Nonlocking 5

- CaP Stripped Medium Locking 1

- $\quad$ CaP Stripped High Nonlocking 9 
Since the 8 samples listed above had been deemed statistically outlying from the rest of the data, they were removed from the model. The linear ANOVA was calculated again using the same optimal models as shown above and outliers from the resulting model were noted and the process was repeated below.

\section{E. Cycles Survived Round Two}

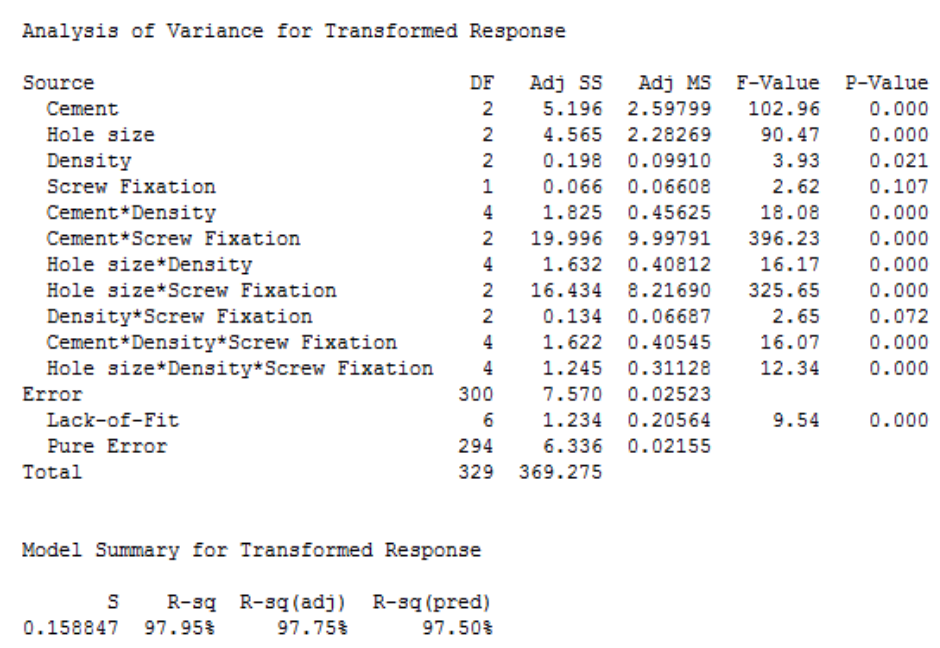

Figure 28: General linear model ANOVA for the Cycles Survived response after removing 8 statistically outlying samples.

The box-cox transformation coefficient was now $\lambda=0.19$, it was $\lambda=0.5$ before

omitting the first round of outliers. The adjusted R-squared percentage jumped up to $97.75 \%$. All but one of the factors were still significant with $90 \%$ confidence, $\alpha=0.10$. No additional changes were needed. A standard residual of \pm 3.5 will be used as the threshold for a notable outlier moving forward. 
Table IX: Notable outliers for Cycles Survived response, round two

\begin{tabular}{|c|c|c|}
\hline Observation Number & Sample Name & Standardized Residual \\
\hline 104 & Control Stripped High Locking 7 & -5.80 \\
\hline 166 & PMMA Stripped Medium Nonlocking 3 & -3.80 \\
\hline 170 & PMMA Stripped Medium Nonlocking 8 & -4.19 \\
\hline 268 & CaP Stripped Low Nonlocking 7 & -5.02 \\
\hline 269 & CaP Stripped Low Nonlocking 8 & -3.88 \\
\hline 325 & CaP Stripped High Nonlocking 3 & -3.63 \\
\hline
\end{tabular}

\section{F. Adjusted Displacement Round Two}

$\begin{array}{lrrrrr}\text { Analysis of Variance for Transformed Response } & & \\ & \text { DF } & \text { Adj SS } & \text { Adj MS } & \text { F-Value } & \text { P-Value } \\ \text { Source } & 2 & 37.621 & 18.8106 & 85.02 & 0.000 \\ \quad \text { Cement } & 2 & 17.283 & 8.6417 & 39.06 & 0.000 \\ \text { Hole size } & 1 & 26.347 & 26.3472 & 119.09 & 0.000 \\ \text { Screw Fixation } & 4 & 15.643 & 3.9107 & 17.68 & 0.000 \\ \text { Cement*Density } & 2 & 38.755 & 19.3775 & 87.59 & 0.000 \\ \text { Cement*Screw Fixation } & 4 & 25.409 & 6.3523 & 28.71 & 0.000 \\ \text { Hole size*Density } & 2 & 11.784 & 5.8918 & 26.63 & 0.000 \\ \text { Hole size*Screw Fixation } & 4 & 3.682 & 0.9206 & 4.16 & 0.003 \\ \text { Hole size*Density*Screw Fixation } & 308 & 68.141 & 0.2212 & & \\ \text { Error } & 14 & 12.057 & 0.8612 & 4.51 & 0.000 \\ \quad \text { Lack-of-Fit } & 294 & 56.084 & 0.1908 & & \\ \quad \text { Pure Error } & 329 & 344.116 & & & \\ \text { Total } & & & & & \\ & & & & & \end{array}$

Model Summary for Transformed Response

$\begin{array}{rrrr}\text { S } & \text { R-sq } & \text { R-sq(adj) } & \text { R-sq (pred) } \\ 0.470359 & 80.20 \% & 78.85 \% & 77.398\end{array}$

Figure 29: General linear model ANOVA for the Adjusted Displacement response after removing 8 statistically outlying samples.

The box-cox transformation coefficient was $\lambda=0.04$, compared to $\lambda=0.10$ before the first round of outliers was removed and the R-squared value increased from $73.96 \%$ to $78.85 \%$. All of the factors this time around were significant up to $99 \%$ confidence, $\alpha=0.01$. No samples had a standardized residual of \pm 3.5 for this response. 


\section{G. Load Strength Round Two}

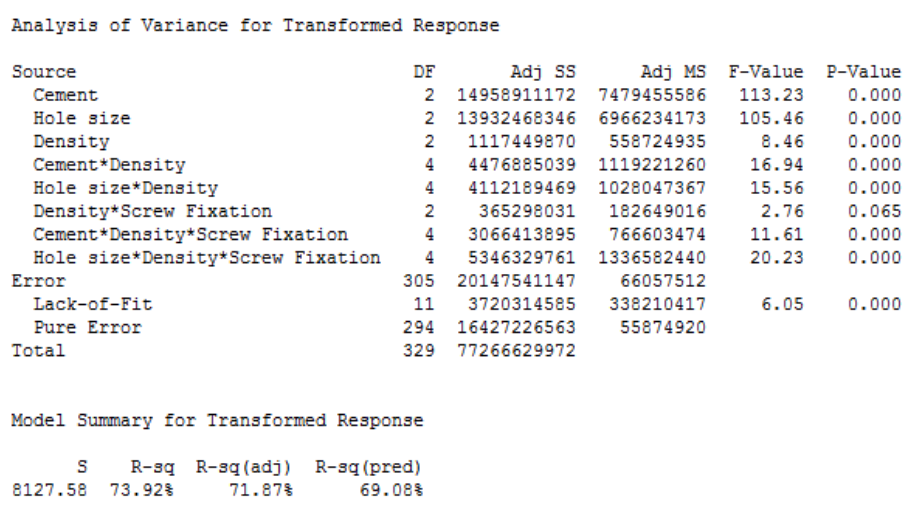

Figure 30: General linear model ANOVA for Load Strength response after removing 8 statistically outlying samples.

The adjusted R-squared value increased from $67.93 \%$ to $71.87 \%$ and the transformation coefficient slightly decreased from $\lambda=1.95$ to $\lambda=1.94$.

Table X: Notable outliers for Load Strength response, round two

\begin{tabular}{|c|c|c|}
\hline Observation Number & Sample Name & Standardized Residual \\
\hline 64 & Control Stripped Medium Locking 6 & -3.50 \\
\hline
\end{tabular}




\section{H. Stiffness Round Two}

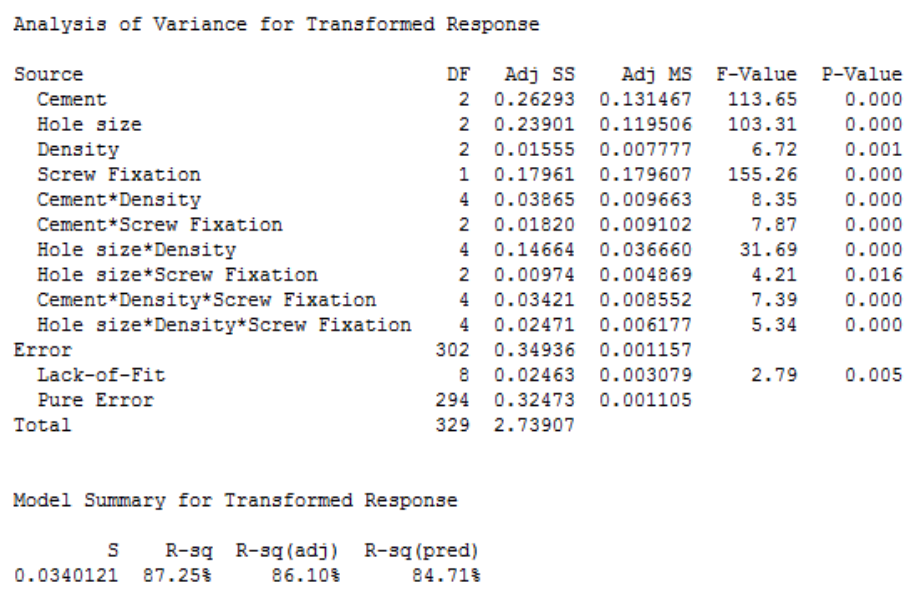

Figure 31: General linear model ANOVA for the Stiffness response after removing 8 statistically outlying samples.

The new box-cox transformation coefficient was $\lambda=-0.14$, which is not much different than $\lambda=-0.17$ from the first round of modeling. R-squared increased from $82.27 \%$ to $86.10 \%$.

Table XI: Notable outliers for Stiffness, round two

\begin{tabular}{|c|c|c|}
\hline Observation Number & Sample Name & Standardized Residual \\
\hline 318 & CaP Stripped High Locking 6 & 3.51 \\
\hline 319 & CaP Stripped High Locking 7 & 3.67 \\
\hline
\end{tabular}

Only two samples had a noteworthy residual this time around, and they were samples that showed up on the list from the first round of modeling that didn't make the cut. Of the 9 samples listed above in tables IX, $\mathrm{X}$ and XI, none had adequate residuals to merit an omission. The results in figures $28,29,30$, and 31 correspond to the final models for responses Cycles Survived, Adjusted Displacement, Load Strength and Stiffness 
respectively. They are also shown side by side below. The final dataset has a total of 330

\section{samples.}

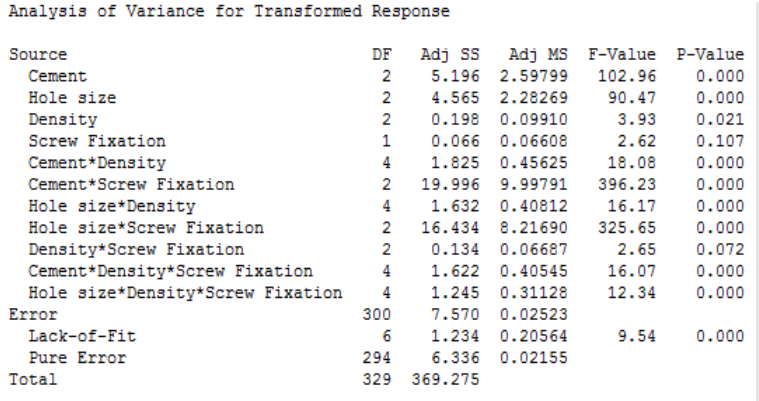

Model Summary for Transformed Response

$\begin{array}{rrrr}\text { S } & \text { R-sq } & \text { R-sq (adj) } & \text { R-sq (pred) } \\ 0.158847 & 97.958 & 97.75 \% & 97.508\end{array}$

Analysis of Variance for Transformed Response

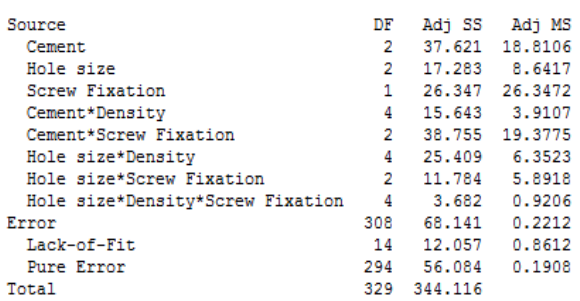

Model Surmary for Transformed Response

$\begin{array}{rrrr}\text { S } & \text { R-sq } & \text { R-sq (adj) } & \text { R-sq (pred) } \\ 0.470359 & 80.208 & 78.85 \% & 77.39 \%\end{array}$

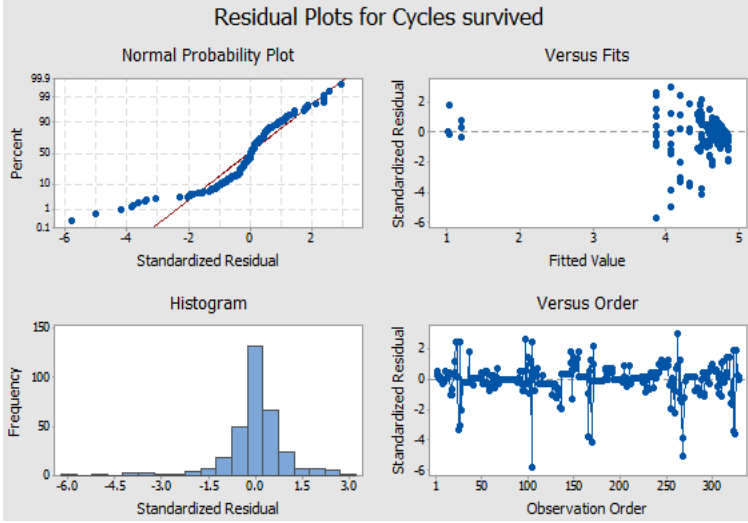

Residual Plots for Adjusted Displacement

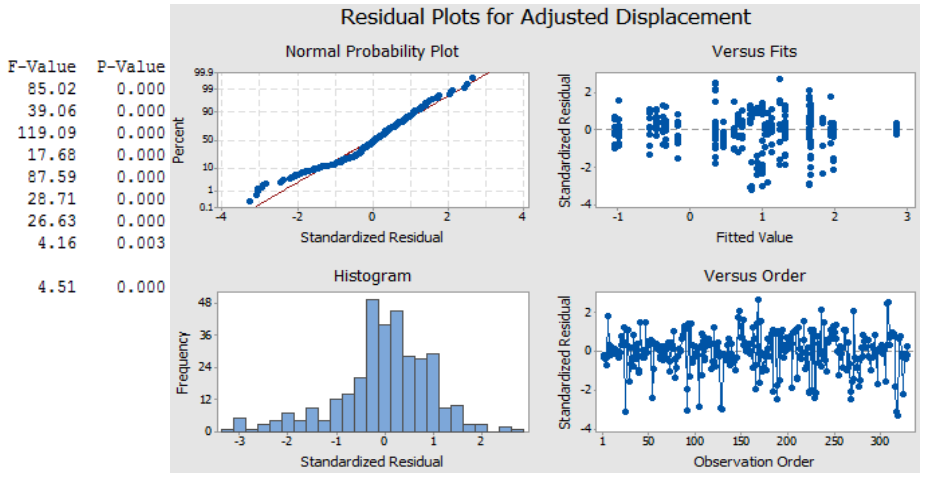

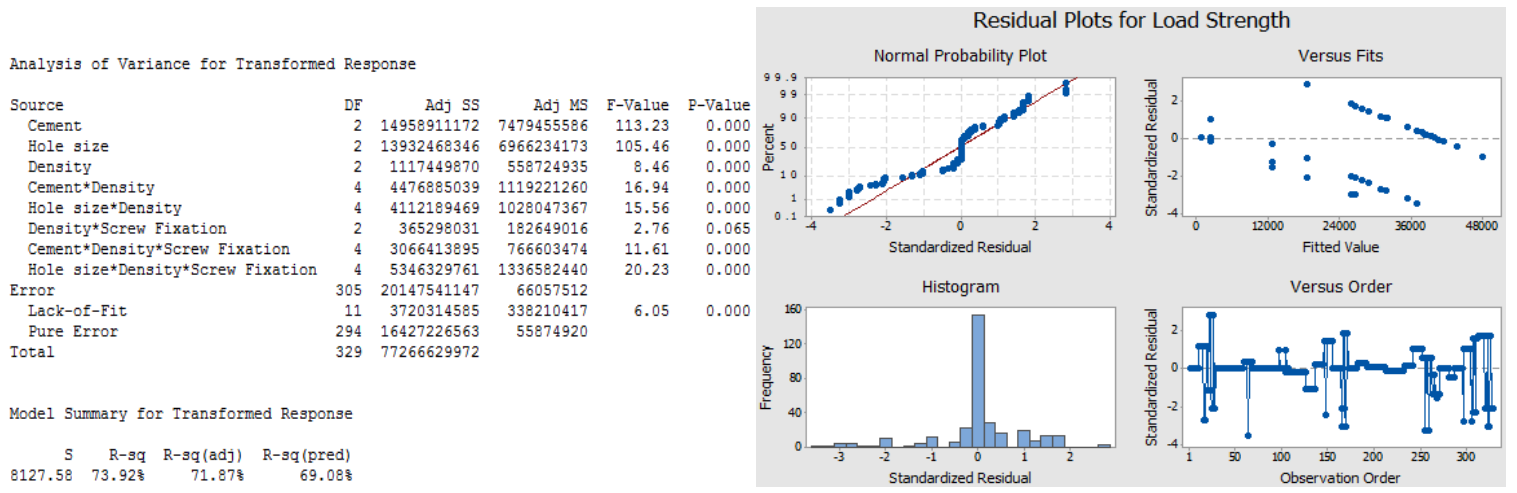




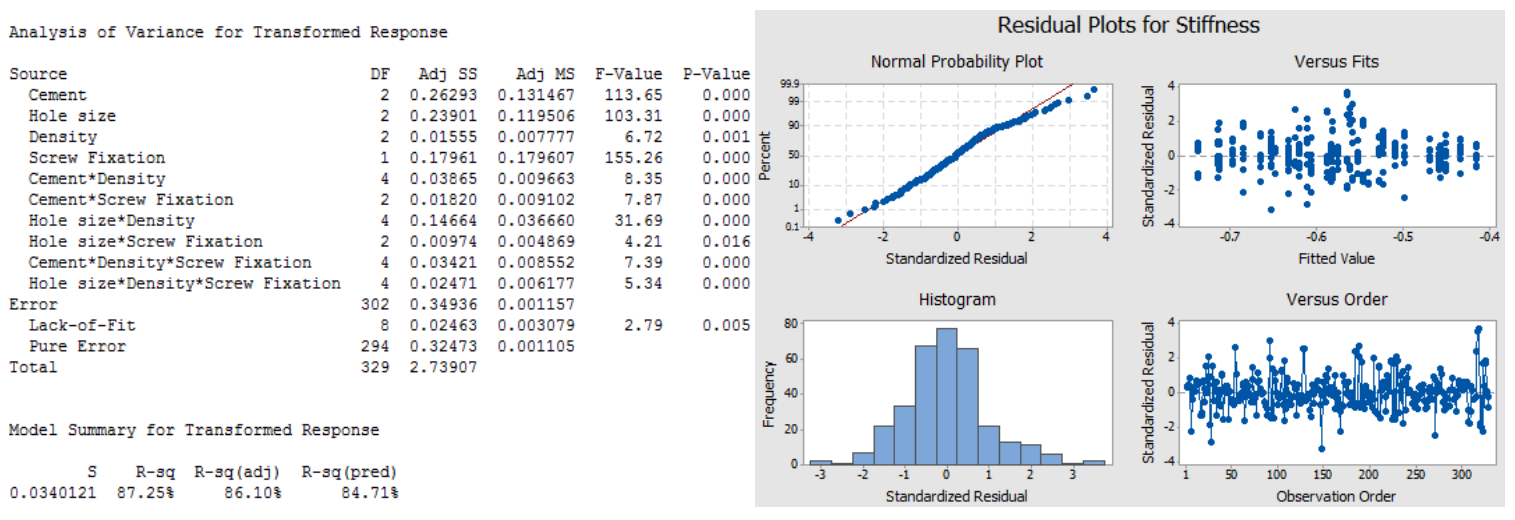

Figure 32: Final general linear model ANOVA results (left column) and resulting standardized residuals (right column) for Cycles Survived (top) Adjusted Displacement (top middle) Load Strength (bottom middle) and Stiffness (bottom) responses with all outliers removed. A Box-cox transformation was used for all models shown above. 


\section{RESULTS}

As stated above in the Statistical Analysis, the four responses analyzed for this study were cycles survived, adjusted displacement, displacement over load, and stiffness. The Cycles Survived response is self-explanatory; the number of cycles the screw remained intact inside the foam block on a scale of 1-4000. Adjusted displacement is the measured vertical displacement of the last 10 cycles of the test before failure (or cycles $3990-4000$ if the screw survived) minus the predetermined "slop" displacement for that load level, as described above. The Load Strength response is simply the load level recorded when the sample screw failed, which can be one of the four load levels shown above. Lastly, stiffness $(\mathrm{N} / \mathrm{mm})$ is the load level at time of failure divided by the adjusted displacement. This response was an attempt at normalizing all the displacement data across multiple different factors in the study. Stiffness values range between $0-1000$.

\section{A. Control Group No Cement}

Listed below are all averaged responses for every sample in this study, sorted first by cement type and then by density. The Cycles Completed, Stiffness and Load Strength responses were straight forward with averages and standard deviations shown below, along with the percentage of samples that failed (out of 10 or less) for each factor 
combination under the Cycles Completed table. However, adjusted displacement was more complicated since it didn't take into account the load level at failure. This response was simply the average of the differences of the last 10 cycles survived for each sample, regardless of load level or cycles completed up to that point. So if a screw pulled out almost immediately it will inherently have very little displacement, compared to a screw that hung on for $3000+$ cycles into the $200 \mathrm{~N}$ load level that had a displacement in the normal range. The first screw, when comparing only adjusted displacement, will have had less motion and therefore be deemed intuitively "better" than the second screw in this example, when in reality the second screw survived much longer than the first. To counter this, the adjusted displacement response averages in the following figures were normalized to a single load level $(100 \mathrm{~N})$ for each factor.

\begin{tabular}{|c|c|c|c|c|c|c|c|c|c|}
\hline \multicolumn{5}{|c|}{ Adj Displacement (mm) 100N Low Density Control } & \multicolumn{5}{|c|}{ Load Strength (N) Low Density Control } \\
\hline & \multicolumn{2}{|c|}{ Locking } & \multicolumn{2}{|c|}{ Nonlocking } & & \multicolumn{2}{|c|}{ Locking } & \multicolumn{2}{|c|}{ Nonlocking } \\
\hline & Tight & Stripped* & Tight & Stripped & & Tight & Stripped* & Tight & Stripped \\
\hline mean & 1.11 & 3.10 & 7.01 & - & mean & 200 & 118.75 & 170 & 25 \\
\hline \multirow[t]{2}{*}{ stdev } & 0.57 & 0.17 & 6.43 & - & stdev & 0 & 70.39 & 48.30 & 0 \\
\hline & \multicolumn{2}{|c|}{ *excludes outliers $(6,10)$} & & & & \multicolumn{2}{|c|}{ * excludes outlier $(6,10)$} & & \\
\hline
\end{tabular}

\begin{tabular}{|l|c|c|c|c|c|c|c|c|}
\hline \multicolumn{9}{|c|}{ Cycles completed Low Density Control } \\
\hline
\end{tabular}

Figure 33: Adjusted Displacement at 100N load level (top left) Load Strength (top right) Cycles Completed (bottom left) and Stiffness (bottom right) for control group, low density. The Displacement averages (top left) were normalized to a single load level of $100 \mathrm{~N}$ rather than only showing the displacement at failure across the board. Different factor combinations (tight locking vs stripped nonlocking) often resulted in screw samples failing in different load levels, which would give skewed results if portrayed all in the same table. 

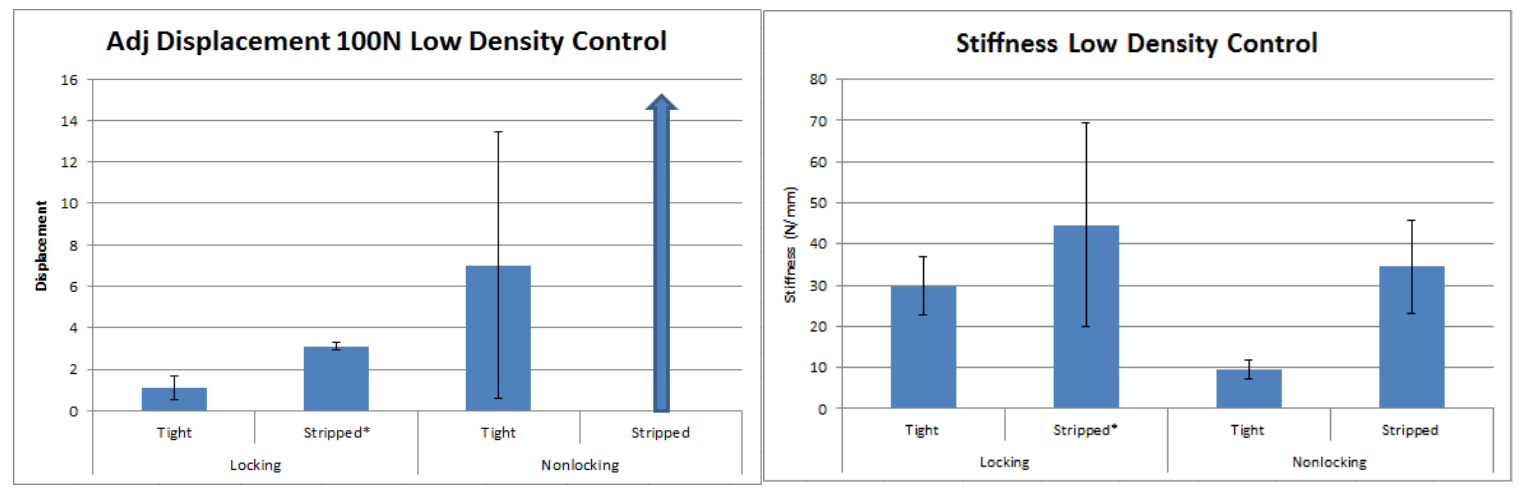

Figure 34: Adjusted displacement averages at 100N load level (left) and Stiffness (right) low density control. All control samples in the low density failed at some point. The arrow denotes all screws failed before $100 \mathrm{~N}$ load level.
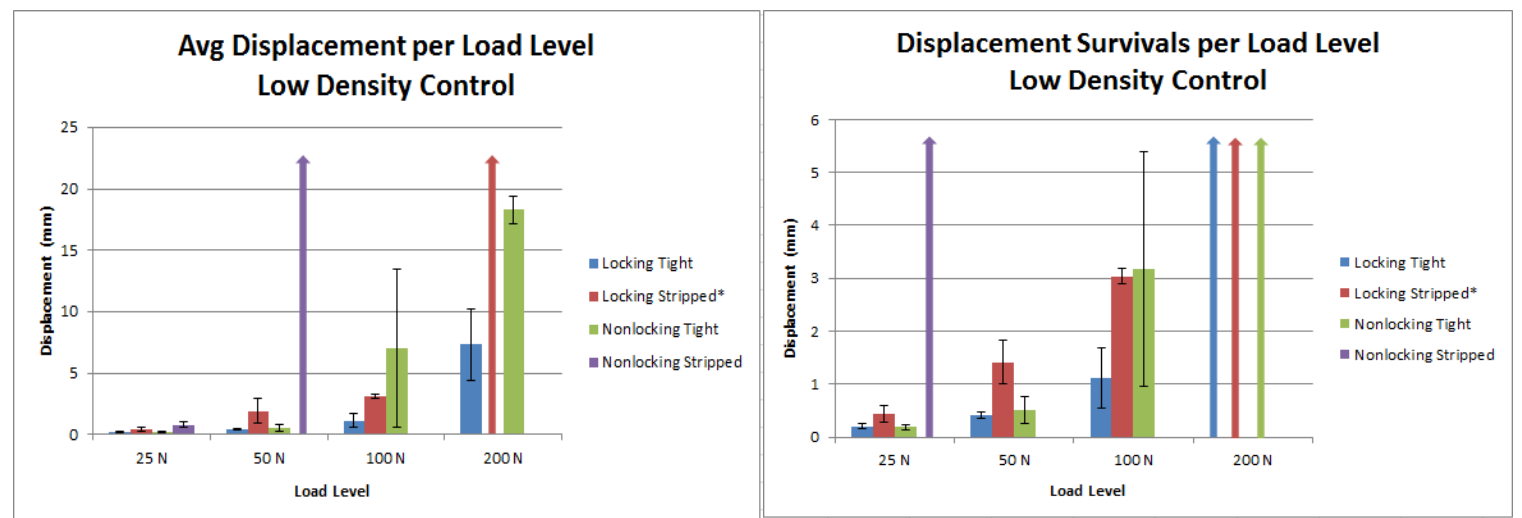

Figure 35: Adjusted displacement per load level, low density control. The right side graph shows displacements only for screws that survived the full 1000 cycles at the respective load level. The left side graph shows averaged displacements at each load level regardless of whether the sample failed at that load level or not. Arrows on either side denote all screws failed before current load level, or before 1000 cycles were completed. 


\begin{tabular}{|c|c|c|c|c|c|c|c|c|c|}
\hline \multicolumn{5}{|c|}{ Adj Displacement (mm) 100N Medium Density Control } & \multicolumn{5}{|c|}{ Load Strength (N) Medium Density Control } \\
\hline & \multicolumn{2}{|c|}{ Locking } & \multicolumn{2}{|c|}{ Nonlocking } & & \multicolumn{2}{|c|}{ Locking } & \multicolumn{2}{|c|}{ Nonlocking } \\
\hline & Tight & Stripped & Tight & Stripped & & Tight & Stripped & Tight & Stripped \\
\hline mean & 0.74 & 1.92 & 1.00 & - & Mean & 200.0 & 190.0 & 200.0 & 25.0 \\
\hline stdev & 0.45 & 0.75 & 0.66 & - & Stdev & 0.0 & 31.62 & 0.0 & 0.0 \\
\hline
\end{tabular}

\begin{tabular}{|c|c|c|c|c|c|c|c|c|c|}
\hline \multicolumn{5}{|c|}{ Cycles completed Medium Density Control } & & & & & \\
\hline & \multicolumn{2}{|c|}{ Locking } & \multicolumn{2}{|c|}{ Nonlocking } & \multicolumn{5}{|c|}{ Stiffness (N/mm) Medium Density Control } \\
\hline & Tight & Stripped & Tight & Stripped & & \multicolumn{2}{|c|}{ Locking } & \multicolumn{2}{|c|}{ Nonlocking } \\
\hline Mean & 3968.7 & 3282.3 & 3698.5 & 1.0 & & Tight & Stripped & Tight & Stripped \\
\hline Stdev & 93.8 & 216.8 & 281.9 & 0.0 & Mean & 115.84 & 53.53 & 35.18 & 12.07 \\
\hline$\%$ Fail & 20 & 100 & 80 & 100 & Stdev & 39.56 & 11.33 & 21.07 & 3.33 \\
\hline
\end{tabular}

Figure 36: Adjusted Displacement at 100N load level (top left) Load Strength (top right) Cycles Survived (bottom left) and Stiffness (bottom right) for control group, medium density. The Displacement averages (top left) were normalized to a single load level of $100 \mathrm{~N}$ rather than only showing the displacement at failure across the board. Different factor combinations (tight locking vs stripped nonlocking) often resulted in screw samples failing in different load levels, which would give skewed results if portrayed all in the same table.
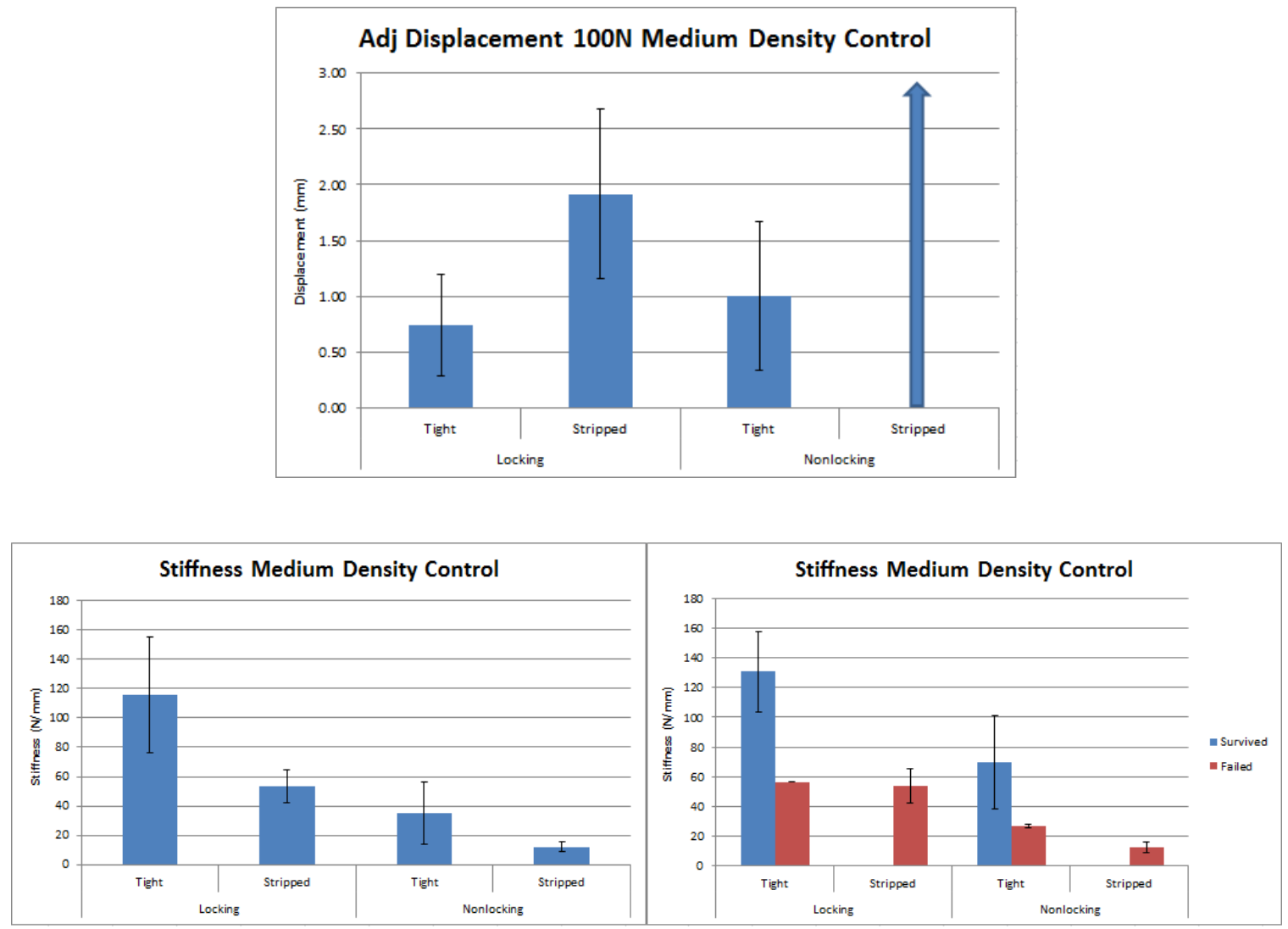

Figure 37: Adjusted displacement averages at 100N load level (top) and Stiffness (bottom row) control group, medium density. The bottom right graph shows the same Stiffness (bottom left) values but with sample survivals and failures separated for better visualization. The arrow in the top graph denotes all screws failed before the $100 \mathrm{~N}$ load level. 


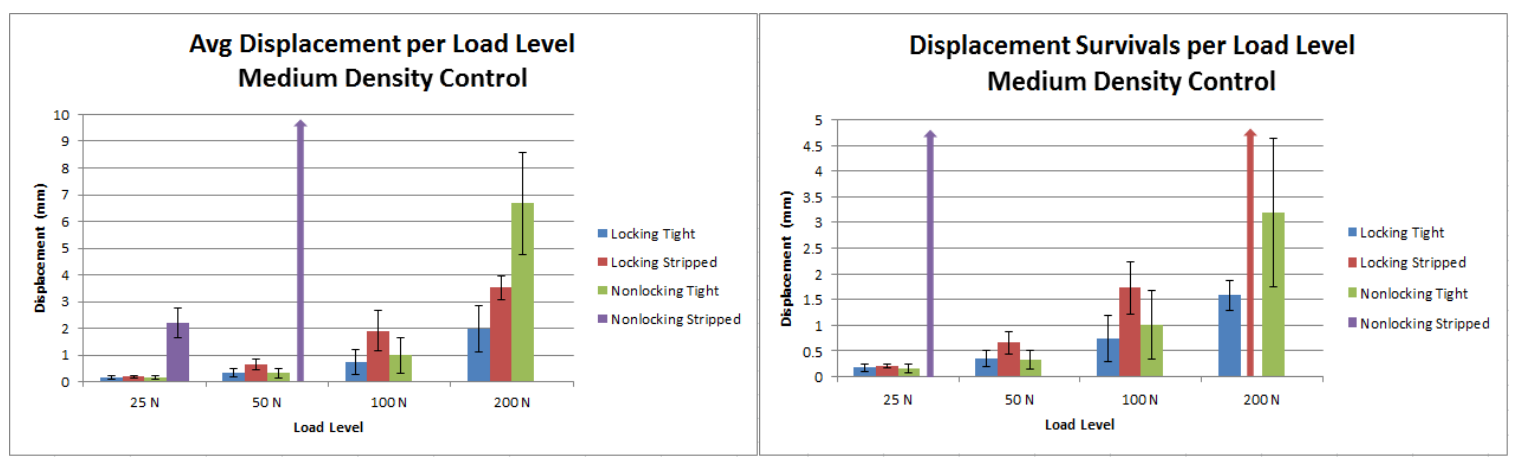

Figure 38: Adjusted displacement per load level for all samples (left) and survivals only (right) control group, medium density. The right side graph shows displacements only for screws that survived the full 1000 cycles at the respective load level. The left side graph shows averaged displacements at each load level regardless of whether the sample failed at that load level or not. Arrows denote all screw samples failed before or during load level.

\begin{tabular}{|c|c|c|c|c|c|c|c|c|c|}
\hline \multicolumn{5}{|c|}{ Adj Displacement (mm) 100N High Density Control } & \multicolumn{5}{|c|}{ Load Strength (N) High Density Control } \\
\hline & \multicolumn{2}{|c|}{ Locking } & \multicolumn{2}{|c|}{ Nonlocking } & & \multicolumn{2}{|c|}{ Locking } & \multicolumn{2}{|c|}{ Nonlocking } \\
\hline & Tight & Stripped & Tight* & Stripped & & Tight & Stripped & Tight* & Stripped \\
\hline mean & 0.38 & 3.37 & 0.71 & - & Mean & 200.00 & 57.50 & 200.00 & 25.00 \\
\hline \multirow[t]{2}{*}{ stdev } & 0.15 & - & 0.26 & - & Stdev & 0.00 & 23.72 & 0.00 & 0.00 \\
\hline & \multicolumn{2}{|c|}{ *excludes outlier (1) } & & & & \multicolumn{2}{|c|}{ *excludes outlier (1) } & & \\
\hline
\end{tabular}

\begin{tabular}{|c|c|c|c|c|c|c|c|c|c|}
\hline \multicolumn{5}{|c|}{ Cycles completed High Density Control } & & & & & \\
\hline & \multicolumn{2}{|c|}{ Locking } & \multicolumn{2}{|c|}{ Nonlocking } & \multicolumn{5}{|c|}{ Stiffness (N/mm) High Density Control } \\
\hline & Tight & Stripped & Tight* & Stripped & & \multicolumn{2}{|c|}{ Locking } & \multicolumn{2}{|c|}{ Nonlocking } \\
\hline Mean & 4000 & 1342.7 & 3703.2 & 2.6 & & Tight & Stripped & Tight* & Stripped \\
\hline Stdev & 0 & 514.5 & 317.7 & 0.7 & Mean & 368.61 & 20.84 & 98.50 & 12.43 \\
\hline \multirow[t]{2}{*}{$\%$ Fail } & 0 & 100 & 50 & 100 & Stdev & 130.07 & 7.16 & 85.35 & 4.15 \\
\hline & \multicolumn{2}{|c|}{ *excludes outlier (1) } & & & & \multicolumn{2}{|c|}{ *excludes outlier (1) } & & \\
\hline
\end{tabular}

Figure 39: Adjusted Displacement at $100 \mathrm{~N}$ load level (top left) Load Strength (top right) Cycles Survived (bottom left) and Stiffness (bottom right) for control group, high density. The Displacement averages (top left) were normalized to a single load level of $100 \mathrm{~N}$ rather than only showing the displacement at failure across the board. Different factor combinations (tight locking vs stripped nonlocking) often resulted in screw samples failing in different load levels, which would give skewed results if portrayed all in the same table. 

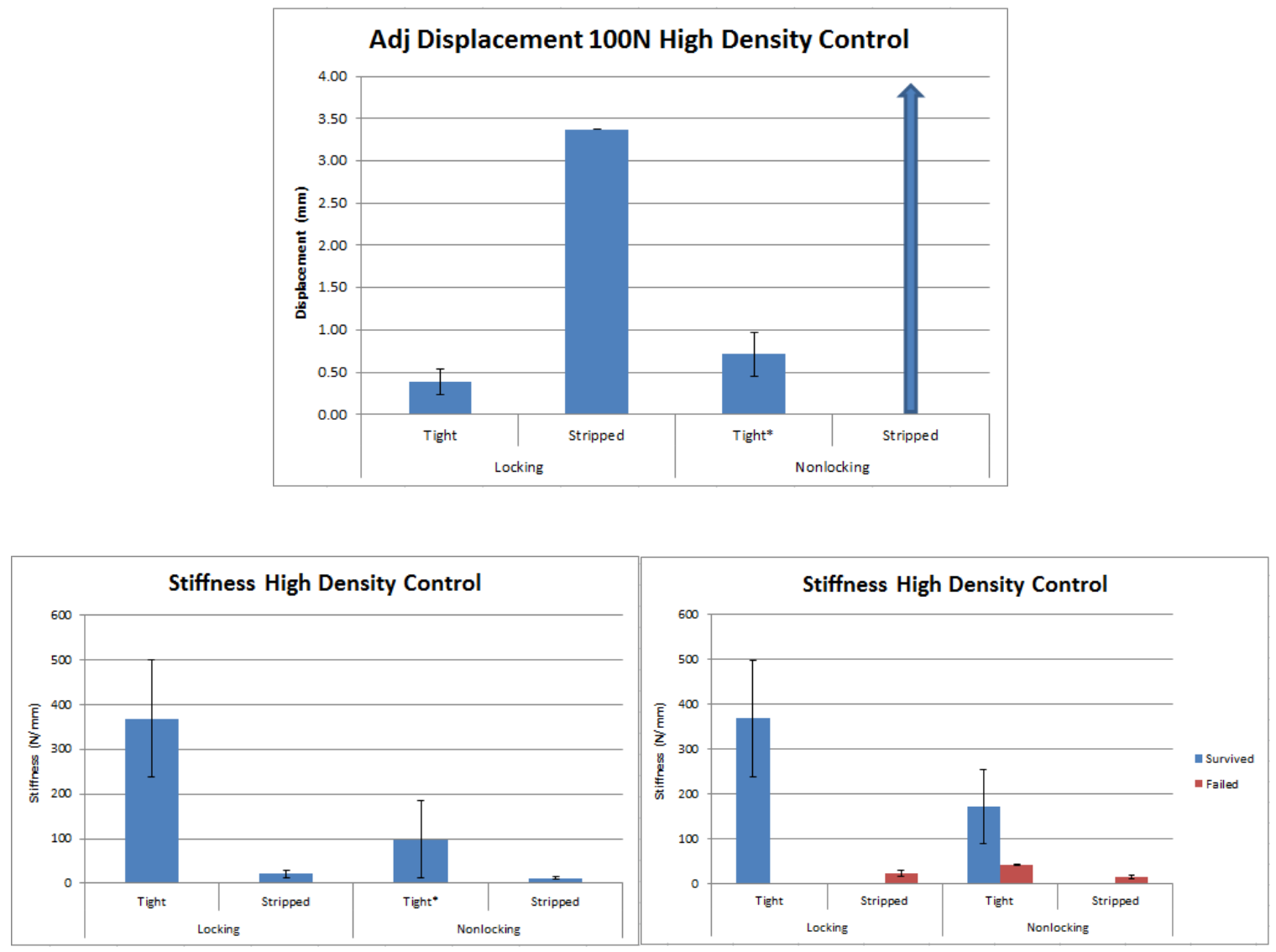

Figure 40: Adjusted Displacement averages at the 100N load level (top) and Stiffness (bottom row) control group, high density. The bottom right graph shows the same Stiffness (bottom left) values but with sample survivals and failures separated for better visualization. The arrow in the top graph denotes all screws failed before the $100 \mathrm{~N}$ load level.
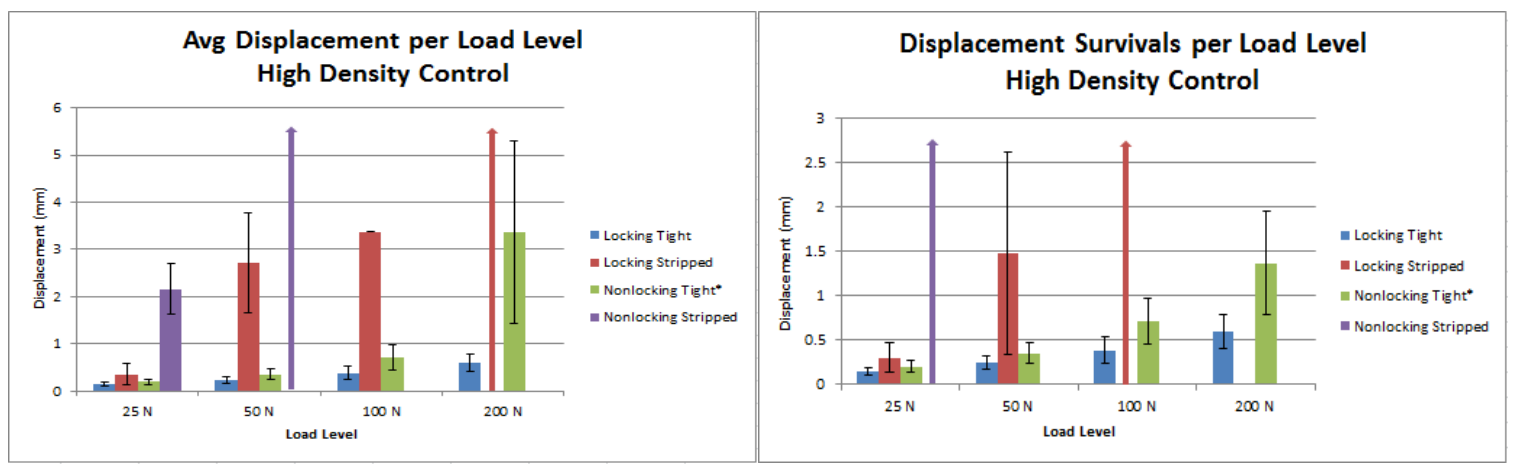

Figure 41: Adjusted Displacement per load level, all samples (left) and screw survivals only (right) control group high density. The right side graph shows displacements only for screws that survived the full 1000 cycles at the respective load level. The left side graph shows averaged displacements at each load level regardless of whether the sample failed at that load level or not. Arrows denote all screw samples failed before or during load level. 


\section{B. PMMA Cement Group}

\begin{tabular}{|c|c|c|c|c|c|c|c|c|c|}
\hline \multicolumn{5}{|c|}{ Adj Displacement (mm) 100N Low Density PMMA } & \multicolumn{5}{|c|}{ Load Strength (N) Low Density PMMA } \\
\hline & \multicolumn{2}{|c|}{ Locking } & \multicolumn{2}{|c|}{ Nonlocking } & & \multicolumn{2}{|c|}{ Locking } & \multicolumn{2}{|c|}{ Nonlocking } \\
\hline & Large* & Stripped** & Large & Stripped & & Large* & Stripped** & Large & Stripped \\
\hline mean & 0.42 & 0.64 & 0.95 & 2.18 & Mean & 200.0 & 200.0 & 200.0 & 190.0 \\
\hline \multirow[t]{3}{*}{ stdev } & 0.15 & 0.16 & 0.42 & 0.84 & Stdev & 0.0 & 0.0 & 0.0 & 31.62 \\
\hline & \multicolumn{2}{|c|}{ *excludes outlier (4) } & & & & \multicolumn{2}{|c|}{ *excludes outlier (4) } & & \\
\hline & \multicolumn{2}{|c|}{ **excludes outlier (5) } & & & & \multicolumn{2}{|c|}{ **excluding outlier (5) } & & \\
\hline
\end{tabular}

\begin{tabular}{|c|c|c|c|c|c|c|c|c|c|}
\hline \multicolumn{5}{|c|}{ Cycles Completed Low Density PMMA } & \multicolumn{5}{|c|}{ Stiffness (N/mm) Low Density PMMA } \\
\hline & \multicolumn{2}{|c|}{ Locking } & \multicolumn{2}{|c|}{ Nonlocking } & & \multicolumn{2}{|c|}{ Locking } & \multicolumn{2}{|c|}{ Nonlocking } \\
\hline & Large* & Stripped** & Large & Stripped & & Large* & Stripped** & Large & Stripped \\
\hline Mean & 4000 & 4000 & 3597.2 & 3220.6 & Mean & 302.17 & 152.77 & 62.07 & 24.23 \\
\hline stdev & 0 & 0 & 348.8 & 453.9 & Stdev & 86.23 & 26.18 & 45.92 & 9.18 \\
\hline \multirow[t]{3}{*}{$\%$ Fail } & 0 & 0 & 80 & 100 & & \multicolumn{2}{|c|}{ *excludes outlier (4) } & & \\
\hline & \multicolumn{2}{|c|}{ *excludes outlier (4) } & & & & \multicolumn{2}{|c|}{ **excluding outlier (5) } & & \\
\hline & \multicolumn{2}{|c|}{ **excluding outlier (5) } & & & & & & & \\
\hline
\end{tabular}

Figure 42: Adjusted Displacement at 100N load level (top left) Load Strength (top right) Cycles Survived (bottom left) and Stiffness (bottom right) for PMMA cement group, low density. The Displacement averages (top left) were normalized to a single load level of $100 \mathrm{~N}$ rather than only showing the displacement at failure across the board. Different factor combinations (tight locking vs stripped nonlocking) often resulted in screw samples failing in different load levels, which would give skewed results if portrayed all in the same table. 

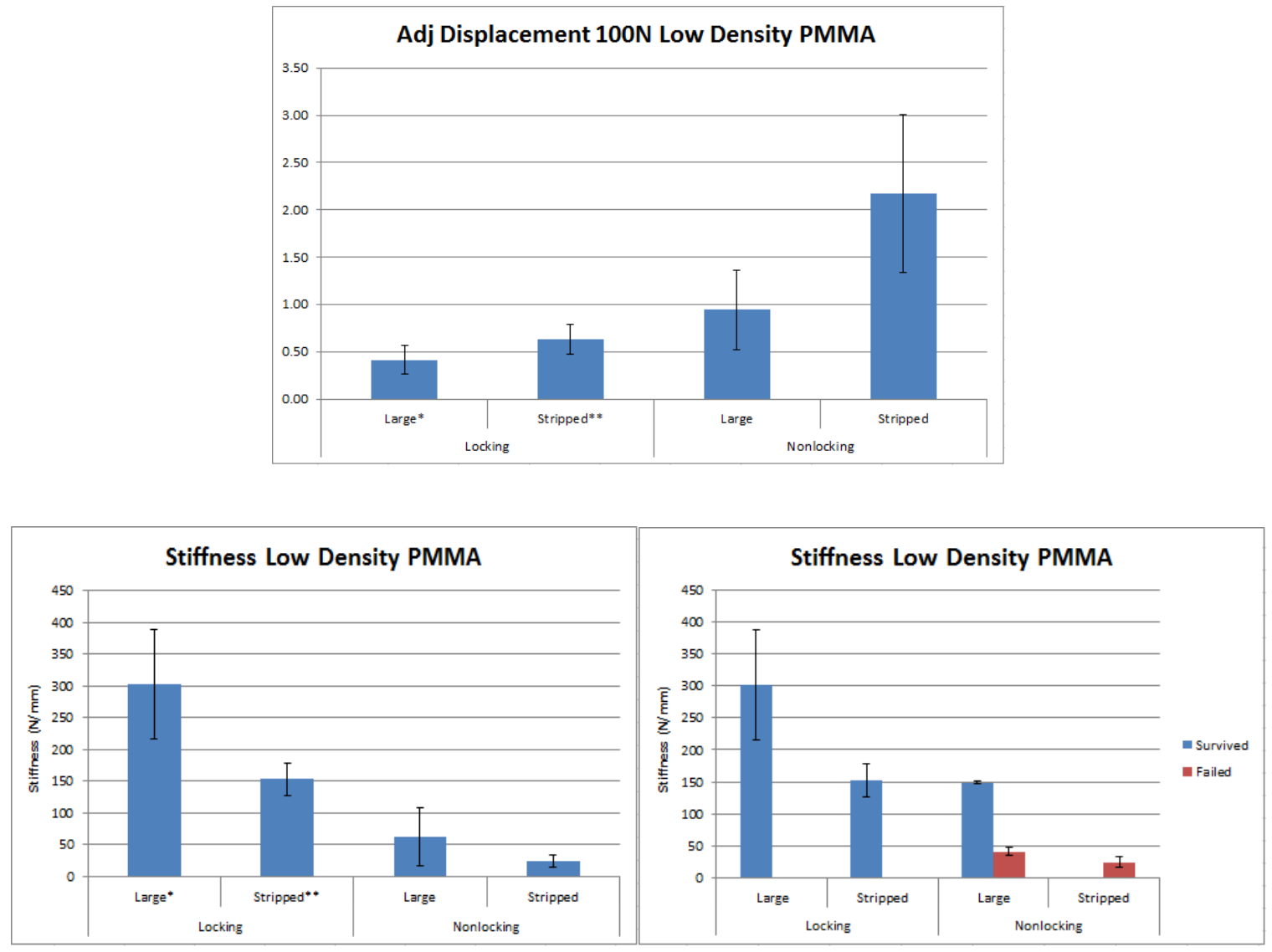

Figure 43: Adjusted Displacement averages at the 100N load level (top) and Stiffness (bottom row) PMMA cement group, low density. The bottom right graph shows the same Stiffness (bottom left) values but with sample survivals and failures separated for better visualization.

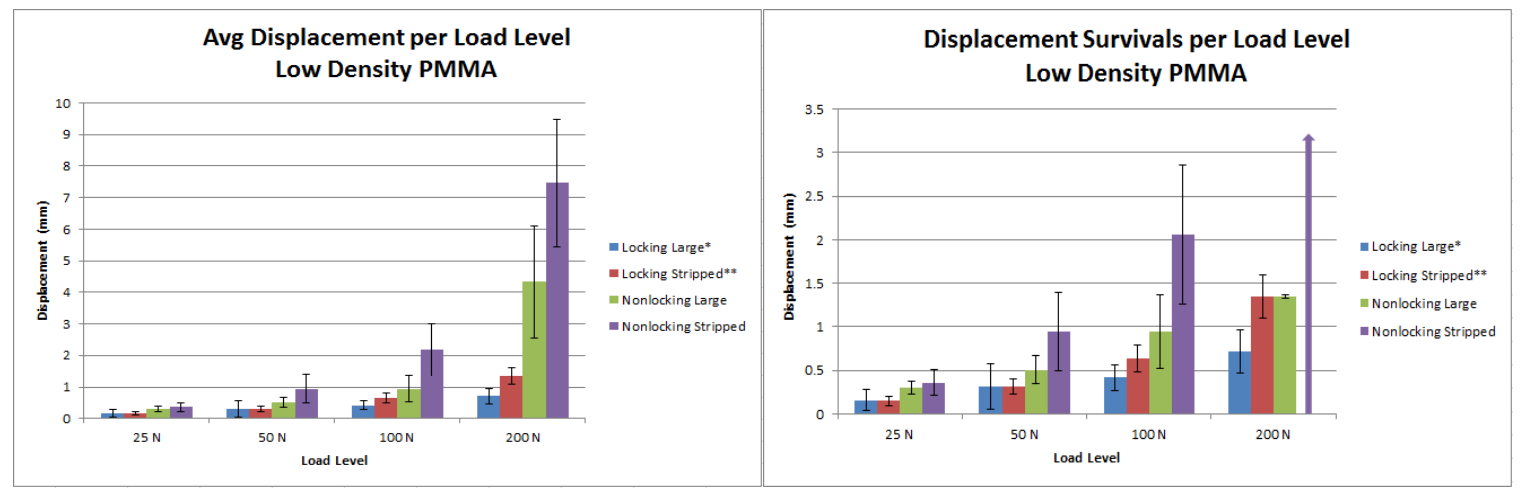

Figure 44: Adjusted Displacement per load level all samples (left) and Displacement screw survivals per load level (right) PMMA cement group low density. The right side graph shows displacements only for screws that survived the full 1000 cycles at the respective load level. The left side graph shows averaged displacements at each load level regardless of whether the sample failed at that load level or not. Arrows denote screw pullout before the current load level. 


\begin{tabular}{|c|c|c|c|c|c|c|c|c|c|}
\hline \multicolumn{5}{|c|}{ Adj Displacement (mm) 100N Medium Density PMMA } & \multicolumn{5}{|c|}{ Load Strength (N) Medium Density PMMA } \\
\hline & \multicolumn{2}{|c|}{ Locking } & \multicolumn{2}{|c|}{ Nonlocking } & & \multicolumn{2}{|c|}{ Locking } & \multicolumn{2}{|c|}{ Nonlocking } \\
\hline & Large & Stripped* & Large & Stripped ${ }^{* *}$ & & Large & Stripped* & Large & Stripped** \\
\hline mean & 0.25 & 0.47 & 0.55 & 2.20 & mean & 200.0 & 200.0 & 200.0 & 137.50 \\
\hline \multirow[t]{3}{*}{ stdev } & 0.07 & 0.12 & 0.04 & 0.99 & stdev & 0.0 & 0.0 & 0.0 & 69.44 \\
\hline & \multicolumn{2}{|c|}{ *excludes outlier (7) } & & & & \multicolumn{2}{|c|}{ * excludes outlier (7) } & & \\
\hline & \multicolumn{2}{|c|}{ **excludes outliers $(2,5)$} & & & & \multicolumn{2}{|c|}{ **excludes outliers $(2,5)$} & & \\
\hline
\end{tabular}

\begin{tabular}{|c|c|c|c|c|c|c|c|c|c|}
\hline \multicolumn{5}{|c|}{ Cycles Completed Medium Density PMMA } & \multicolumn{5}{|c|}{ Stiffness (N/mm) Medium Density PMMA } \\
\hline & \multicolumn{2}{|c|}{ Locking } & \multicolumn{2}{|c|}{ Nonlocking } & & \multicolumn{2}{|c|}{ Locking } & \multicolumn{2}{|c|}{ Nonlocking } \\
\hline & Large & Stripped* & Large & Stripped** & & Large & Stripped* & Large & Stripped ${ }^{* *}$ \\
\hline mean & 4000 & 4000 & 3853.70 & 2670.25 & mean & 639.64 & 274.94 & 108.73 & 33.17 \\
\hline stdev & 0 & 0 & 181.74 & 979.66 & stdev & 187.85 & 72.70 & 78.00 & 12.29 \\
\hline \multirow[t]{3}{*}{$\%$ Fail } & 0 & 0 & 60 & 87.5 & & \multicolumn{2}{|c|}{ * excludes outlier (7) } & & \\
\hline & \multicolumn{2}{|c|}{ * excludes outlier (7) } & & & & **excludes & $\operatorname{ers}(2,5)$ & & \\
\hline & \multicolumn{2}{|c|}{ **excludes outliers $(2,5)$} & & & & & & & \\
\hline
\end{tabular}

Figure 45: Adjusted Displacement at 100N load level (top left) Load Strength (top right) Cycles Survived (bottom left) and Stiffness (bottom right) for PMMA cement group, medium density. The Displacement averages (top left) were normalized to a single load level of $100 \mathrm{~N}$ rather than only showing the displacement at failure across the board. Different factor combinations (tight locking vs stripped nonlocking) often resulted in screw samples failing in different load levels, which would give skewed results if portrayed all in the same table.
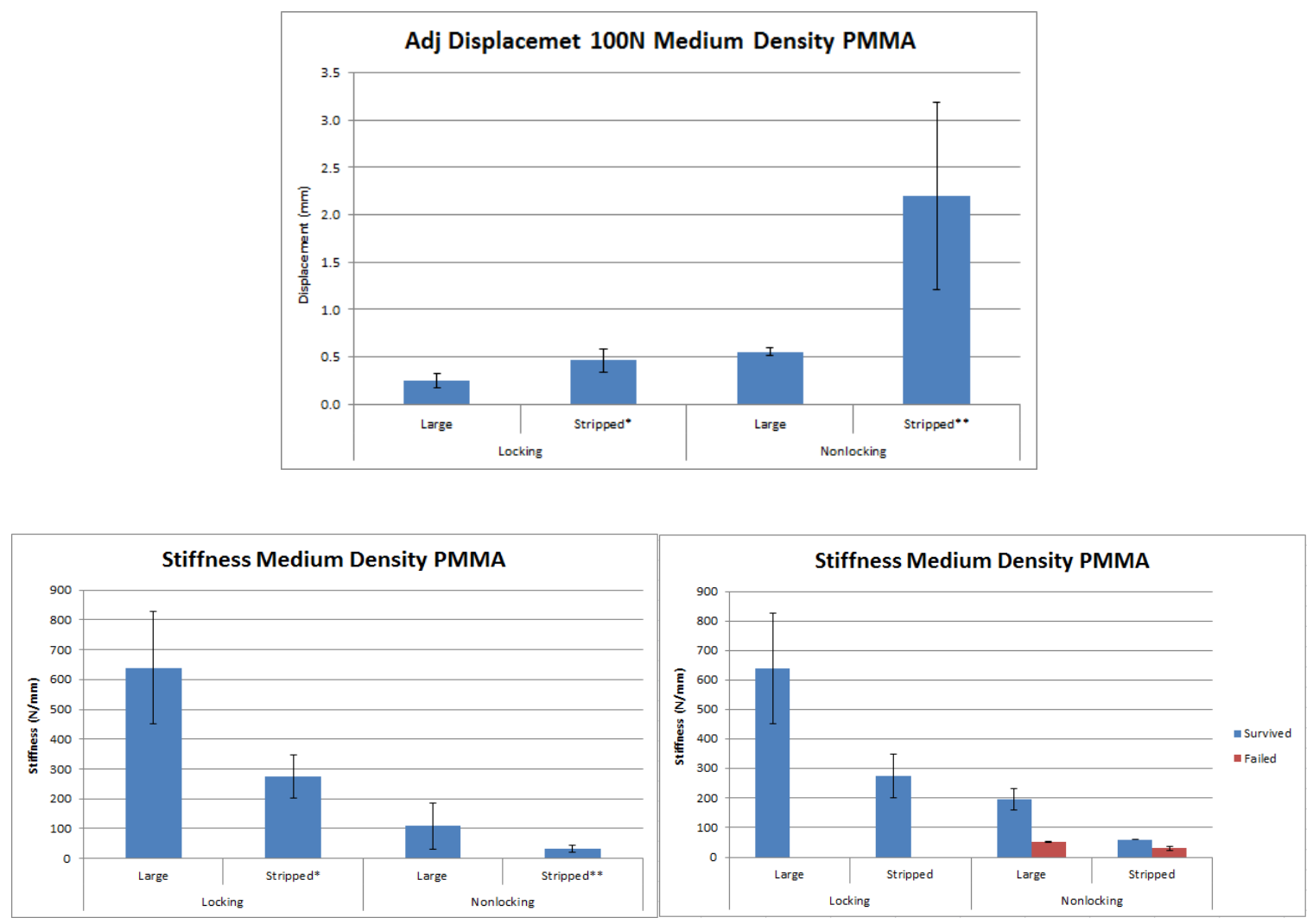

Figure 46: Adjusted Displacement averages at the $100 \mathrm{~N}$ load level (top) and Stiffness (bottom row) PMMA cement group, medium density. The bottom right graph shows the same Stiffness (bottom left) values but with sample survivals and failures separated for better visualization. 


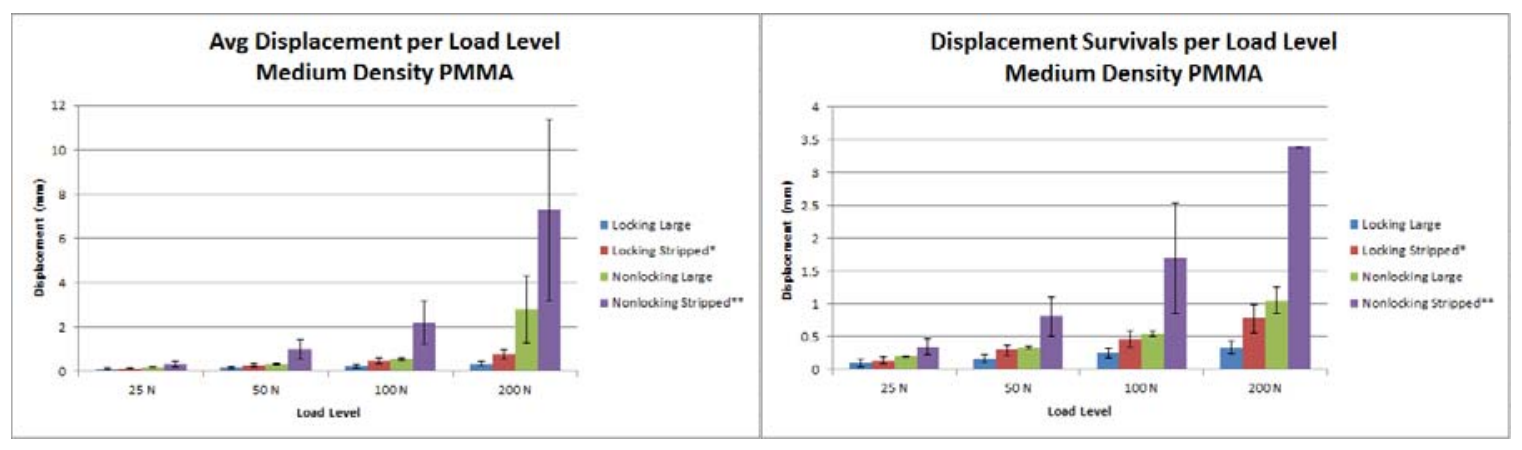

Figure 47: Adjusted Displacement per load level all samples (left) and Displacement screw survivals per load level (right) PMMA cement group medium density. The right side graph shows displacements only for screws that survived the full 1000 cycles at the respective load level. The left side graph shows averaged displacements at each load level regardless of whether the sample failed at that load level or not.

\begin{tabular}{|l|c|c|c|c|c|c|c|c|c|}
\hline \multicolumn{4}{|c|}{ Adj Displacement $(\mathbf{m m})$} & \multicolumn{4}{c|}{ 100N High Density PMMA } & \multicolumn{3}{c|}{ Load Strength (N) High Density PMMA } \\
\hline & \multicolumn{3}{|c|}{ Locking } & \multicolumn{2}{c|}{ Nonlocking } & & \multicolumn{2}{c|}{ Locking } & Nonlocking \\
\hline & Large & Stripped & Large & Stripped & & Large & Stripped & Large & Stripped \\
\hline mean & 0.31 & 0.40 & 0.55 & 1.04 & mean & 200.0 & 200.0 & 200.0 \\
\hline stdev & 0.14 & 0.08 & 0.09 & 0.35 & stdev & 0.0 & 0.0 & 0.0 \\
\hline
\end{tabular}

\begin{tabular}{|c|c|c|c|c|c|c|c|c|c|}
\hline \multicolumn{5}{|c|}{ Cycles Survived High Density PMMA } & \multicolumn{5}{|c|}{ Stiffness ( $\mathrm{N} / \mathrm{mm}$ ) High Density PMMA } \\
\hline & \multicolumn{2}{|c|}{ Locking } & \multicolumn{2}{|c|}{ Nonlocking } & & \multicolumn{2}{|c|}{ Locking } & \multicolumn{2}{|c|}{ Nonlocking } \\
\hline & Large & Stripped & Large & Stripped & & Large & Stripped & Large & Stripped \\
\hline mean & 4000 & 4000 & 3813.3 & 3538.9 & mean & 558.05 & 272.23 & 122.73 & 57.10 \\
\hline stdev & 0 & 0 & 258.9 & 332.9 & stdev & 158.03 & 53.82 & 79.73 & 36.14 \\
\hline$\%$ Fail & 0 & 0 & 50 & 70 & & & & & \\
\hline
\end{tabular}

Figure 48: Adjusted Displacement at 100N load level (top left) Load Strength (top right) Cycles Survived (bottom left) and Stiffness (bottom right) for PMMA cement group, high density. The Displacement averages (top left) were normalized to a single load level of $100 \mathrm{~N}$ rather than only showing the displacement at failure across the board. Different factor combinations (tight locking vs stripped nonlocking) often resulted in screw samples failing in different load levels, which would give skewed results if portrayed all in the same table. 

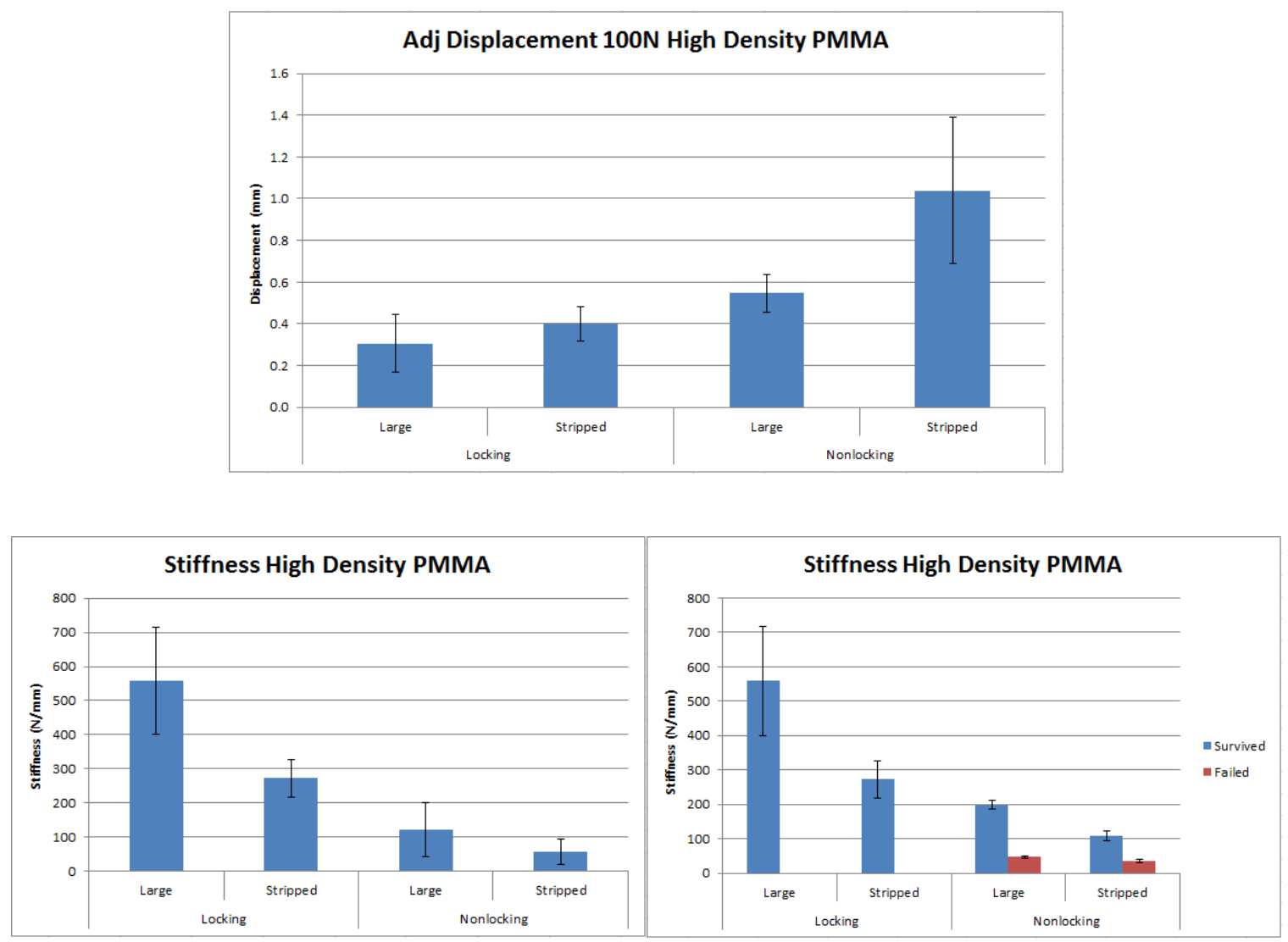

Figure 49: Adjusted Displacement averages at the 100N load level (top) and Stiffness (bottom row) PMMA cement group, high density. The bottom right graph shows the same Stiffness (bottom left) values but with sample survivals and failures separated for better visualization.

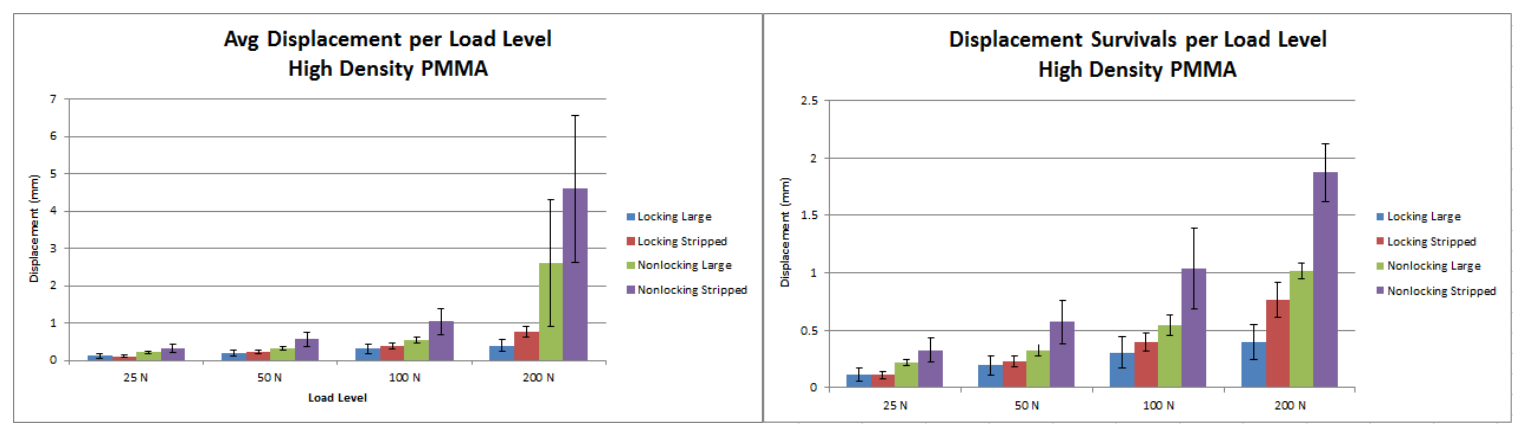

Figure 50: Adjusted Displacement per load level all samples (left) and Displacement screw survivals per load level (right) PMMA cement group high density. The right side graph shows displacements only for screws that survived the full 1000 cycles at the respective load level. The left side graph shows averaged displacements at each load level regardless of whether the sample failed at that load level or not. 


\section{CaP Cement Group}

\begin{tabular}{|c|c|c|c|c|c|c|c|c|c|}
\hline \multicolumn{5}{|c|}{ Adj Displacement (mm) 100N Low Density CaP } & \multicolumn{5}{|c|}{ Load Strength (N) Low Density CaP } \\
\hline & \multicolumn{2}{|c|}{ Locking } & \multicolumn{2}{|c|}{ Nonlocking } & & \multicolumn{2}{|c|}{ Locking } & \multicolumn{2}{|c|}{ Nonlocking } \\
\hline & Large & Stripped & Large & Stripped* & & Large & Stripped & Large & Stripped* \\
\hline mean & 0.64 & 1.20 & 1.99 & 7.62 & mean & 200.0 & 180.0 & 200.0 & 55.56 \\
\hline \multirow[t]{2}{*}{ stdev } & 0.14 & 0.43 & 0.85 & 1.56 & stdev & 0.0 & 42.16 & 0.0 & 27.32 \\
\hline & \multicolumn{2}{|c|}{ *excludes outlier (1) } & & & & \multicolumn{2}{|c|}{ *excludes outlier (1) } & & \\
\hline
\end{tabular}

\begin{tabular}{|c|c|c|c|c|c|c|c|c|c|}
\hline \multicolumn{5}{|c|}{ Cycles Completed Low Density CaP } & \multicolumn{5}{|c|}{ Stiffness (N/mm) Low Density CaP } \\
\hline & \multicolumn{2}{|c|}{ Locking } & \multicolumn{2}{|c|}{ Nonlocking } & & \multicolumn{2}{|c|}{ Locking } & \multicolumn{2}{|c|}{ Nonlocking } \\
\hline & Large & Stripped & Large & Stripped* & & Large & Stripped & Large & Stripped* \\
\hline Mean & 3910.5 & 3184.1 & 3182.9 & 1435.6 & mean & 146.52 & 54.79 & 25.75 & 14.62 \\
\hline Stdev & 210.6 & 572.3 & 98.3 & 698.5 & stdev & 55.17 & 10.55 & 3.58 & 2.64 \\
\hline \multirow[t]{2}{*}{$\%$ Fail } & 20 & 100 & 100 & 100 & & *excludes & tlier (1) & & \\
\hline & \multicolumn{2}{|c|}{ *excludes outlier (1) } & & & & & & & \\
\hline
\end{tabular}

Figure 51: Adjusted Displacement at 100N load level (top left) Load Strength (top right) Cycles Survived (bottom left) and Stiffness (bottom right) for Calcium phosphate cement group, low density. The Displacement averages (top left) were normalized to a single load level of $100 \mathrm{~N}$ rather than only showing the displacement at failure across the board. Different factor combinations (tight locking vs stripped nonlocking) often resulted in screw samples failing in different load levels, which would give skewed results if portrayed all in the same table. 


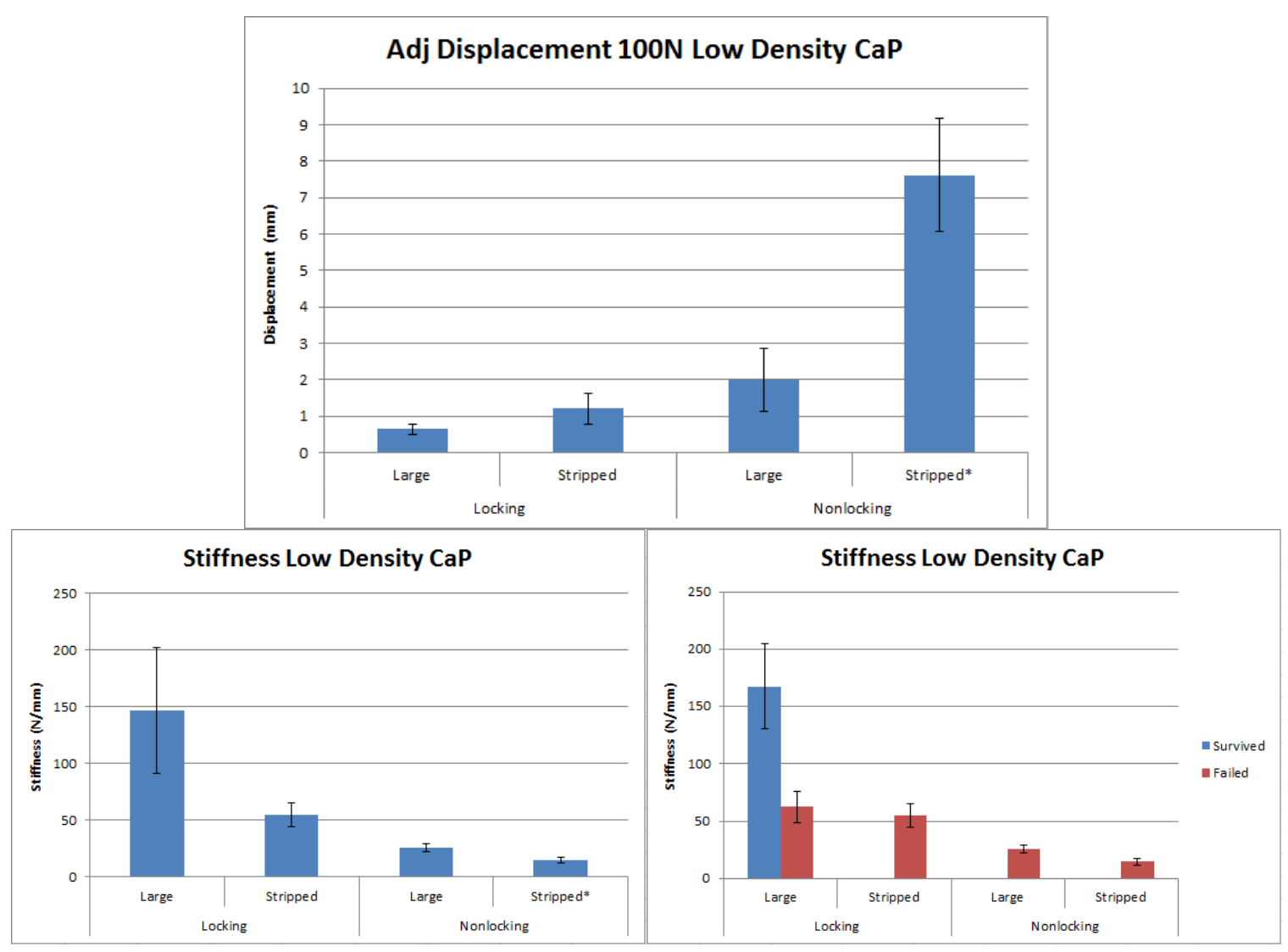

Figure 52: Adjusted Displacement averages at the 100N load level (top) and Stiffness (bottom row) Calcium phosphate cement group, low density. The bottom right graph shows the same Stiffness (bottom left) values but with sample survivals and failures separated for better visualization.

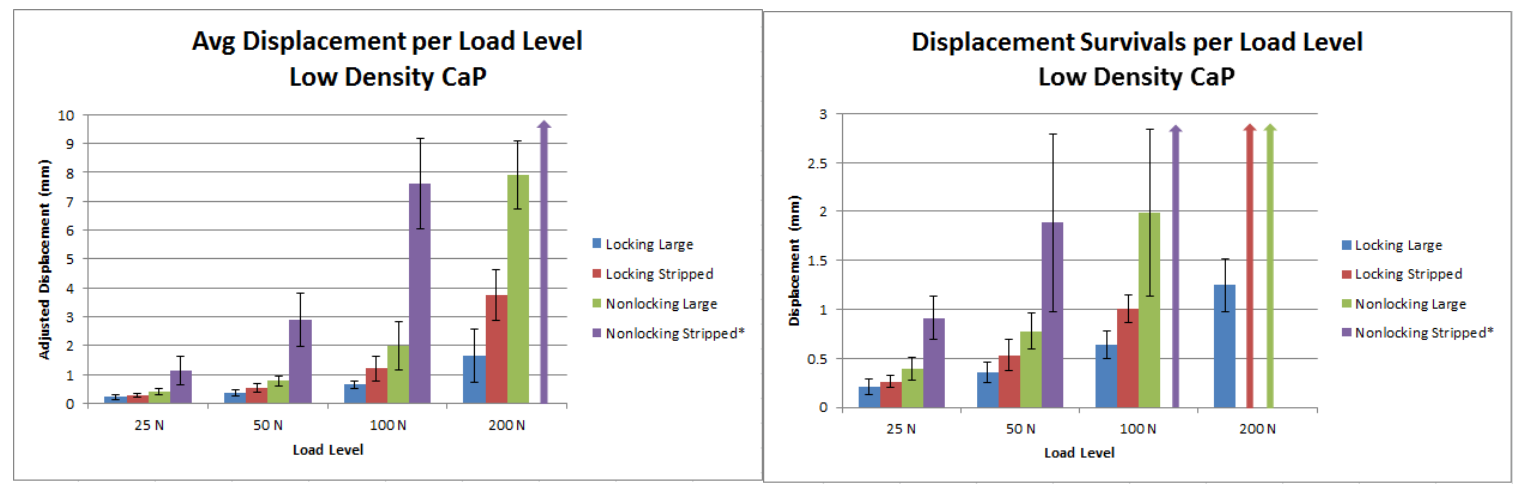

Figure 53: Adjusted Displacement per load level all samples (left) and Displacement screw survivals per load level (right) Calcium phosphate cement group, low density. The right side graph shows displacements only for screws that survived the full 1000 cycles at the respective load level. The left side graph shows averaged displacements at each load level regardless of whether the sample failed at that load level or not. Arrows denote screw pullout before the current load level. 


\begin{tabular}{|c|c|c|c|c|c|c|c|c|c|}
\hline \multicolumn{5}{|c|}{ Adj Displacement (mm) 100N Medium Density CaP } & \multicolumn{5}{|c|}{ Load Strength $(\mathrm{N})$ Medium Density CaP } \\
\hline & \multicolumn{2}{|c|}{ Locking } & \multicolumn{2}{|c|}{ Nonlocking } & & \multicolumn{2}{|c|}{ Locking } & \multicolumn{2}{|c|}{ Nonlocking } \\
\hline & Large & Stripped* & Large & Stripped & & Large & Stripped* & Large & Stripped \\
\hline mean & 0.57 & 0.93 & 1.06 & 1.89 & mean & 200.0 & 200.0 & 200.0 & 180.0 \\
\hline \multirow[t]{2}{*}{ stdev } & 0.25 & 0.23 & 0.09 & 0.89 & stdev & 0.0 & 0.0 & 0.0 & 42.16 \\
\hline & \multicolumn{2}{|c|}{ *excludes outlier (1) } & & & & \multicolumn{2}{|c|}{ *excluding outlier (1) } & & \\
\hline
\end{tabular}

\begin{tabular}{|c|c|c|c|c|c|c|c|c|c|}
\hline \multicolumn{5}{|c|}{ Cycles Completed Medium Density CaP } & \multicolumn{5}{|c|}{ Stiffness (N/mm) Medium Density CaP } \\
\hline & \multicolumn{2}{|c|}{ Locking } & \multicolumn{2}{|c|}{ Nonlocking } & & \multicolumn{2}{|c|}{ Locking } & \multicolumn{2}{|c|}{ Nonlocking } \\
\hline & Large & Stripped* & Large & Stripped & & Large & Stripped* & Large & Stripped \\
\hline Mean & 3983 & 3623.89 & 3165.17 & 2992.10 & mean & 187.85 & 76.40 & 29.34 & 27.04 \\
\hline Stdev & 53.76 & 413.94 & 42.29 & 230.54 & stdev & 76.75 & 17.15 & 2.76 & 1.66 \\
\hline \multirow[t]{2}{*}{$\%$ Fail } & 10 & 66.67 & 100 & 100 & & \multicolumn{2}{|c|}{ *excluding outlier (1) } & & \\
\hline & \multicolumn{2}{|c|}{ *excluding outlier (1) } & & & & & & & \\
\hline
\end{tabular}

Figure 54: Adjusted Displacement at 100N load level (top left) Load Strength (top right) Cycles Survived (bottom left) and Stiffness (bottom right) for Calcium phosphate cement group, medium density. The Displacement averages (top left) were normalized to a single load level of $100 \mathrm{~N}$ rather than only showing the displacement at failure across the board. Different factor combinations (tight locking vs stripped nonlocking) often resulted in screw samples failing in different load levels, which would give skewed results if portrayed all in the same table.

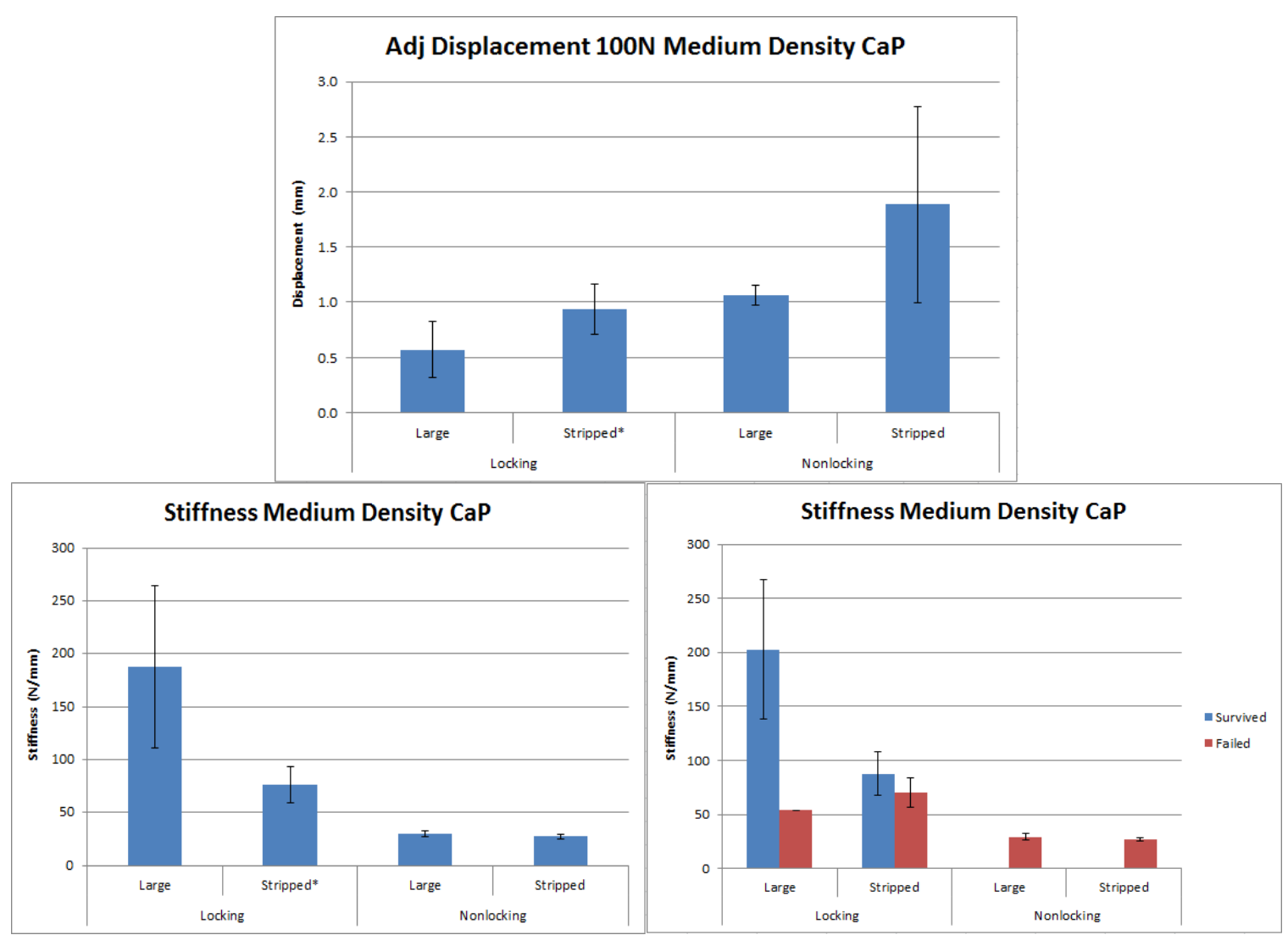

Figure 55: Adjusted Displacement averages at the $100 \mathrm{~N}$ load level (top) and Stiffness (bottom row) Calcium phosphate cement group, medium density. The bottom right graph shows the same Stiffness (bottom left) values but with sample survivals and failures separated for better visualization. 


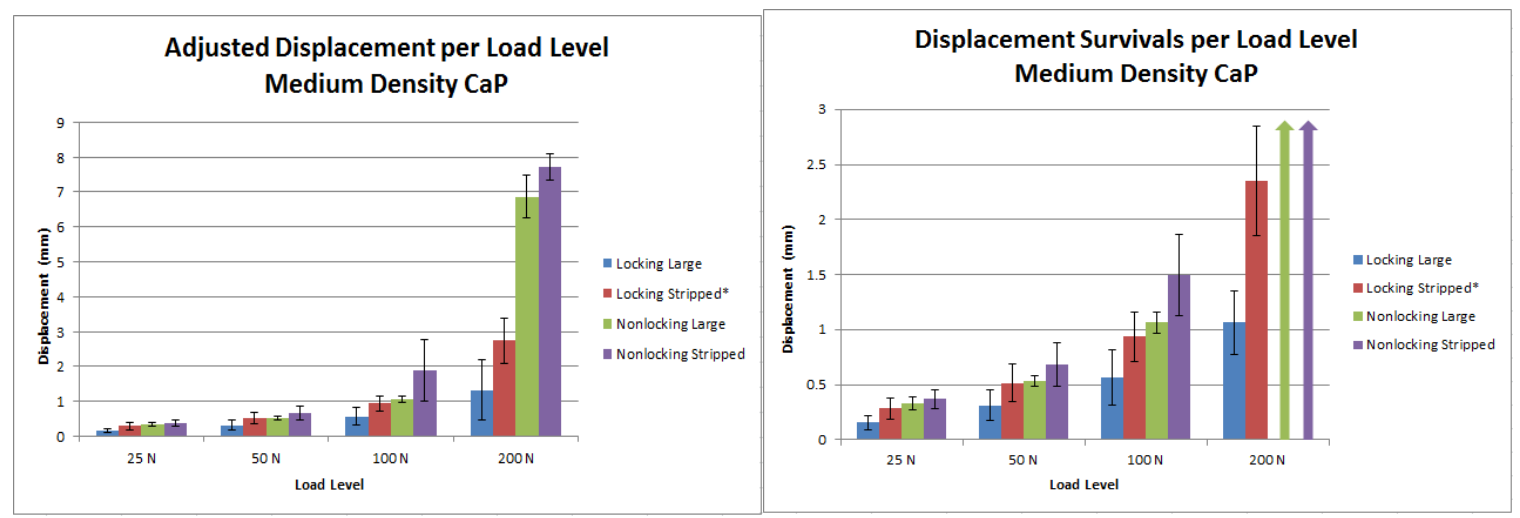

Figure 56: Adjusted Displacement per load level all samples (left) and Displacement screw survivals per load level (right) Calcium phosphate cement group, medium density. The right side graph shows displacements only for screws that survived the full 1000 cycles at the respective load level. The left side graph shows averaged displacements at each load level regardless of whether the sample failed at that load level or not. Arrows denote screw pullout before the current load level.

\begin{tabular}{|c|c|c|c|c|c|c|c|c|c|}
\hline \multicolumn{5}{|c|}{ Adj Displacement (mm) 100N High Density CaP } & \multicolumn{5}{|c|}{ Load Strength (N) High Density CaP } \\
\hline & \multicolumn{2}{|c|}{ Locking } & \multicolumn{2}{|c|}{ Nonlocking } & & \multicolumn{2}{|c|}{ Locking } & \multicolumn{2}{|c|}{ Nonlocking } \\
\hline & Large & Stripped & Large & Stripped* & & Large & Stripped & Large & Stripped* \\
\hline mean & 0.91 & 1.40 & 2.95 & 4.28 & mean & 166.67 & 170.0 & 166.67 & 125.0 \\
\hline \multirow[t]{2}{*}{ stdev } & 0.65 & 1.35 & 2.83 & 3.13 & stdev & 57.74 & 48.30 & 57.74 & 65.47 \\
\hline & \multicolumn{2}{|c|}{ *excludes outliers $(6,9)$} & & & & \multicolumn{2}{|c|}{ *excludes outliers $(6,9)$} & & \\
\hline
\end{tabular}

\begin{tabular}{|c|c|c|c|c|c|c|c|c|c|}
\hline \multicolumn{5}{|c|}{ Cycles Completed High Density CaP } & \multicolumn{5}{|c|}{ Stiffness (N/mm) High Density CaP } \\
\hline & \multicolumn{2}{|c|}{ Locking } & \multicolumn{2}{|c|}{ Nonlocking } & & \multicolumn{2}{|c|}{ Locking } & \multicolumn{2}{|c|}{ Nonlocking } \\
\hline & Large & Stripped & Large & Stripped* & & Large & Stripped & Large & Stripped* \\
\hline Mean & 2971 & 3206 & 2791 & 2355.13 & mean & 50.06 & 123.69 & 22.81 & 19.87 \\
\hline Stdev & 327.62 & 648.20 & 573.31 & 843.15 & stdev & 8.83 & 134.23 & 5.97 & 8.86 \\
\hline \multirow[t]{2}{*}{$\%$ Fail } & 100 & 70 & 100 & 100 & & *excludes o & $s(6,9)$ & & \\
\hline & \multicolumn{2}{|c|}{ *excludes outliers $(6,9)$} & & & & & & & \\
\hline
\end{tabular}

Figure 57: Adjusted Displacement at 100N load level (top left) Load Strength (top right) Cycles Survived (bottom left) and Stiffness (bottom right) for Calcium phosphate cement group, high density. The Displacement averages (top left) were normalized to a single load level of $100 \mathrm{~N}$ rather than only showing the displacement at failure across the board. Different factor combinations (tight locking vs stripped nonlocking) often resulted in screw samples failing in different load levels, which would give skewed results if portrayed all in the same table. 

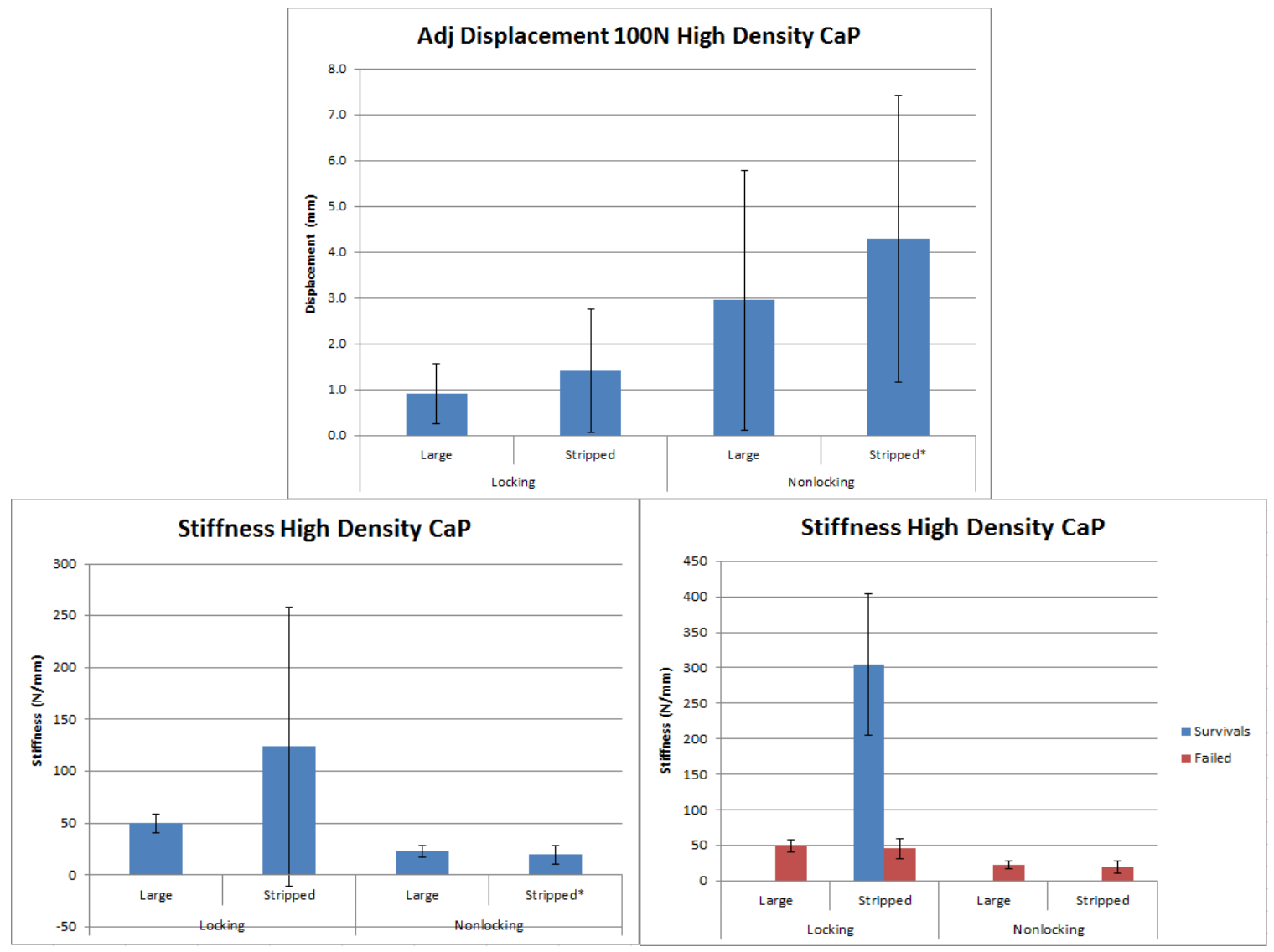

Figure 58: Adjusted Displacement averages at the 100N load level (top) and Stiffness (bottom row) Calcium phosphate cement group, high density. The bottom right graph shows the same Stiffness (bottom left) values but with sample survivals and failures separated for better visualization. The abnormally large amount of standard deviation shown for the stripped locking samples (bottom left) was due to the large difference in stiffness values between samples that survived the test compared to samples that failed (bottom right).

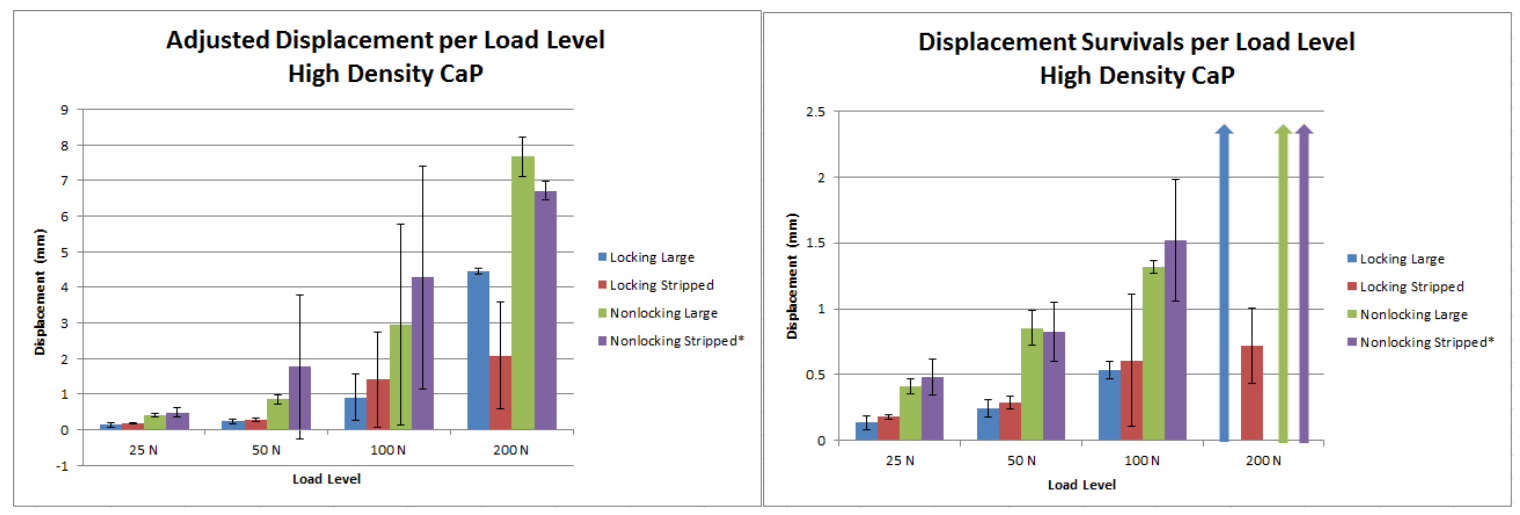

Figure 59: Adjusted Displacement per load level all samples (left) and Displacement screw survivals per load level (right) Calcium phosphate cement group, high density. The right side graph shows displacements only for screws that survived the full 1000 cycles at the respective load level. The left side graph shows averaged displacements at each load level regardless of whether the sample failed at that load level or not. Arrows denote screw pullout before the current load level. 


\section{Comprehensive Results}

The original objective of this study was to directly compare the cyclic and pullout strength of the Calcium Phosphate cement to the presumably stronger PMMA cement. The lowest density foam blocks in this study closely resembled the density of osteoporotic bone and therefore the most relevant density for this comparison.

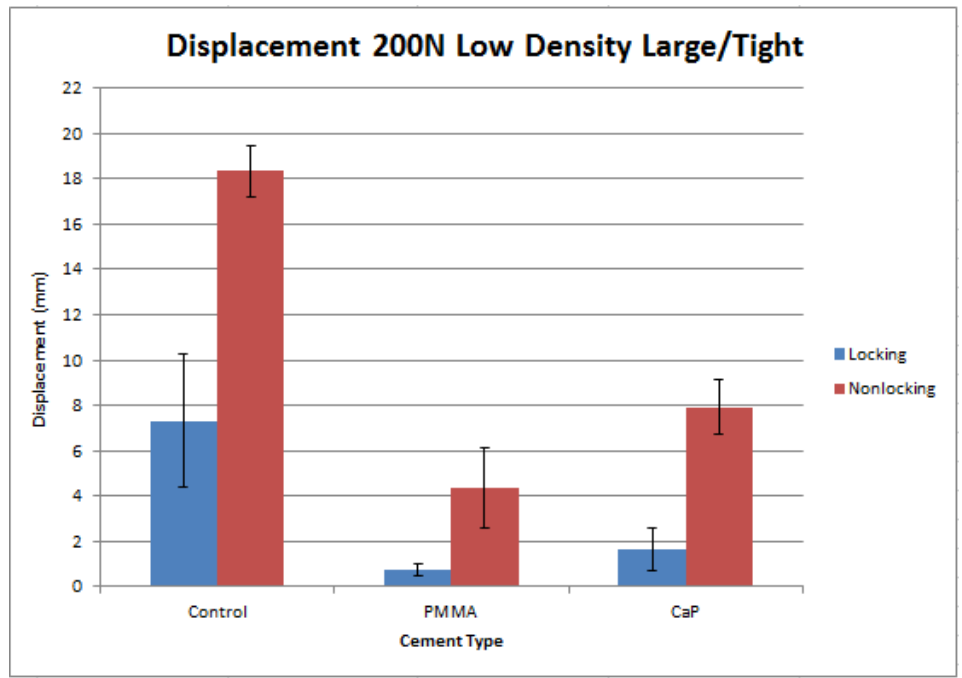

Figure 60: Adjusted Displacement at 200N load level low density foam blocks for control group and both PMMA and CaP cement, non-stripped holes. The "tight" holes in the control group duplicate the "large" holes in cement groups in that the screws were either encompassed completely by cement or surrounding bone mass.

Both PMMA cement and CaP cement greatly reduced the amount of displacement at the $200 \mathrm{~N}$ load level compared to the control group for non-stripped holes. This was to be expected, especially with the "low" density foam bone block $\left(12.5 \mathrm{lbs} / \mathrm{ft}^{3}\right)$. Polymethyl Methacrylate cement has previously been proven to be an extremely durable adhesive in terms of direct pullout strength, but the novel cyclic test setup in this study has appeared to level the playing field, at least for osteoporotic-like bone. 


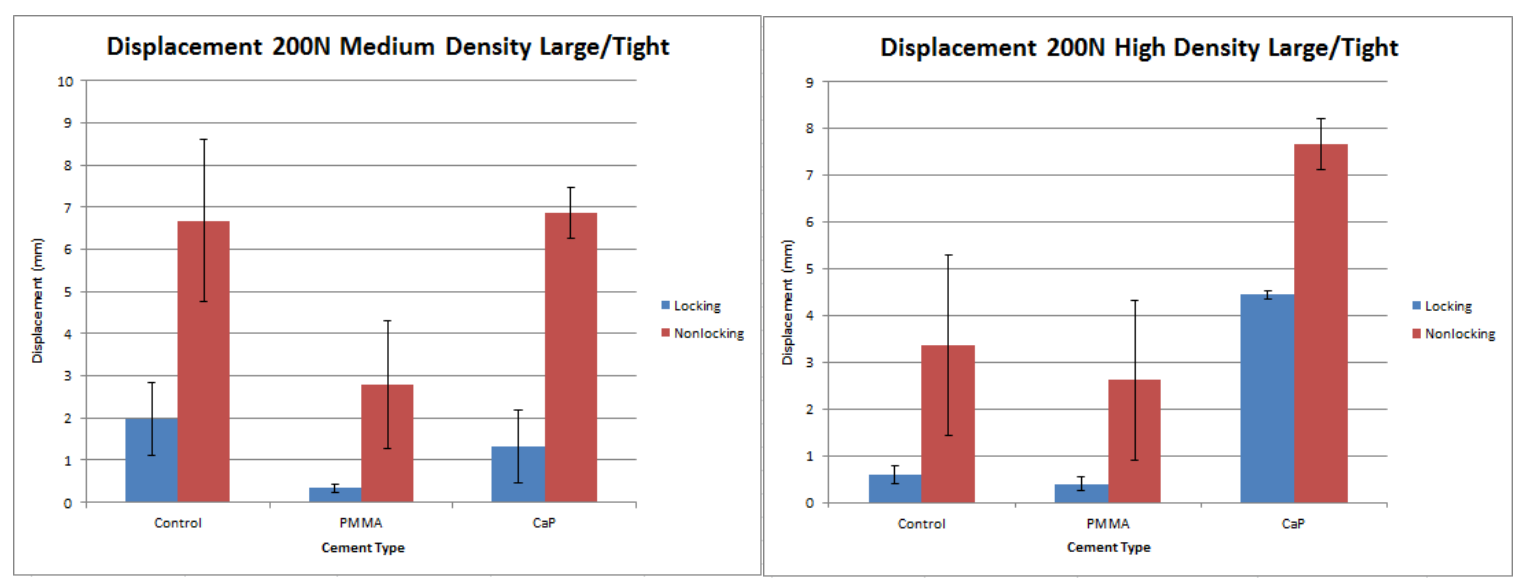

Figure 61: Adjusted Displacement at 200N load level for control group, PMMA cement and CaP cement at medium (left) and high (right) density foam samples, non-stripped holes. The "tight" holes in the control group duplicate the "large" holes in cement groups in that the screws were either encompassed completely by cement or surrounding bone mass.

The results for the medium and high density foam blocks tell a different story of the cement groups displacement compared to the control group for large or tight screw holes. In the medium density, Figure 61 (above left), calcium phosphate showed very similar results to the control group while the PMMA cement was noticeably less compliant for both locking and nonlocking screw fixation types. And in the highest density foam blocks, Figure 61 (above right), the control group and PMMA cement group were very similar while calcium phosphate showed very little resistance to movement and much more adjusted displacement averages. It was truly a tale of three densities for calcium phosphate displacement results. 


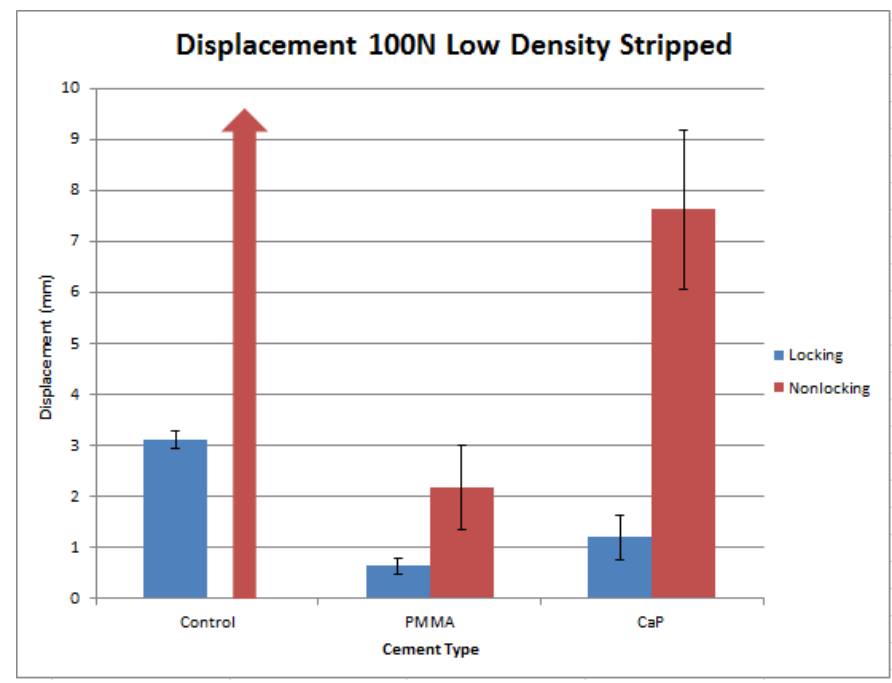

Figure 62: Adjusted Displacement at 100N load level low density control group, PMMA cement and $\mathrm{CaP}$ cement groups, stripped screw holes. The large arrow denotes complete screw pullout before the $100 \mathrm{~N}$ load level for designated sample group.

Stripped hole samples were more difficult to analyze because there was much more variability between samples. On first glance it appears that calcium phosphate didn't improve much on the control samples for low density bone and was nowhere close to PMMA. Yet the stripped nonlocking control samples didn't survive more than a few cycles each, let alone enough to record data for the $100 \mathrm{~N}$ load level. Granted most of the $\mathrm{CaP}$ stripped low nonlocking samples failed at or before the $100 \mathrm{~N}$ load level $(80 \%)$ it was still a vast improvement on the control group. Also, stripped hole low density samples with the locking plate for PMMA and CaP were within $1 \mathrm{~mm}$ of each other on average (PMMA: 0.64mm, CaP: 1.20mm). 


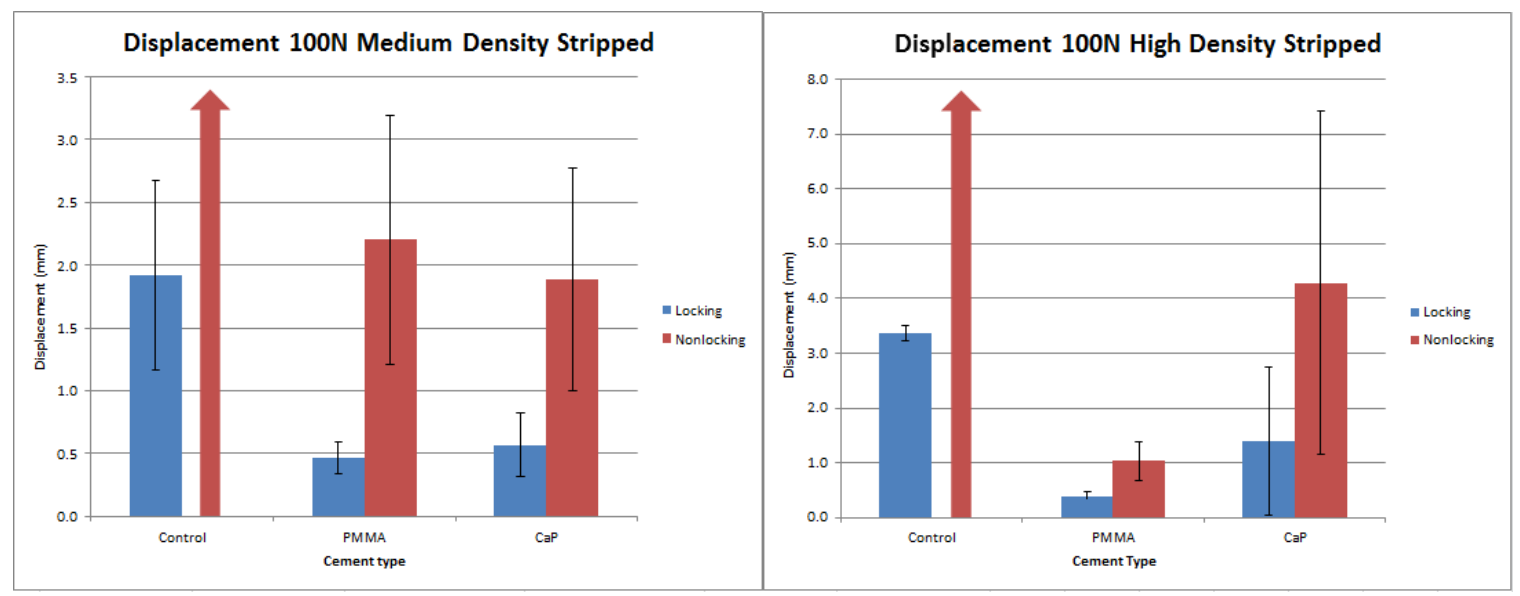

Figure 63: Adjusted Displacement at 100N load level for control group, PMMA cement and CaP cement at medium (left) and high (right) density bone blocks, stripped screw holes. Long arrows denote screw pullout before $100 \mathrm{~N}$ load level for designated sample groups.

A common trend in the stripped hole data was the nonlocking screws in the control group pulling out quickly. This made sense intuitively simply because the control group stripped samples were screws resting in cylindrical tunnels that were just slightly smaller than themselves with no cement to keep them stable. Locking stripped screws survived longer because the locking plate keeps the screws rigid which allowed at least a few threads to hug the inside wall at all times, but stripped control group samples with the nonlocking plate never lasted more than 4-5 cycles. The nonlocking plate allowed the screw to toggle and almost immediately find the correct angle for instant pullout.

Another trend for stripped holes in this study was the large variability between samples which causes the standard deviation to be almost as large as the averages in some cases. This was partly due to the unpredictability of the cement distribution in such a confined space. Generally there was only enough room for the cement between individual threads of the screws as the edge of the threads was (ideally) just touching the inside wall of the tunnel. With the deviations as high as they were, one would have to assume that the cement wasn't always distributed evenly within each stripped hole. 
Unfortunately, there was no practical way to find out for sure. The large variability was also due in part to the phenomenon of the calcium phosphate cement in high density foam blocks, which is described in detail below.

Displacement was arguably the most important response to consider for this study, but sometimes it didn't tell the whole story. The displacement values shown in the graphs above were all normalized to a single load level of $200 \mathrm{~N}$, but samples that did not last until the $200 \mathrm{~N}$ load level were excluded from those averages. The Stiffness response used all values by normalizing each one with its own load strength at failure so no samples were omitted from the results.

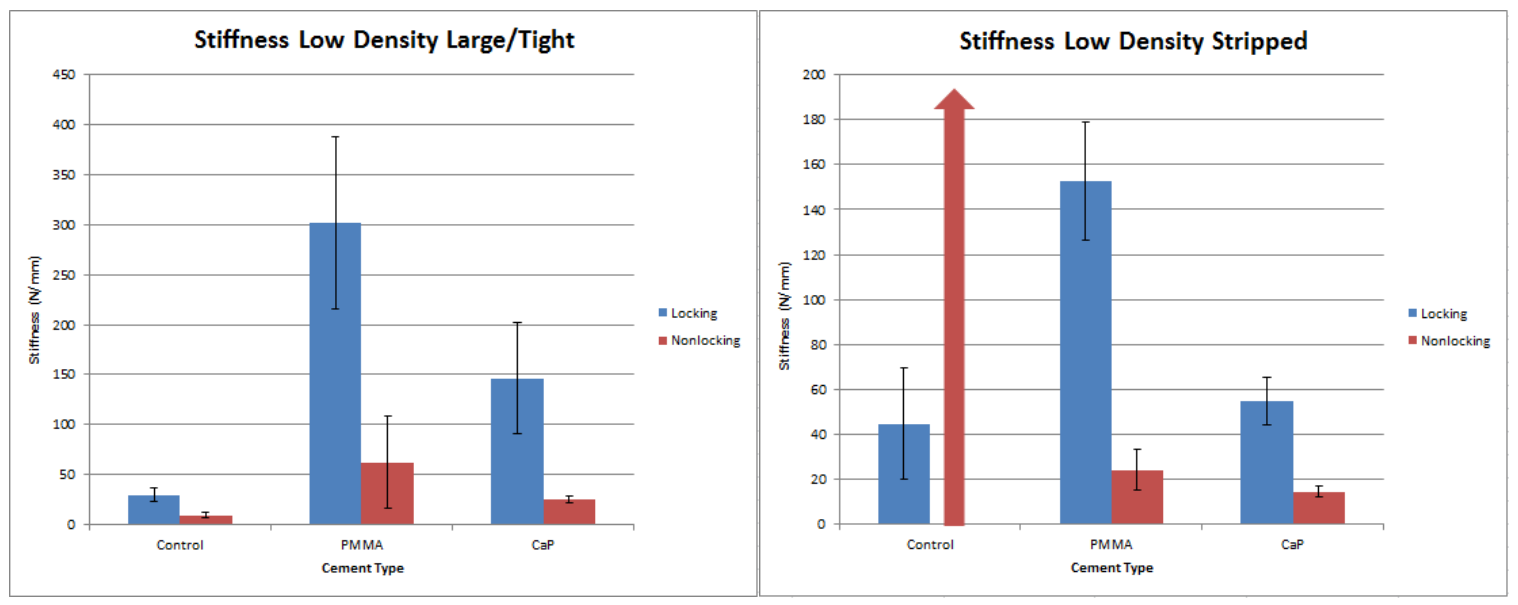

Figure 64: Stiffness results comparisons for control group, PMMA cement and CaP cement groups, large/tight (left) and stripped (right) for low density bone blocks. The "tight" holes in the control group duplicate the "large" holes in cement groups in that the screws were either encompassed completely by cement or surrounding bone mass. The arrow denotes immediate screw pullout for designated samples. The nonlocking stripped hole screws in the control group only lasted a cycle or two before completely pulling out of the foam which was enough to get an estimate on the displacement, but this value, along with the resulting stiffness calculation would not be accurate for comparisons with samples that lasted more than a few seconds.

Figure 64 above shows all stiffness results for large (tight) and stripped hole samples. Again, the large hole results show the most promise of calcium phosphate 
cement being comparable to the PMMA cement, as both cement groups have noticeable improvement over the control group. 4mm stripped holes indicate that PMMA was the dominant choice while $\mathrm{CaP}$ was much more similar to the control group, although the nonlocking samples were an obvious improvement from pulling out within a few seconds.
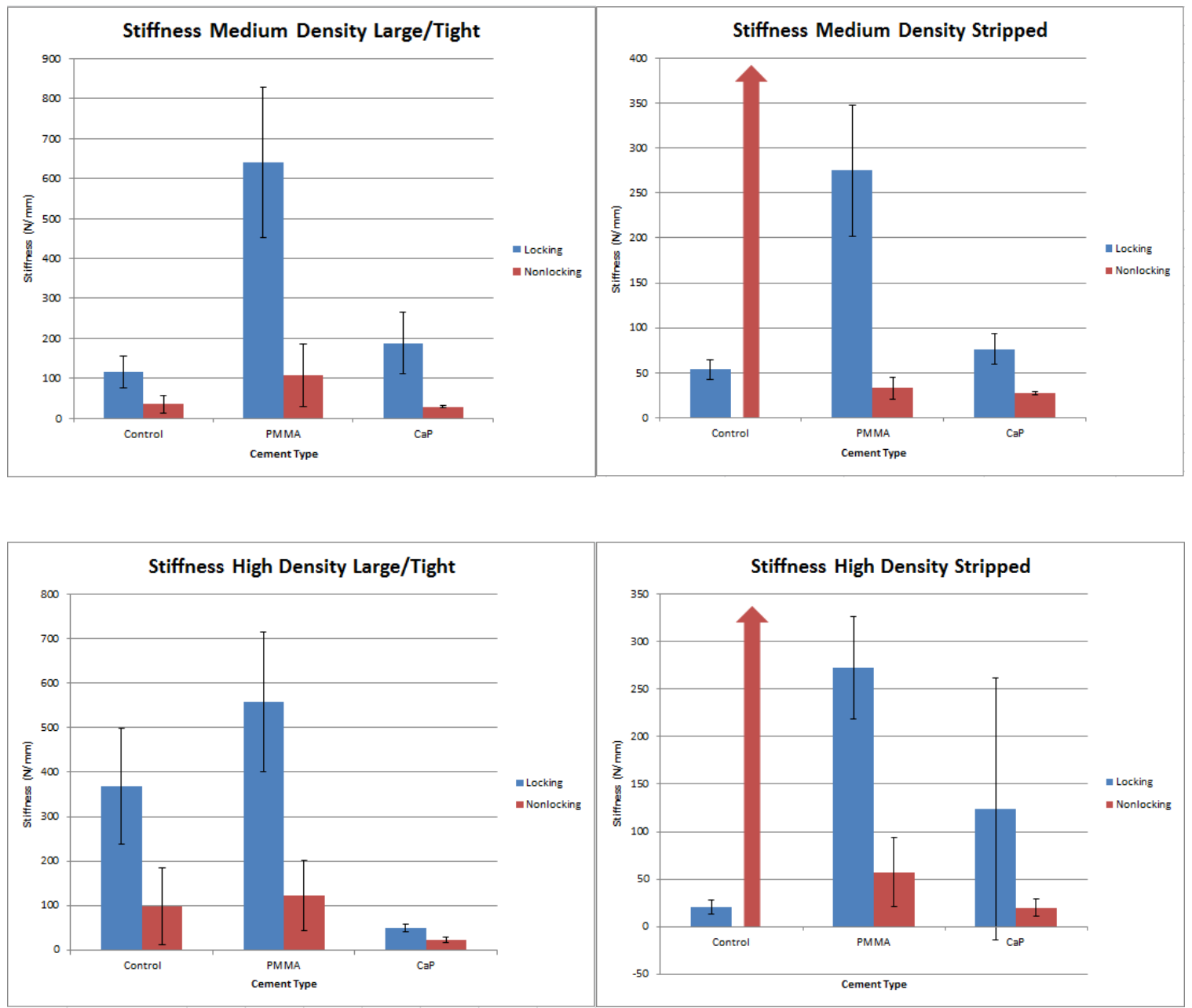

Figure 65: Stiffness results comparisons for control group, PMMA cement and CaP cement groups. The top row is medium density bone block samples, the bottom row is high density bone block samples and the left column shows large or tight screw holes while the right column is stripped screw holes. The "tight" holes in the control group duplicate the "large" holes in cement groups in that the screws were either encompassed completely by cement or surrounding bone mass. Arrows denote immediate screw pullout for designated samples. 
Medium and high density bone blocks, both shown in figure 65 above for large hole and stripped hole samples, again indicate PMMA as the clear cut favorite in maximizing screw stiffness. Calcium phosphate showed improvement over the control group with nonlocking stripped samples but they were relatively equal across the board in medium density bone blocks. The high density samples portrayed a concerning decrease in stiffness for calcium phosphate cement which was due to the interesting phenomenon described below. PMMA was assumed to be the strongest bone cement type prior to testing for this study so it was no surprise that it showed much higher stiffness values than the control group and $\mathrm{CaP}$ cement group across all densities and hole sizes. Calcium phosphate cement displayed very similar if not improved results to the control group in medium or "average" bone density. This fact in itself may be another important takeaway from this study. If $\mathrm{CaP}$ was demonstrated to be statistically equal to or slightly stronger than average human bone, it can be utilized in many different applications. However, further studies will be needed to substantiate the extended use of calcium phosphate.

The third and final major response to consider was the number of cycles the screw survived before pulling out of the block. This response was limited to a whole number between 1 and 4000 and was often maxed out by entire sections surviving the entire test. 

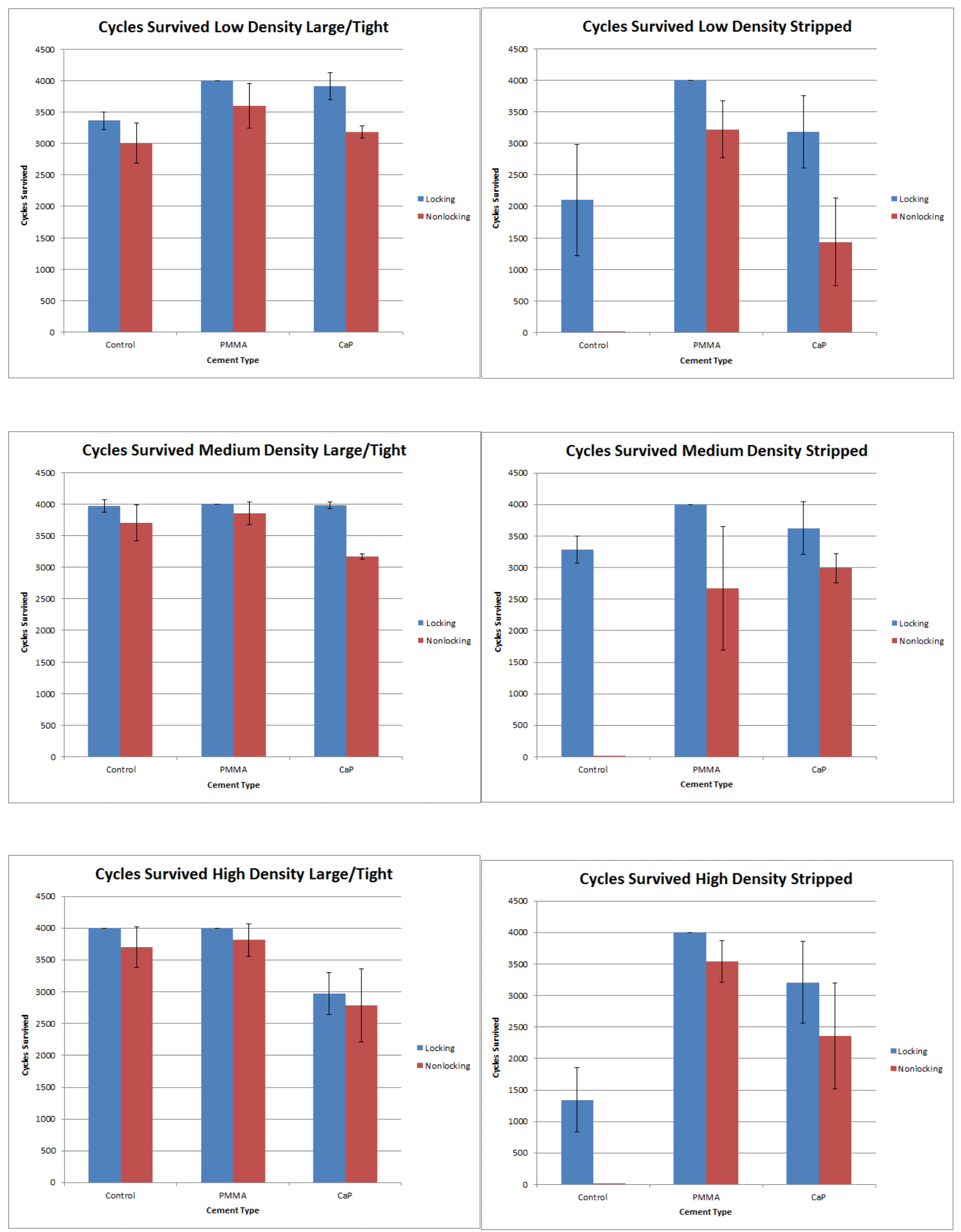

Figure 66: Cycles survived averages for control group, PMMA cement and $\mathrm{CaP}$ cement groups. Top row: low density. Middle row: medium density. Bottom row: high density. Large/tight hole size sample are found in the left column, and stripped screw hole sizes are shown in the right column above. The "tight" holes in the control group duplicate the "large" holes in cement groups in that the screws were either encompassed completely by cement or surrounding bone mass. 
Cycles survived averages were fairly consistent throughout, save for nonlocking stripped samples in the control group, as expected. The only noteworthy differences were within the low density stripped screw hole group (Figure 66 above, top row, right side). Both locking and nonlocking screws in the cement groups showed a distinguishable increase in cycles survived compared to the control group. The high density group (bottom row, right) also showed a distinct increase in cycles survived but the $\mathrm{CaP}$ samples in that density group exhibited the rare pull-out method described below which makes them incomparable.

\section{E. Post Hoc Comparisons}

Now that the main responses had been visualized between cement groups it was important to analyze the relevant statistical comparisons. The most pertinent factors appear to be the low density non-stripped groups (Figures 60 and 64 above).

Displacement at the $100 \mathrm{~N}$ and $200 \mathrm{~N}$ load level and Stiffness were the applicable factors for this case. 

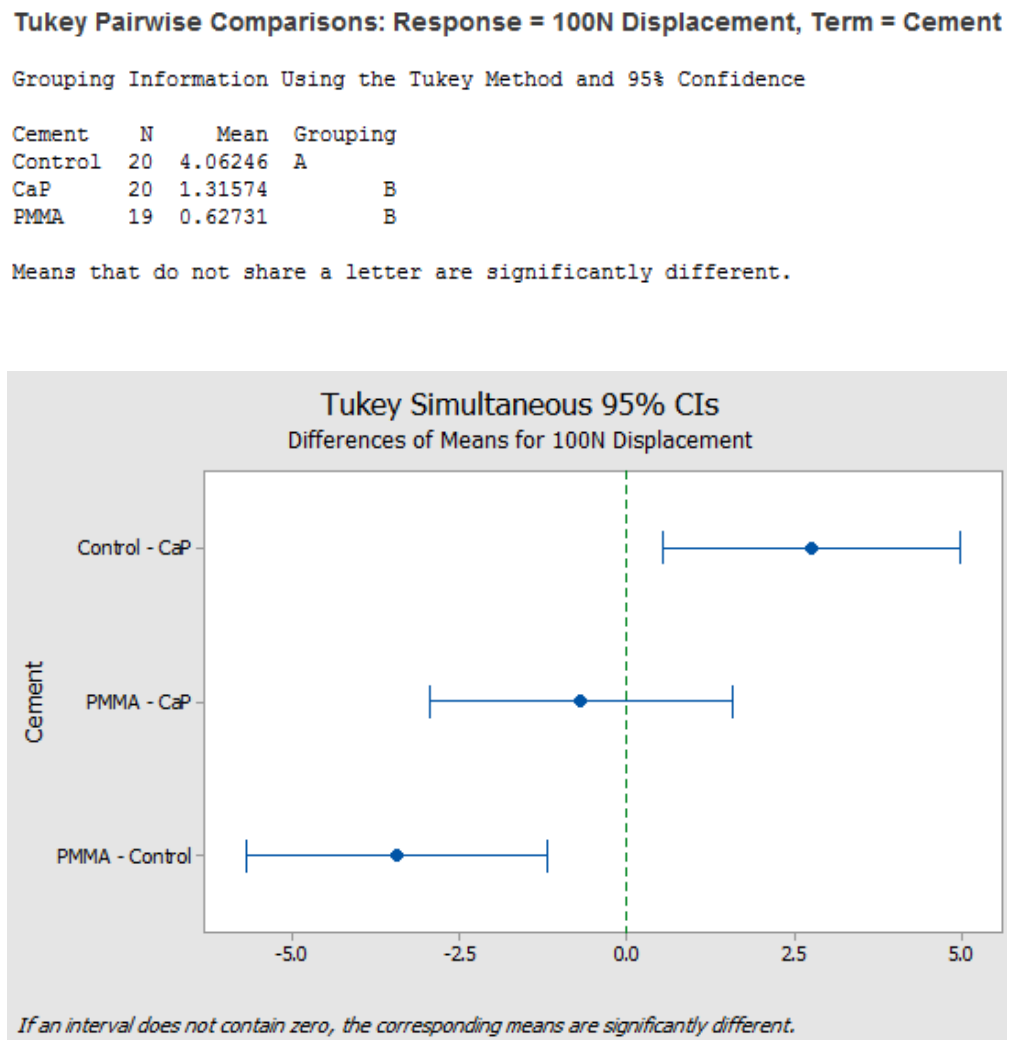

Figure 67: Tukey Pairwise comparison test of low density non-stripped screw samples for Adjusted Displacement at the $100 \mathrm{~N}$ load level. Top: grouping results show the control group displacement alone in group A, while calcium phosphate and PMMA cements were statistically grouped together in group B. Bottom: Differences of means intervals for the same data set. The PMMA - CaP interval passes through zero proving statistical indifference between the two cement types. A $95 \%$ confidence interval was used for these calculations and the mean values displayed in the top figure are fitted means due to outlier data points being removed from the data set.

At $100 \mathrm{~N}$, the adjusted displacements measured for all samples in the low density blocks for non-stripped screw holes were found to be statistically equal between PMMA and Calcium phosphate cement groups (at 95\% confidence). This comparison only encompasses a narrow window in the otherwise broad spectrum of factors involved in this study, but this window is the focal point of the experiment. Low density foam blocks closely resembled the bone density of osteoporotic human bone which is more likely to need cement augmentation around bone screws than healthy bone. $12 \mathrm{~mm}$ deficits or large holes (tight in control groups) which feature bone screws in space are preferred over a stripped hole for augmentation in clinical trials. 

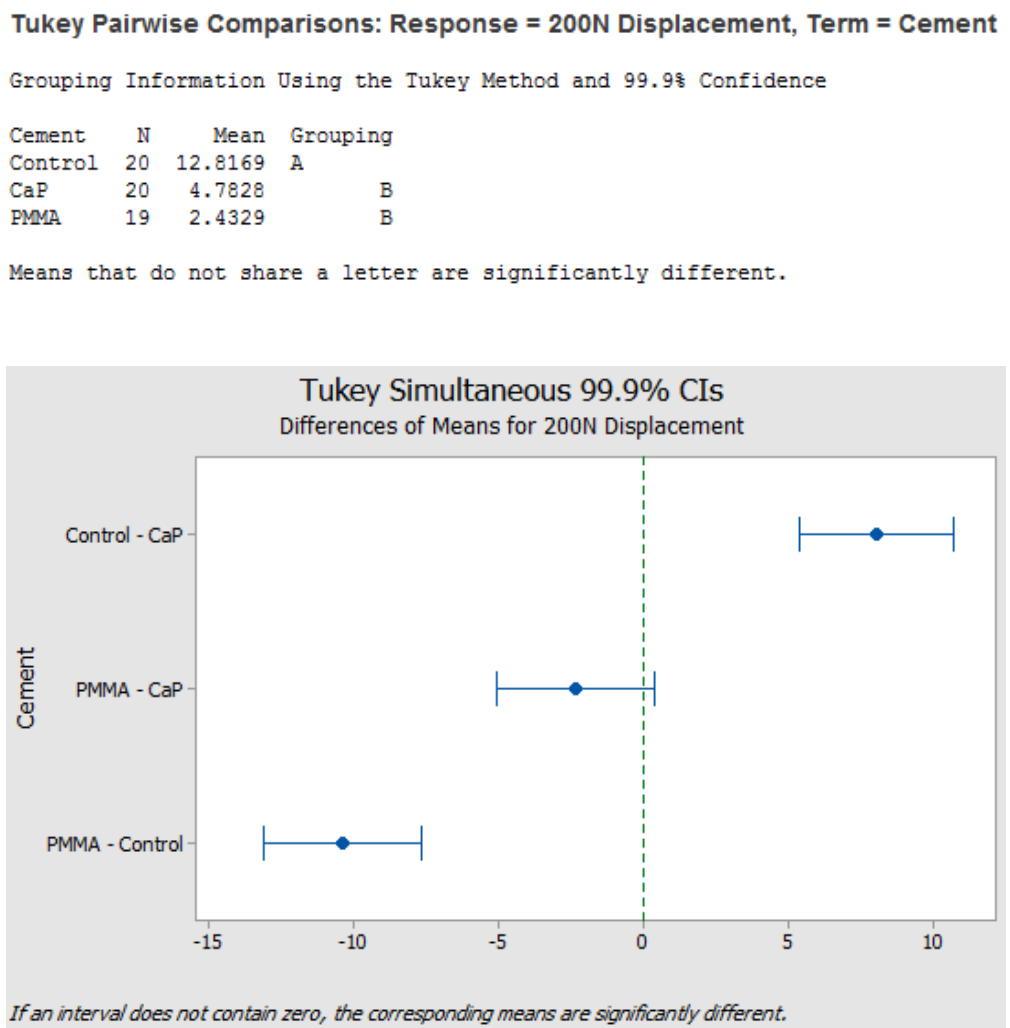

Figure 68: Tukey Pairwise comparison test of low density non-stripped screw samples for Adjusted Displacement at the 200N load level. Top: Fitted means and grouping for the three cement types. The control with no cement was grouped alone while both cements (PMMA and $\mathrm{CaP}$ ) were grouped together suggesting their means were statistically equal. Bottom: Differences of means intervals for the same data set. The PMMA - CaP interval passes through zero proving statistical indifference between the two cement types. A $99.9 \%$ confidence interval was used for these calculations and the mean values displayed in the top figure are fitted means due to outlier data points being removed from the data set.

Adjusted Displacement at the $200 \mathrm{~N}$ load level showed similar results to the $100 \mathrm{~N}$ displacements above. The fitted means were much different but the conclusion was the same: PMMA and CaP cements were statistically grouped together. The one difference in this comparison was the $99.9 \%$ confidence level. Both load levels of $100 \mathrm{~N}$ and $200 \mathrm{~N}$ were in the upper range of force a bone screw may be subject to in vivo while holding a locking plate in place. According to the 2 figures above (Figures 67 and 68) bone screws will exhibit the same amount of displacement on average while undergoing $100 \mathrm{~N}-200 \mathrm{~N}$ 
of cyclic loading regardless of whether they were augmented with PMMA cement or Calcium phosphate cement.

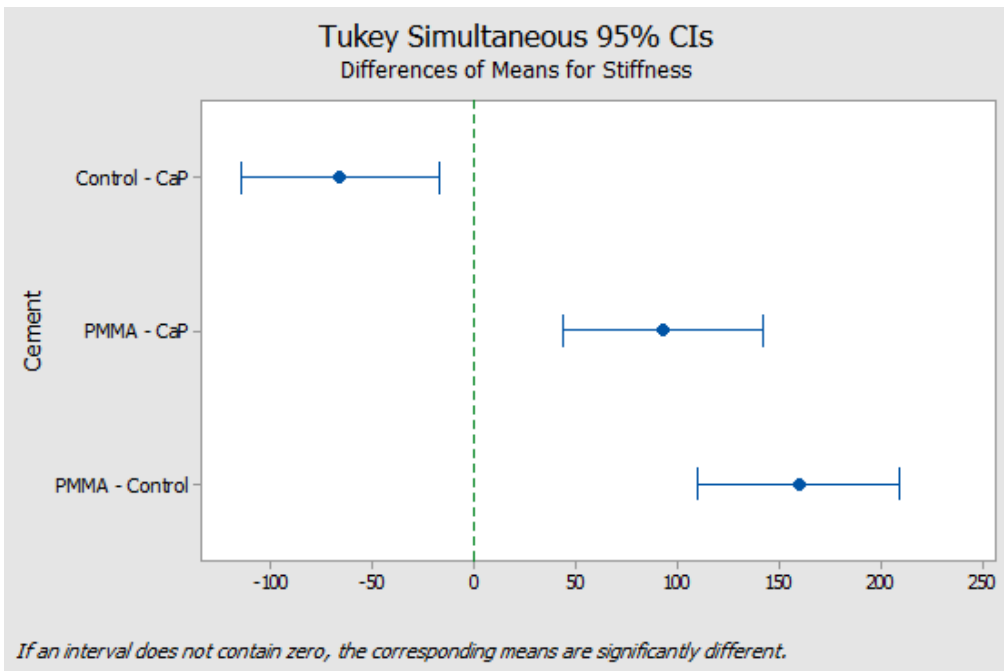

Figure 69: Fitted Means plot for Tukey Pairwise comparison of low density non-stripped screw samples for stiffness response at $95 \%$ confidence. None of the factor combinations pass through zero on this plot which means all three comparisons were in different statistical groups. The closest combination to zero was the Control - CaP comparison, followed by PMMA - CaP.

Unfortunately the stiffness response Tukey test comparison did not group any cement types together. The two most closely related types were the control group and CaP, followed by PMMA and CaP. At 99.9\% confidence the Tukey test groups Control and $\mathrm{CaP}$ together with PMMA separated. Increasing the confidence level also increased the critical value that the difference in means must be less than for two factor levels to be significantly different, so a higher confidence level forces factor means to be further apart to be confirmed as statistically different. The larger the confidence level the more likely that any two factors aren't deemed statistically different. 


\section{DISCUSSION}

\section{A. Unique Screw Pull-out Method}

The high density foam was a curious case in both large and stripped screw holes for calcium phosphate cement samples. The $30 \mathrm{lbs} / \mathrm{ft}^{3}$ blocks were distinctly smoother and denser than the low and medium (12.5 and $20 \mathrm{lbs} / \mathrm{ft}^{3}$ respectively) counterparts as one would expect. As it happens, calcium phosphate cement, once fully cured, was also quite smooth. When the slick $\mathrm{CaP}$ set inside the high density voids with very little nooks and crannies with which to interdigitate, it tended to slide directly out of the block, still intact with the screw.
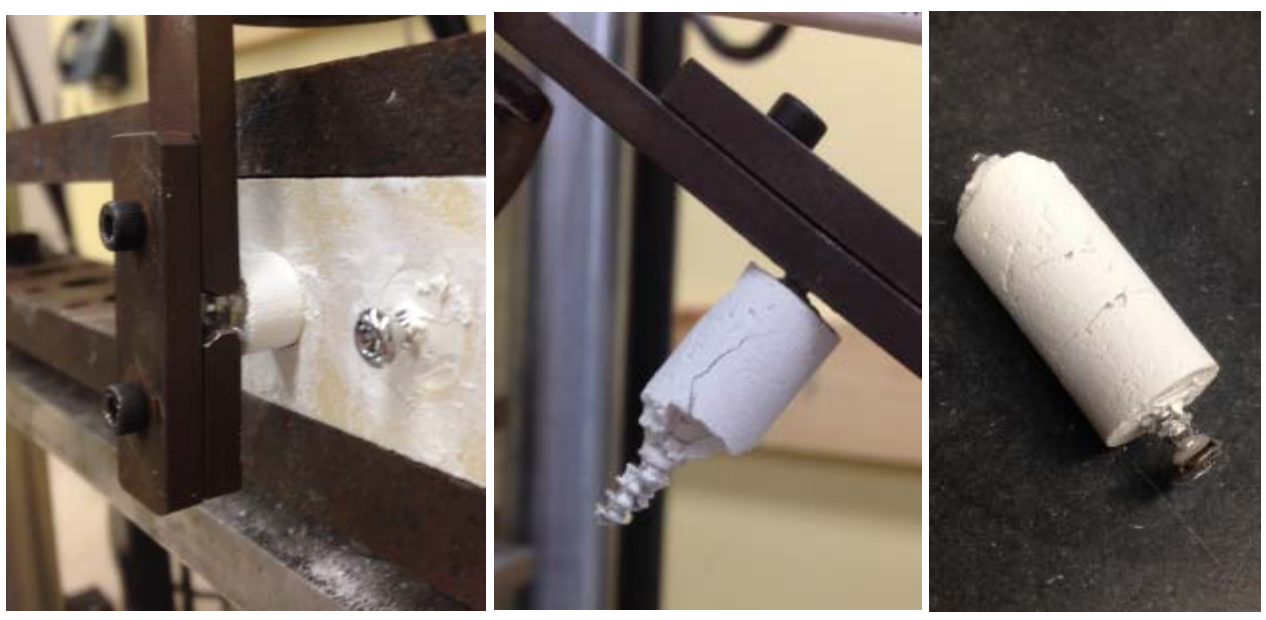

Figure 70: In the case of high density calcium phosphate $12 \mathrm{~mm}$ hole samples, the inherent nature of the $\mathrm{CaP}$ cement to cure to the shape of the container caused the cement samples to slide directly out of the smooth cylindrical voids of the high density foam blocks when subjected to direct pullout forces. The left and middle picture shown above depict a sample with the locking plate attached as this combination often provided the more intact $\mathrm{CaP}$ cylinders at the end, although the same phenomenon occurred with the nonlocking plate attached. 
Due to this phenomenon involving the calcium phosphate cement in the $30 \mathrm{lbs} / \mathrm{ft}^{3}$ foam block, the high density calcium phosphate samples in $12 \mathrm{~mm}$ holes were discontinued after 3 iterations for both locking and nonlocking trials. The predictable tendency of the entire screw cement interface to completely pull out of the foam block made additional samples unnecessary. An additional study will be needed to truly test the strength of this cement for higher than normal density bone. The cylindrical shape should be avoided for future studies to allow the calcium phosphate to bind correctly to the bone. This may be accomplished by either etching out crevices along the walls of the drilled out cylinder to provide "hand holds" to which the cement can secure, or abandon the cylindrical shape altogether and perhaps adopt a cone shape or something that will limit the perpendicular smooth walls that allows this type of cement pullout. This being said, it is appropriate to recognize that calcium phosphate cement is not recommended for use in healthy or above average density bone. Luckily, the chances of very healthy bone needing screw augmentation are little to none. However, if augmentation is needed for healthy bone, perhaps due to a birth defect causing a void or previous injury, PMMA cement is suggested over $\mathrm{CaP}$ cement until further studies are completed.

\section{B. Cancellous versus Cortical Screw Types}

As stated before in the Failure Mode under Locking Plate subsection of the Procedure section (page 23) a few liberties were taken when the screw types were chosen for this study. Instead of using standard smooth head cancellous bone screws for "non- 
locking" samples and threaded cortical locking screws for the "locking" samples, only the single smooth head type was chosen for use throughout the entire project. This method was carefully chosen in order to have a constant screw shape throughout all samples, but not without the knowledge that this violates a few standard rules regarding locking and non-locking screw types. This study was aimed at cancellous screws specifically, which are normally non-locking screws. Locking head screws are almost always cortical bone screws, used only in thick cortical bone. Studying a locked head screw in cancellous bone conditions appears irrelevant from a research standpoint. However, for this unique study, it was relevant. As stated before it was very important that the variables throughout this study were normalized so as to compare "apples to apples." Using two completely different screw types would add yet another degree of difference which would further blur the results across significant factor combinations.

\section{Literature Validation}

The results in the study by Amendola et al. [7] show that PMMA is, indeed, quite strong. So strong, in fact, that in 21 recorded cases of (pedicle) screw augmentation in patients with bone softening none of them exhibited signs of screw loosening or pullout. The screws used in that study had cannulated cores and were fenestrated with two sets of three holes in the distal portion of the thread. This no doubt provided the cement with extra gripping strength at the base of the screw. The comparison to be made here is with the large, $12 \mathrm{~mm}$ hole screw samples in PMMA cement and low quality foam blocks. 
None of the large hole samples in PMMA cement across all three densities pulled out during testing, although some of the screws broke. Comparing these results to an in vivo case study isn't entirely accurate, but it still demonstrates the strength of the PMMA cement across both studies.

In a study by Grawe B, Le T et al., [22] the strength of locking screws was compared to that of non-locking screws. In the study, two locking screws were compared to three nonlocking screws, with the results stating no significant differences between them. The takeaway from this was that a single locking screw is significantly stronger than a single nonlocking screw. This was also proven in the current study, as locking screws exhibited less displacement and more stiffness across all densities, hole sizes and cements $(\mathrm{P}=0.00)$. 


\section{CONCLUSION}

The conclusions to be drawn from this study are as follows:

1) The new toggle testing method is more clinically relevant because these results are more like the failure rates and failure modes seen clinically [7][10][12][17].

This new method has also revealed that screw fixation ability has been underestimated by direct pullout testing because it over taxes the screw-bone interface which is the weak link in poor quality bone.

2) This new test method confirms that locked screws perform better than non-locked screws in poor quality bone $(\mathrm{P}=0.00)$.

3) This study supports the use of calcium phosphate cement to augment screws in large defects and poor quality bone in addition the previously accepted technique of using PMMA. Pullout testing in the past has shown that calcium phosphate cement adequately helps secure bone screws [15] but this method has demonstrated that $\mathrm{CaP}$ can be just as reliable as PMMA in most cases.

4) Finally, stripped holes are much more difficult to reliably augment with cements than larger defects. 


\section{APPENDIX}

\section{Color Code Key:}

: Deemed outlier; removed from final results

: Error during sample, removed from final results

: Standard residual \pm 3.5 in $1^{\text {st }}$ round of modeling

: Standard residual \pm 3.0 in $1^{\text {st }}$ round of modeling

: Standard residual \pm 3.5 in $2^{\text {nd }}$ round of modeling

Breakage*: Screw broke just under head instead of down the threads 
Table XII: Control, no cement Low Density

\begin{tabular}{|c|c|c|c|c|c|c|}
\hline Trial & Cycles completed & Load at failure (N) & Avg displacement (mm) & Adjusted Displacement (mm) & Stiffness (N/mm) & Failure Mode \\
\hline Low Tight Locking 1 & 3642 & 200 & 6.548 & 6.025 & 33.192 & Breakage \\
\hline Low Tight Locking 2 & 3497 & 200 & 6.828 & 6.306 & 31.716 & Breakage \\
\hline Low Tight Locking 3 & 3381 & 200 & 6.410 & 5.887 & 33.973 & Breakage \\
\hline Low Tight Locking 4 & 3325 & 200 & 5.565 & 5.042 & 39.667 & Breakage \\
\hline Low Tight Locking 5 & 3457 & 200 & 6.577 & 6.054 & 33.036 & Breakage* \\
\hline Low Tight Locking 6 & 3311 & 200 & 15.892 & 15.370 & 13.013 & Pullout \\
\hline Low Tight Locking 7 & 3177 & 200 & 7.611 & 7.089 & 28.213 & Breakage \\
\hline Low Tight Locking 8 & 3278 & 200 & 8.666 & 8.143 & 24.560 & Breakage \\
\hline Low Tight Locking 9 & 3216 & 200 & 6.933 & 6.411 & 31.198 & Breakage \\
\hline Low Tight Locking 10 & 3318 & 200 & 7.271 & 6.749 & 29.636 & Breakage \\
\hline Low Tight Nonlocking 1 & 3253 & 200 & 19.926 & 19.403 & 10.308 & Pullout \\
\hline Low Tight Nonlocking 2 & 3212 & 200 & 17.759 & 17.237 & 11.603 & Pullout \\
\hline Low Tight Nonlocking 3 & 3195 & 200 & 18.994 & 18.471 & 10.828 & Pullout \\
\hline Low Tight Nonlocking 4 & 3123 & 200 & 18.103 & 17.580 & 11.377 & Pullout \\
\hline Low Tight Nonlocking 5 & 3209 & 200 & 18.672 & 18.149 & 11.020 & Pullout \\
\hline Low Tight Nonlocking 6 & 2502 & 100 & 16.557 & 16.332 & 6.123 & Pullout \\
\hline Low Tight Nonlocking 7 & 2484 & 100 & 16.335 & 16.110 & 6.207 & Pullout \\
\hline Low Tight Nonlocking 8 & 2679 & 100 & 15.624 & 15.400 & 6.494 & Pullout \\
\hline Low Tight Nonlocking 9 & 3188 & 200 & 17.787 & 17.265 & 11.584 & Pullout \\
\hline Low Tight Nonlocking 10 & 3234 & 200 & 20.703 & 20.181 & 9.911 & Pullout \\
\hline Low Stripped Locking 1 & 2392 & 100 & 3.495 & 3.270 & 30.584 & Pullout \\
\hline Low Stripped Locking 2 & 3001 & 200 & 3.438 & 2.915 & 68.609 & Pullout \\
\hline Low Stripped Locking 3 & 3008 & 200 & 4.559 & 4.037 & 49.545 & Pullout \\
\hline Low Stripped Locking 4 & 2007 & 100 & 2.932 & 2.707 & 36.944 & Pullout \\
\hline Low Stripped Locking 5 & 1001 & 50 & 0.681 & 0.579 & 86.317 & Pullout \\
\hline Low Stripped Locking 6 & 7 & 25 & 0.545 & 0.499 & 50.121 & Pullout \\
\hline Low Stripped Locking 7 & 1066 & 50 & 2.771 & 2.669 & 18.737 & Pullout \\
\hline Low Stripped Locking 8 & 3007 & 200 & 4.303 & 3.780 & 52.909 & Pullout \\
\hline Low Stripped Locking 9 & 1317 & 50 & 3.842 & 3.740 & 13.369 & Pullout \\
\hline Low Stripped Locking 10 & 3 & 25 & 0.000 & 0.000 & - & Pullout \\
\hline Low Stripped Nonlocking 1 & 1 & 25 & 0.462 & 0.416 & 60.150 & Pullout \\
\hline Low Stripped Nonlocking 2 & 1 & 25 & 0.686 & 0.640 & 39.090 & Pullout \\
\hline Low Stripped Nonlocking 3 & 1 & 25 & 0.957 & 0.911 & 27.440 & Pullout \\
\hline Low Stripped Nonlocking 4 & 1 & 25 & 0.734 & 0.688 & 36.337 & Pullout \\
\hline Low Stripped Nonlocking 5 & 1 & 25 & 0.647 & 0.601 & 41.595 & Pullout \\
\hline Low Stripped Nonlocking 6 & 1 & 25 & 1.265 & 1.219 & 20.510 & Pullout \\
\hline Low Stripped Nonlocking 7 & 1 & 25 & 0.774 & 0.728 & 34.317 & Pullout \\
\hline Low Stripped Nonlocking 8 & 4 & 25 & 1.000 & 0.954 & 26.201 & Pullout \\
\hline Low Stripped Nonlocking 9 & 1 & 25 & 0.761 & 0.715 & 34.949 & Pullout \\
\hline Low Stripped Nonlocking 10 & 1 & 25 & 1.058 & 1.012 & 24.713 & Pullout \\
\hline
\end{tabular}


Table XIII: Control, no cement Medium Density

$$
\text { Trial }
$$

Cycles Completed

Load at failure $(\mathrm{N})$

Avg displacement (mm)

Adjusted Displacement (mm)

Stiffness (N/mm)

Failure Mode

\section{Medium Tight Locking}

Medium Tight Locking 2

Medium Tight Locking 3

Medium Tight Locking 4

Medium Tight Locking 5

Medium Tight Locking 6

Medium Tight Locking 7

Medium Tight Locking 8

Medium Tight Locking 9

Medium Tight Locking 10

Medium Tight Nonlocking 1

Medium Tight Nonlocking 2

Medium Tight Nonlocking 3

Medium Tight Nonlocking 4

Medium Tight Nonlocking 5

Medium Tight Nonlocking 6

Medium Tight Nonlocking 7

Medium Tight Nonlocking 8

Medium Tight Nonlocking 9

Medium Tight Nonlocking 10

Medium Stripped Locking 1

Medium Stripped Locking 2

Medium Stripped Locking 3

Medium Stripped Locking 4

Medium Stripped Locking 5

Medium Stripped Locking 6

Medium Stripped Locking 7

Medium Stripped Locking 8

Medium Stripped Locking 9

Medium Stripped Locking 10

Medium Stripped Nonlocking 1

Medium Stripped Nonlocking 2

Medium Stripped Nonlocking 3

Medium Stripped Nonlocking 4

Medium Stripped Nonlocking 5

Medium Stripped Nonlocking 6

Medium Stripped Nonlocking 7

Medium Stripped Nonlocking 8

Medium Stripped Nonlocking 9

$\begin{array}{ll}4000 & 200 \\ 4000 & 200 \\ 3702 & 200 \\ 4000 & 200 \\ 4000 & 200 \\ 4000 & 200 \\ 4000 & 200 \\ 4000 & 200 \\ 3985 & 200 \\ 4000 & 200 \\ 3803 & 200 \\ 3955 & 200\end{array}$

$\begin{array}{ll}200 & 1.656 \\ 200 & 1.703 \\ 200 & 4.078\end{array}$

$\begin{array}{ll}1.656 & 1.134 \\ 1.703 & 1.180 \\ 4.078 & 3.555\end{array}$

1.134
1.180
3.555

$\begin{array}{ll}4.078 & 3.555 \\ 2.178 & 1.656\end{array}$

$\begin{array}{ll}200 & 2.178 \\ 200 & 2.398\end{array}$

$200 \quad 2.105$

2.101

2.388

4.084

1.582
1.578
1.865
3.562

$\begin{array}{ll}4.084 & 3.562 \\ 2.304 & 1.781\end{array}$

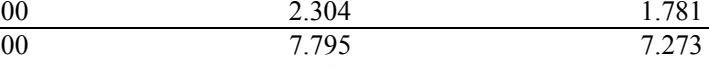

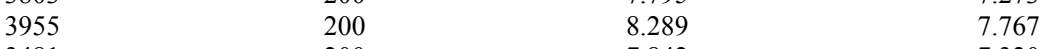

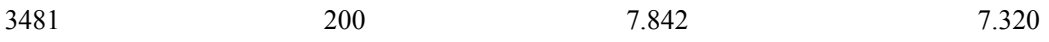

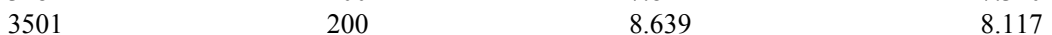

$\begin{array}{ll}3501 & 8.639 \\ 3983 & 200\end{array}$

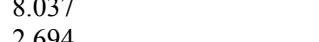

2.694

$4.742-4.220$

$\begin{array}{ll}8.136 & 7.613 \\ 7.800 & 7.277\end{array}$

\begin{tabular}{|c|c|}
\hline 3571 & 200 \\
\hline
\end{tabular}

$\begin{array}{llll}3455 & 200 & 8.013 & 7.491 \\ 3236 & 200 & 4.764 & 4.242\end{array}$

3004200

4.465

4.465
4.356

4.356
3.617

$\begin{array}{lll}3336 & 200 & 4.356 \\ 3392 & 200 & 3.617\end{array}$

$\begin{array}{lll}3392 & 200 & 3.617 \\ 3264 & 200 & 3.848\end{array}$

3.848

$\begin{array}{lll}3334 & 200 & 4.708 \\ 3295 & 200 & 3.514\end{array}$

3.581
$3.514-4.185$
4.073
2.991

$\begin{array}{cccc}3295 & 200 & 3.573 & 3.550 \\ 3697 & 200 & 3.772 & 3.249 \\ 3118 & 25 & 2.243 & 2.197\end{array}$

$\begin{array}{ll}25 & 2.243 \\ 25 & 2.229\end{array}$

$\begin{array}{ll}2.773 & 2.197 \\ 2.229 & 2.183\end{array}$

$\begin{array}{lr}2.183 \\ 2.608 & 2.562 \\ 1.702 & 1.656\end{array}$

$\begin{array}{ll}2.608 & 2.562 \\ 1.702 & 1.656\end{array}$

$\begin{array}{ll}1.702 & 1.656 \\ 1.341 & 1.295\end{array}$

$\begin{array}{ll}1.341 & 1.295 \\ 2.577 & 2531\end{array}$

$\begin{array}{ll}2.577 & 2.531 \\ 2.419 & 2373\end{array}$

$\begin{array}{ll}2.419 & 2.373 \\ 2.250 & 2.204\end{array}$

$\begin{array}{ll}2.250 & 2.204 \\ 3.318 & 3.272\end{array}$

Medium Stripped Nonlocking 10

\begin{tabular}{ll}
25 & 3.318 \\
25 & 1.795 \\
\hline
\end{tabular}

3.272
1.749

.491

942

176.411
169.460
56.257
120.801

Survived

Survived
Breakage

Survived
Survived

$\begin{array}{ll}106.624 & \text { Survived } \\ 126.403 & \text { Survived }\end{array}$

126.734 Survived

$107.215 \quad$ Survived

$56.153 \quad$ Breakage*

$\begin{array}{ll}56.153 & \text { Breakage* } \\ 112.296 & \text { Survived }\end{array}$

$112.296 \quad$ Survived

$\begin{array}{ll}27.499 & \text { Breakage } \\ 25.752 & \text { Breakage }\end{array}$

$27.323 \quad$ Breakage

$24.640 \quad$ Breakage

26.617 Breakage

$\begin{array}{ll}26.617 & \text { Breakage } \\ 92.125 & \text { Survived }\end{array}$

$\begin{array}{ll}92.125 & \text { Survived } \\ 47.397 & \text { Survived }\end{array}$

47.397 Survived

$\begin{array}{ll}26.270 & \text { Breakage } \\ 27.483 & \text { Breakage }\end{array}$

\begin{tabular}{ll}
27.483 & Breakage \\
26.700 & Breakage \\
\hline 47.151 & Pullout
\end{tabular}

$\begin{array}{ll}47.151 & \text { Pullout } \\ 50.736 & \text { Pullout }\end{array}$

$\begin{array}{ll}50.736 & \text { Pullout } \\ 52.172 & \text { Pullout }\end{array}$

64.637 Pullout

$\begin{array}{ll}64.637 & \text { Pullout } \\ 27.921 & \text { Pullout } \\ 47.787 & \text { Pullout } \\ 6 & \text { Pullout }\end{array}$

$\begin{array}{ll}47.787 & \text { Pullout } \\ 66.863 & \text { Pullout } \\ 56.333 & \text { Pullout }\end{array}$

\begin{tabular}{ll}
66.863 & Pullout \\
56.333 & Pullout \\
61.553 & Pullout \\
\hline 11.378 & Pullout
\end{tabular}

$\begin{array}{cc}61.553 & \text { Pullout } \\ 11.378 & \text { Pullout } \\ 11.450 & \text { Pullout } \\ 9.756 & \text { Pullout }\end{array}$

$\begin{array}{cc}11.450 & \text { Pullout } \\ 9.756 & \text { Pullout } \\ 15.093 & \text { Pullout } \\ 19.310 & \text { Pullout } \\ 9.876 & \text { Pullout }\end{array}$

$\begin{array}{ll}19.310 & \text { Pullout } \\ 9.876 & \text { Pullout }\end{array}$

$\begin{array}{cc}9.876 & \text { Pullout } \\ 10.535 & \text { Pullout } \\ 1.342 & \text { Pullout }\end{array}$

$\begin{array}{ll}10.535 & \text { Pullout } \\ 11.342 & \text { Pullout }\end{array}$

\begin{tabular}{cl}
7.640 & Pullout \\
14.290 & Pullout \\
\hline
\end{tabular}


Table XIV: Control, no cement High Density

\begin{tabular}{|c|c|c|c|c|c|c|}
\hline Trial & Cycles Completed & Load at failure (N) & Avg displacement (mm) & Adjusted Displacement (mm) & Stiffness (N/mm) & Failure Mode \\
\hline High Tight Locking 1 & 4000 & 200 & 0.832 & 0.309 & 647.236 & Survived \\
\hline High Tight Locking 2 & 4000 & 200 & 0.913 & 0.390 & 512.418 & Survived \\
\hline High Tight Locking 3 & 4000 & 200 & 1.031 & 0.508 & 393.607 & Survived \\
\hline High Tight Locking 4 & 4000 & 200 & 1.033 & 0.511 & 391.483 & Survived \\
\hline High Tight Locking 5 & 4000 & 200 & 1.123 & 0.601 & 332.895 & Survived \\
\hline High Tight Locking 6 & 4000 & 200 & 1.102 & 0.580 & 344.961 & Survived \\
\hline High Tight Locking 7 & 4000 & 200 & 1.109 & 0.586 & 341.311 & Survived \\
\hline High Tight Locking 8 & 4000 & 200 & 1.275 & 0.752 & 265.829 & Survived \\
\hline High Tight Locking 9 & 4000 & 200 & 1.359 & 0.836 & 239.114 & Survived \\
\hline High Tight Locking 10 & 4000 & 200 & 1.443 & 0.920 & 217.279 & Survived \\
\hline High Tight Nonlocking 1 & 4000 & 200 & 0.740 & 0.217 & 920.080 & Survived \\
\hline High Tight Nonlocking 2 & 3575 & 200 & 5.333 & 4.810 & 41.576 & Breakage \\
\hline High Tight Nonlocking 3 & 3742 & 200 & 5.313 & 4.791 & 41.747 & Breakage \\
\hline High Tight Nonlocking 4 & 4000 & 200 & 1.225 & 0.702 & 284.872 & Survived \\
\hline High Tight Nonlocking 5 & 4000 & 200 & 1.673 & 1.150 & 173.875 & Survived \\
\hline High Tight Nonlocking 6 & 3491 & 200 & 5.583 & 5.061 & 39.521 & Breakage \\
\hline High Tight Nonlocking 7 & 4000 & 200 & 2.065 & 1.543 & 129.649 & Survived \\
\hline High Tight Nonlocking 8 & 3267 & 200 & 5.552 & 5.030 & 39.765 & Breakage \\
\hline High Tight Nonlocking 9 & 3254 & 200 & 5.600 & 5.078 & 39.387 & Breakage \\
\hline High Tight Nonlocking 10 & 4000 & 200 & 2.603 & 2.081 & 96.121 & Survived \\
\hline High Stripped Locking 1 & 2074 & 100 & 3.594 & 3.370 & 29.677 & Pullout \\
\hline High Stripped Locking 2 & 1173 & 50 & 2.634 & 2.531 & 19.751 & Pullout \\
\hline High Stripped Locking 3 & 1669 & 50 & 3.344 & 3.242 & 15.422 & Pullout \\
\hline High Stripped Locking 4 & 1221 & 50 & 2.832 & 2.730 & 18.313 & Pullout \\
\hline High Stripped Locking 5 & 1023 & 50 & 2.963 & 2.861 & 17.478 & Pullout \\
\hline High Stripped Locking 6 & 1530 & 50 & 2.805 & 2.703 & 18.500 & Pullout \\
\hline High Stripped Locking 7 & 321 & 25 & 0.856 & 0.810 & 30.849 & Pullout \\
\hline High Stripped Locking 8 & 2007 & 100 & 3.403 & 3.178 & 31.462 & Pullout \\
\hline High Stripped Locking 9 & 1341 & 50 & 3.335 & 3.233 & 15.466 & Pullout \\
\hline High Stripped Locking 10 & 1068 & 50 & 4.460 & 4.358 & 11.473 & Pullout \\
\hline High Stripped Nonlocking 1 & 3 & 25 & 1.161 & 1.115 & 22.423 & Pullout \\
\hline High Stripped Nonlocking 2 & 3 & 25 & 2.856 & 2.810 & 8.895 & Pullout \\
\hline High Stripped Nonlocking 3 & 4 & 25 & 1.743 & 1.697 & 14.731 & Pullout \\
\hline High Stripped Nonlocking 4 & 3 & 25 & 1.647 & 1.601 & 15.618 & Pullout \\
\hline High Stripped Nonlocking 5 & 2 & 25 & 2.223 & 2.177 & 11.486 & Pullout \\
\hline High Stripped Nonlocking 6 & 2 & 25 & 2.512 & 2.466 & 10.138 & Pullout \\
\hline High Stripped Nonlocking 7 & 2 & 25 & 2.653 & 2.607 & 9.589 & Pullout \\
\hline High Stripped Nonlocking 8 & 2 & 25 & 2.657 & 2.611 & 9.576 & Pullout \\
\hline High Stripped Nonlocking 9 & 3 & 25 & 2.333 & 2.287 & 10.932 & Pullout \\
\hline High Stripped Nonlocking 10 & 2 & 25 & 2.347 & 2.301 & 10.867 & Pullout \\
\hline
\end{tabular}


Table XV: PMMA Cement Low Density

Trial

\begin{tabular}{l}
\hline Large Low Locking 1 \\
Large Low Locking 2
\end{tabular}

Large Low Locking 3

Large Low Locking 4

Large Low Locking 5

Large Low Locking 6

Large Low Locking 7

Large Low Locking 8

Large Low Locking 9

Large Low Locking 10

Large Low Nonlocking 1

Large Low Nonlocking 2

Large Low Nonlocking 3

Large Low Nonlocking 4

Large Low Nonlocking 5

Large Low Nonlocking 6

Large Low Nonlocking 7

Large Low Nonlocking 8

Large Low Nonlocking 9

Large Low Nonlocking 10

Stripped Low Locking 1

Stripped Low Locking 2

Stripped Low Locking 3

Stripped Low Locking 4

Stripped Low Locking 5

Stripped Low Locking 6

Stripped Low Locking 7

Stripped Low Locking 8

Stripped Low Locking 9

Stripped Low Locking 10

Stripped Low Nonlocking 1

Stripped Low Nonlocking 2

Stripped Low Nonlocking 3

Stripped Low Nonlocking 4

Stripped Low Nonlocking 5

Stripped Low Nonlocking 6

Stripped Low Nonlocking 7

Stripped Low Nonlocking 8

Stripped Low Nonlocking 9

Stripped Low Nonlocking 10

Cycles until failure Load at failure (N)

Avg displacement (mm)

Adjusted Displacement (mm)

Stiffness (N/mm)

416.477
356.834

0.480
0.560

$\begin{array}{lll}4000 & 200 & 1.003 \\ 4000 & 200 & 1.083\end{array}$

$\begin{array}{lll}4000 & 200 & 1.083\end{array}$

0.562

355.741

1.253

$4000 \quad 200$

$200 \quad 1.775$

1.775
1.019

$\begin{array}{lrl}4000 & 200 & 1.019 \\ 4000 & 200 & 1.274\end{array}$

$\begin{array}{lll}4000 & 200 & 1.274 \\ 4000 & 200 & 1.331\end{array}$

\begin{tabular}{lll}
4000 & 200 & 1.331 \\
4000 & 200 & 1.404 \\
\hline
\end{tabular}

$\begin{array}{ll}4000 & 200 \\ 3540 & 200\end{array}$

$\begin{array}{ll}200 & 1.404 \\ 200 & 1.218\end{array}$

$\begin{array}{lll}35000 & 200 & 5 . \\ 4000 & 200 & 1.885\end{array}$

$4000 \quad 200$

$3410 \quad 200$

$3816 \quad 200$

$\begin{array}{ll}3802 & 200 \\ 3790 & 200 \\ 3513 & 200\end{array}$

3790

3513

$3065-200$

$3036 \quad 200$

$4000 \quad 200$

$4000 \quad 200 \quad 1$

$\begin{array}{lll}4000 & 200 & 2 . \\ 4000 & 200 & 2.007\end{array}$

$\begin{array}{ll}3774 & 200 \\ 4000 & 200\end{array}$

$4000 \quad 200$

$\begin{array}{rr}4000 \\ 4000 & 200 \\ 4000 & 200\end{array}$

$\begin{array}{ll}4000 & 200 \\ 4000 & 200\end{array}$

$\begin{array}{ll}4000 & 200 \\ 3234 & 200\end{array}$

3102

3438

3016

3016

3390

3023
3267

3381

3656

$200 \quad 1.862$

1.218

$5.150 \quad 4.627$

1.885

1.853

5.289
5.383

5.383
5.137

$5.137 \quad 4.614$

$5.082 \quad 4.560$

$4.680 \quad 4.157$

$\begin{array}{ll}6.534 & 6.012 \\ 7.481 & 6.958\end{array}$

$\begin{array}{ll}6.534 & 6.958 \\ 1.964 & 1.441\end{array}$

$\begin{array}{ll}1.655 & 1.132\end{array}$

$\begin{array}{ll}1.413 & 1.890 \\ 2.007 & 1.485\end{array}$

$2.007-1.48$

$\begin{array}{ll}9.129 & 8.606 \\ 1.757 & 1.235 \\ 1.832 & 1.309\end{array}$

$\begin{array}{ll}1.757 & 1.235 \\ 1.832 & 1.309 \\ 1.800 & 1.277\end{array}$

$\begin{array}{ll}1.800 & 1.277 \\ 1.531 & 1.009\end{array}$

$\begin{array}{ll}1.531 & 1.009 \\ 1.862 & 1.340\end{array}$

$\begin{array}{cc}1.862 & 1.340 \\ 11.964 & 11.442 \\ 11.871 & 11.348 \\ 13.412 & 13.188\end{array}$

$\begin{array}{cc}11.871 & 11.348 \\ 13.412 & 13.188 \\ 7.578 & 7.055 \\ 9.756 & 9.234\end{array}$

$\begin{array}{rr}7.578 & 7.055 \\ 9.756 & 9.234\end{array}$

$\begin{array}{lr}9.756 & 9.234 \\ 7.582 & 7.060\end{array}$

$\begin{array}{cc}7.582 & 7.060 \\ 11.219 & 10.696 \\ 6.594 & 6.071\end{array}$

\begin{tabular}{ll}
6.594 & 6.071 \\
6.856 & 6.333 \\
5.774 & 5.252 \\
\hline
\end{tabular}

6.333
5.252

159.673

$\begin{array}{ll}159.673 & \text { Survived } \\ 402.614 & \text { Survived } \\ 266.316 & \text { Survived }\end{array}$

$266.316 \quad$ Survived

247.472 Survived

$226.874 \quad$ Survived

$\begin{array}{ll}226.874 & \text { Survived } \\ 287.553 & \text { Survived }\end{array}$

\begin{tabular}{ll}
287.553 & Survived \\
\hline 43.223 & Breakage
\end{tabular}

$\begin{array}{ll}146.794 & \text { Survived }\end{array}$

$150.290 \quad$ Survived

41.961 Breakage

41.146 Breakage

41.146 Breakage
43.342

43.342 Breakage

$\begin{array}{ll}43.863 & \text { Breakage } \\ 48.108 & \text { Breakage } \\ 33.269 & \text { Breakage }\end{array}$

$\begin{array}{ll}33.269 & \text { Breakage } \\ 28.744 & \text { Beakage }\end{array}$

$28.744 \quad$ Breakage

$\begin{array}{ll}138.793 & \text { Survived } \\ 176.680 & \text { Survived }\end{array}$

$\begin{array}{ll}176.680 & \text { Survived } \\ 105.808 & \text { Survived }\end{array}$

$\begin{array}{cc}105.808 & \text { Survived } \\ 134.703 & \text { Survived } \\ 23.240 & \text { Pullout } \\ 161.989 & \text { Survived }\end{array}$

$\begin{array}{cc}23.240 & \text { Pullout } \\ 161.989 & \text { Survived }\end{array}$

$\begin{array}{ll}161.989 & \text { Survived } \\ 152.741 & \text { Survived } \\ 156.614 & \text { Survived } \\ 198.282 & \text { Survived }\end{array}$

\begin{tabular}{cc}
156.614 & Survived \\
198.282 & Survived \\
149.285 & Survived \\
\hline 17.480 & Pullout
\end{tabular}

$\begin{array}{cc}149.285 & \text { Survived } \\ 17.480 & \text { Pullout } \\ 17.624 & \text { Pullout } \\ 7.583 & \text { Pullout }\end{array}$

$\begin{array}{ll}17.624 & \text { Pullout } \\ 7.583 & \text { Pullout } \\ 28.348 & \text { Breklog }\end{array}$

$\begin{array}{cc}7.583 & \text { Pullout } \\ 28.348 & \text { Breakage } \\ 21.660 & \text { Pullout } \\ 28.330 & \text { Breakage }\end{array}$

$\begin{array}{lc}21.660 & \text { Pullout } \\ 28.330 & \text { Breakage }\end{array}$

$\begin{array}{ll}18.698 & \text { Breakage } \\ 32.942 & \text { Pullout } \\ 31.580 & \text { Breakage }\end{array}$

$\begin{array}{lc}32.942 & \text { Pullout } \\ 31.580 & \text { Breakage } \\ 38.083 & \text { Breakage }\end{array}$

\begin{tabular}{ll}
31.580 & Breakage \\
38.083 & Breakage \\
\hline
\end{tabular}


Table XVI: PMMA Cement Medium Density

Trial

Stripped Medium Locking 1

Stripped Medium Locking 2

Stripped Medium Locking 3

Stripped Medium Locking 4

Stripped Medium Locking 5

Stripped Medium Locking 6

Stripped Medium Locking 7

Stripped Medium Locking 8

Stripped Medium Locking 9

Stripped Medium Locking 10

Stripped Medium Nonlocking 1

Stripped Medium Nonlocking 2

Stripped Medium Nonlocking 3

Stripped Medium Nonlocking 4

Stripped Medium Nonlocking 5

Stripped Medium Nonlocking 6

Stripped Medium Nonlocking 7

Stripped Medium Nonlocking 8

Stripped Medium Nonlocking 9

Stripped Medium Nonlocking 10

Large Medium Locking 1

Large Medium Locking

Large Mediun Locking

Large Medium Locking 4

Large Medium Locking 5

Large Medium Locking 6

Large Medium Locking 7

Large Medium Locking 8

Large Medium Locking 9

Large Medium Locking 10

Large Medium Nonlocking 1

Large Medium Nonlocking 2

Large Medium Nonlocking 3

Large Medium Nonlocking 4

Large Medium Nonlocking 5

Large Medium Nonlocking 6

Large Medium Nonlocking 7

Large Medium Nonlocking 8

Large Medium Nonlocking 9

Large Medium Nonlocking 10

Cycles Completed Load at failure (N)

Avg displacement (mm)

Adjusted Displacement (mm)

Stiffness (N/mm) 287.268

287.268

250.353
294.108

294.108
239.707

305.081

1.357

1.178

4000

4000

200

200
200

$\begin{array}{rr}1485 & 50 \\ 4000 & 200\end{array}$

1.208

$\begin{array}{ll}500 & 2.809 \\ 200 & 0.992\end{array}$

4000

4000

2355

200

$200 \quad 1.447$

0.686
2.706
0.469

$\begin{array}{ll}1.745 & 0.469 \\ 1.447 & 1.223\end{array}$

$\begin{array}{ll}1.447 & 0.924 \\ 2.758 & 2.533\end{array}$

$\begin{array}{ll}100 & 2.758 \\ 50 & 2.603 \\ 200 & 1.555\end{array}$

$\begin{array}{lll}276 & 25 & 2.603 \\ 1348 & 50 & 1.555\end{array}$

$3023-200 \quad 7.097$

$\begin{array}{lr}3023 & 200 \\ 355 & 25\end{array}$

$3438 \quad 200$

2689

25

7.097
0.947
12.107

1.575
0.901

$12.107 \quad 11.585$

4.134
1.909

$\begin{array}{ll}1.767 & 1.665 \\ 7.392 & 6.869\end{array}$

\begin{tabular}{llll}
1244 & 500 & 7.392 & 6.869 \\
4000 & 200 & 3.908 & 3.385 \\
\hline
\end{tabular}

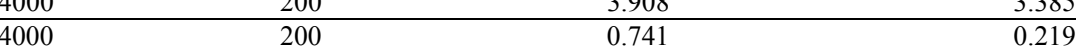

$\begin{array}{lll}4000 & 200 & 0.74 \\ 4000 & 200 & 0.762\end{array}$

$0.762-0.219$

$\begin{array}{llll}4000 & 200 & 0.762 & 0.240 \\ 4000 & 200 & 0.848 & 0.326\end{array}$

4000

4000

4000
4000

4000

4000

4000

3500

4000

3943

3765

3547

4000

4000

3915

3825

4000

0.848

0.837

0.744
0.988

0.888

0.832

0.967

0.994

4.356
1.486

1.486
4.701

4.292

4.333

1.351

1.640

4.595

4.582

1.811

0.240
0.326
0.315

0.315
0.222

0.222
0.465

$\begin{array}{lll}0.315 & 901.504 & \text { Survived } \\ 0.465 & 429.733 & \text { Survived }\end{array}$

$\begin{array}{lll}0.366 & 547.190 & \text { Survived }\end{array}$

$\begin{array}{lll}0.310 & 645.796 & \text { Survived } \\ 0.444 & 450.054 & \text { Survived }\end{array}$

$\begin{array}{lll}0.472 & 450.054 & \text { Survived } \\ 0.424 .083 & \text { Survived }\end{array}$

$\begin{array}{ccc}3.834 & 52.167 & \text { Breakage } \\ 0.963 & 207.643 & \text { Survived }\end{array}$

$\begin{array}{ccc}0.963 & 207.643 & \text { Survived } \\ 4.178 & 47.866 & \text { Breakage }\end{array}$

$\begin{array}{lll}4.178 & 47.866 & \text { Breakage } \\ 3.769 & 53.059 & \text { Breakage } \\ 3.811 & 52.483 & \text { Breakage }\end{array}$

$\begin{array}{lll}3.769 & 53.059 & \text { Breakage } \\ 3.811 & 52.483 & \text { Breakage }\end{array}$

$\begin{array}{lrr}3.811 & 52.483 & \text { Breakage } \\ 0.828 & 241.501 & \text { Survived }\end{array}$

$\begin{array}{lll}0.828 & 241.501 & \text { Survived } \\ 1.118 & 178.967 & \text { Survived }\end{array}$

$\begin{array}{lll}1.118 & 178.967 & \text { Survived } \\ 4.073 & 49.109 & \text { Breakege }\end{array}$

$\begin{array}{lll}4.073 & 49.109 & \text { Breakage } \\ 4.059 & 49.268 & \text { Breakage }\end{array}$

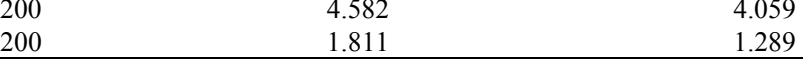

155.191

Breakage
Survived 
Table XVII: PMMA Cement High Density

\begin{tabular}{|l} 
Trial \\
\hline Stripped High Locking 1
\end{tabular}

Stripped High Locking 2

Stripped High Locking 3

Stripped High Locking 4

Stripped High Locking 5

Stripped High Locking 6

Stripped High Locking 7

Stripped High Locking 8

Stripped High Locking 9

Stripped High Locking 10

Stripped High Nonlocking 1

Stripped High Nonlocking 2

Stripped High Nonlocking 3

Stripped High Nonlocking 4

Stripped High Nonlocking 5

Stripped High Nonlocking 6

Stripped High Nonlocking 7

Stripped High Nonlocking 8

Stripped High Nonlocking 9

Stripped High Nonlocking 10

Large High Locking 1

Large High Locking 2

Large High Locking 3

Large High Locking 4

Large High Locking 5

Large High Locking 6

Large High Locking 7

Large High Locking 8

Large High Locking 9

Large High Locking 10

Large High Nonlocking 1

Large High Nonlocking 2

Large High Nonlocking 3

Large High Nonlocking 4

Large High Nonlocking 5

Large High Nonlocking 6

Large High Nonlocking 7

Large High Nonlocking 8
Large High Nonlocking 9

Large High Nonlocking 10

Cycles Completed

Load at failure $(\mathrm{N})$

Avg displacement (mm)

Adjusted Displacement (mm)

Stiffness (N/mm)

205.657
194.561
277.121

277.121

372.160

292.478

318.819

233.254

265.585

$\begin{array}{lll}0.652 & 306.693 & \text { Survived } \\ 0.781 & 255.983 & \text { Survived }\end{array}$

$\begin{array}{ll}4000 & 200 \\ 4000 & 200\end{array}$

$\begin{array}{ll}4000 & 200 \\ 4000 & 200\end{array}$

\begin{tabular}{ll}
4000 & 200 \\
4000 & 200 \\
\hline
\end{tabular}

$3358 \quad 200$

3567

4000

3408

3408

3280

3193

3288

4000

4000

$3295-200$

200

$4000-200$

$4000 \quad 200$

$4000 \quad 200$

$4000 \quad 200$

$4000 \quad 200$

$\begin{array}{ll}4000 & 200\end{array}$

$\begin{array}{ll}4000 & 200 \\ 4000 & 200\end{array}$

$4000+200$

$4000-200$

$\begin{array}{rr}4000 & 200 \\ 4000 & 200\end{array}$

$\begin{array}{ll}4000 & 200 \\ 4000 & 200\end{array}$

$\begin{array}{ll}4000 & 200 \\ 3759 & 200\end{array}$

$\begin{array}{ll}3759 & 200\end{array}$

$\begin{array}{ll}3000 & 200 \\ 3999\end{array}$

$\begin{array}{ll}4000 & 200 \\ 3999 & 200\end{array}$

$\begin{array}{ll}3999 & 200 \\ 3371 & 200\end{array}$

$3371 \quad 200$

\begin{tabular}{|c|c|c|c|c|c|}
\hline 4000 & 200 & 1.435 & 0.913 & 219.165 & Survived \\
\hline 3533 & 200 & 4.541 & 4.018 & 49.770 & Breakage \\
\hline 4000 & 200 & 1.533 & 1.011 & 197.876 & Survived \\
\hline 3471 & 200 & 4.405 & 3.882 & 51.515 & Breakage \\
\hline
\end{tabular}

\begin{tabular}{llllcc}
4000 & 200 & 1.435 & 0.913 & 219.165 & Survived \\
3533 & 200 & 4.541 & 4.018 & 49.770 & Breakage \\
4000 & 200 & 1.533 & 1.011 & 197.876 & Survived \\
3471 & 200 & 4.405 & 3.882 & 51.515 & Breakage \\
\hline
\end{tabular}

\begin{tabular}{|c|c|c|c|c|c|}
\hline 4000 & 200 & 1.435 & 0.913 & 219.165 & Survived \\
\hline 3533 & 200 & 4.541 & 4.018 & 49.770 & Breakage \\
\hline 4000 & 200 & 1.533 & 1.011 & 197.876 & Survived \\
\hline 3471 & 200 & 4.405 & 3.882 & 51.515 & Breakage \\
\hline
\end{tabular}

$\begin{array}{ll}4000 & 200 \\ 3471 & 200\end{array}$

\begin{tabular}{|c|c|c|c|c|c|}
\hline 4000 & 200 & 1.435 & 0.913 & 219.165 & Survived \\
\hline 3533 & 200 & 4.541 & 4.018 & 49.770 & Breakage \\
\hline 000 & 200 & 1.533 & 1.011 & 197.876 & Survived \\
\hline 3471 & 200 & 4.405 & 3.882 & 51.515 & Breakage \\
\hline
\end{tabular}

1.533
4.405

1.276
1.175
1.304

1.276
1.175
1.304

$\begin{array}{ll}1.304 & 0.78 \\ 5.792 & 5.26\end{array}$

$\begin{array}{lcc}0.781 & 255.983 & \text { Survived } \\ 5.269 & 37.956 & \text { Breakage }\end{array}$

$\begin{array}{lll}5.269 & 37.956 & \text { Breakage } \\ 4.446 & 44.988 & \text { Breakage }\end{array}$

$\begin{array}{ccc}4.446 & 44.988 & \text { Breakage } \\ 1.587 & 125.992 & \text { Survived }\end{array}$

$\begin{array}{lll}5.861 & 34.125 & \text { Burvived } \\ 5.890 & 33.957 & \text { Breake }\end{array}$

$\begin{array}{lll}5.890 & 33.957 & \text { Breakage }\end{array}$

$\begin{array}{lll}5.993 & 33.957 & \text { Breakage }\end{array}$

$\begin{array}{lcl}5.993 & 33.374 & \text { Breakage } \\ 6.192 & 32.301 & \text { Breakage } \\ 1.986 & 100.706 & \text { Survived }\end{array}$

$\begin{array}{ccc}1.986 & 100.706 & \text { Survived } \\ 2.053 & 97.427 & \text { Survived }\end{array}$

$\begin{array}{lll}2.053 & 97.427 & \text { Survived } \\ 6.635 & 30.142 & \text { Breakage }\end{array}$

$\begin{array}{ccc}6.635 & 30.142 & \text { Breakage } \\ 0.400 & 499.630 & \text { Survived }\end{array}$

$\begin{array}{lll}0.400 & 39.630 & \text { Survived } \\ 0.330 & 606.655 & \text { Survived }\end{array}$

$\begin{array}{lll}0.330 & 606.655 & \text { Survived } \\ 0.312 & 640.805 & \text { Survived }\end{array}$

$\begin{array}{lll}0.312 & 640.805 & \text { Survived } \\ 0.364 & 549.782 & \text { Survived }\end{array}$

$\begin{array}{lll}0.364 & 549.782 & \text { Survived }\end{array}$

$\begin{array}{lll}0.364 & 549.782 & \text { Survived } \\ 0.483 & 413.806 & \text { Survived } \\ 0.771 & 259.530 & \text { Survived }\end{array}$

$\begin{array}{lll}0.771 & 259.530 & \text { Survived } \\ 0.249 & 803.002 & \text { Survived }\end{array}$

$\begin{array}{lll}0.249 & 803.002 & \text { Survived } \\ 0.266 & 751.059 & \text { Survived } \\ 0.352 & 567.517 & \text { Survived }\end{array}$

\begin{tabular}{lll}
0.352 & 567.517 & Survived \\
0.409 & 488.695 & Survived \\
\hline 1.052 & 190.039 & Survived
\end{tabular}

$\begin{array}{lll}1.052 & 190.039 & \text { Survived } \\ 1.008 & 198.418 & \text { Survived } \\ 4.499 & 44.457 & \text { Breakage } \\ 1.088 & 183.841 & \text { Survived }\end{array}$

$\begin{array}{lll}4.499 & 44.457 & \text { Breakage } \\ 1.088 & 183.841 & \text { Survived }\end{array}$

$\begin{array}{lcc}1.088 & 183.841 & \text { Survived } \\ 4.187 & 47.772 & \text { Breakage } \\ 4.497 & 44.471 & \text { Breakage }\end{array}$

$\begin{array}{lcc}4.497 & 44.471 & \text { Breakage } \\ 0.913 & 219.165 & \text { Survived } \\ 4.018 & 49.770 & \text { Breakage }\end{array}$

\begin{tabular}{|c|c|c|c|c|c|}
\hline 4000 & 200 & 1.435 & 0.913 & 219.165 & Survived \\
\hline 3533 & 200 & 4.541 & 4.018 & 49.770 & Breakage \\
\hline 000 & 200 & 1.533 & 1.011 & 197.876 & Survived \\
\hline 3471 & 200 & 4.405 & 3.882 & 51.515 & Breakage \\
\hline
\end{tabular}


Table XVIII: CaP Cement Low Density

\begin{tabular}{|c|c|c|c|c|c|c|}
\hline Trial & Cycles Completed & Load at failure (N) & Avg displacement (mm) & Adjusted Displacement (mm) & Stiffness (N/mm) & Failure Mode \\
\hline RETEST Large Low Locking 1 & 4000 & 200 & 2.236 & 1.713 & 116.744 & Survived \\
\hline RETEST Large Low Locking 2 & 4000 & 200 & 1.616 & 1.094 & 182.857 & Survived \\
\hline RETEST Large Low Locking 3 & 4000 & 200 & 1.948 & 1.425 & 140.336 & Survived \\
\hline RETEST Large Low Locking 4 & 3354 & 200 & 4.292 & 3.769 & 53.064 & Pullout \\
\hline RETEST Large Low Locking 5 & 4000 & 200 & 1.504 & 0.981 & 203.780 & Survived \\
\hline RETEST Large Low Locking 6 & 4000 & 200 & 1.844 & 1.322 & 151.308 & Survived \\
\hline TEST Large Low Locking 7 & 4000 & 200 & 1.933 & 1.411 & 141.775 & Survived \\
\hline TEST Large Low Locking 8 & 3751 & 200 & 3.286 & 2.763 & 72.381 & Pullout \\
\hline TEST Large Low Locking 9 & 4000 & 200 & 1.675 & 1.152 & 173.563 & Survived \\
\hline TEST Large Low Locking 10 & 4000 & 200 & 1.394 & 0.872 & 229.383 & Survived \\
\hline RETEST Large Low Nonlocking 1 & 3167 & 200 & 7.504 & 6.981 & 28.649 & Breakage \\
\hline RETEST Large Low Nonlocking 2 & 3176 & 200 & 7.088 & 6.565 & 30.465 & Breakage \\
\hline RETEST Large Low Nonlocking 3 & 3317 & 200 & 7.479 & 6.957 & 28.750 & Breakage \\
\hline RETEST Large Low Nonlocking 4 & 3136 & 200 & 7.809 & 7.286 & 27.449 & Breakage \\
\hline RETEST Large Low Nonlocking 5 & 3059 & 200 & 9.268 & 8.745 & 22.870 & Pullout \\
\hline RETEST Large Low Nonlocking 6 & 3075 & 200 & 8.208 & 7.685 & 26.024 & Pullout \\
\hline TEST Large Low Nonlocking 7 & 3252 & 200 & 7.661 & 7.138 & 28.018 & Breakage \\
\hline TEST Large Low Nonlocking 8 & 3256 & 200 & 10.197 & 9.675 & 20.673 & Breakage \\
\hline TEST Large Low Nonlocking 9 & 3075 & 200 & 8.711 & 8.189 & 24.423 & Pullout \\
\hline TEST Large Low Nonlocking 10 & 3316 & 200 & 10.455 & 9.932 & 20.136 & Breakage \\
\hline Stripped Low Locking 1 & 3850 & 200 & 4.623 & 4.100 & 48.779 & Pullout \\
\hline Stripped Low Locking 2 & 3102 & 200 & 3.631 & 3.108 & 64.350 & Pullout \\
\hline Stripped Low Locking 3 & 3212 & 200 & 3.550 & 3.028 & 66.056 & Pullout \\
\hline Stripped Low Locking 4 & 2335 & 100 & 2.017 & 1.792 & 55.797 & Pullout \\
\hline Stripped Low Locking 5 & 3342 & 200 & 4.040 & 3.518 & 56.857 & Pullout \\
\hline Stripped Low Locking 6 & 3143 & 200 & 3.815 & 3.292 & 60.748 & Pullout \\
\hline Stripped Low Locking 7 & 3054 & 200 & 3.429 & 2.906 & 68.812 & Pullout \\
\hline Stripped Low Locking 8 & 2201 & 100 & 2.362 & 2.137 & 46.794 & Pullout \\
\hline Stripped Low Locking 9 & 3755 & 200 & 5.570 & 5.047 & 39.624 & Breakage \\
\hline Stripped Low Locking 10 & 3847 & 200 & 5.509 & 4.987 & 40.106 & Breakage* \\
\hline Stripped Low Nonlocking 1 & 0 & & & & & \\
\hline Stripped Low Nonlocking 2 & 2868 & 100 & 8.947 & 8.722 & 11.465 & Pullout \\
\hline Stripped Low Nonlocking 3 & 1384 & 50 & 3.545 & 3.443 & 14.524 & Pullout \\
\hline Stripped Low Nonlocking 4 & 1261 & 50 & 3.729 & 3.627 & 13.784 & Pullout \\
\hline Stripped Low Nonlocking 5 & 2065 & 100 & 6.740 & 6.515 & 15.350 & Pullout \\
\hline Stripped Low Nonlocking 6 & 1202 & 50 & 4.089 & 3.987 & 12.542 & Pullout \\
\hline Stripped Low Nonlocking 7 & 546 & 25 & 2.116 & 2.070 & 12.078 & Pullout \\
\hline Stripped Low Nonlocking 8 & 716 & 25 & 1.774 & 1.728 & 14.467 & Pullout \\
\hline Stripped Low Nonlocking 9 & 1269 & 50 & 2.686 & 2.584 & 19.351 & Pullout \\
\hline Stripped Low Nonlocking 10 & 1609 & 50 & 2.877 & 2.775 & 18.020 & Pullout \\
\hline
\end{tabular}


Table XIX: CaP Cement Medium Density

\begin{tabular}{|c|c|c|c|c|c|c|}
\hline Trial & Cycles Completed & Load at failure $(\mathrm{N})$ & Avg displacement (mm) & Adjusted Displacement (mm) & Stiffness (N/mm) & Failure Mode \\
\hline Large Medium Locking 1 & 3830 & 200 & 4.219 & 3.696 & 54.112 & Pullout \\
\hline Large Medium Locking 2 & 4000 & 200 & 1.733 & 1.210 & 165.263 & Survived \\
\hline Large Medium Locking 3 & 4000 & 200 & 1.476 & 0.954 & 209.667 & Survived \\
\hline Large Medium Locking 4 & 4000 & 200 & 1.490 & 0.968 & 206.682 & Survived \\
\hline Large Medium Locking 5 & 4000 & 200 & 1.693 & 1.170 & 170.907 & Survived \\
\hline Large Medium Locking 6 & 4000 & 200 & 1.131 & 0.609 & 328.562 & Survived \\
\hline Large Medium Locking 7 & 4000 & 200 & 1.707 & 1.184 & 168.868 & Survived \\
\hline Large Medium Locking 8 & 4000 & 200 & 1.227 & 0.704 & 284.036 & Survived \\
\hline Large Medium Locking 9 & 4000 & 200 & 2.058 & 1.535 & 130.289 & Survived \\
\hline Large Medium Locking 10 & 4000 & 200 & 1.772 & 1.249 & 160.113 & Survived \\
\hline Large Medium Nonlocking 1 & 3139 & 200 & 8.207 & 7.685 & 26.025 & Breakage \\
\hline Large Medium Nonlocking 2 & 3202 & 200 & 7.234 & 6.711 & 29.802 & Breakage \\
\hline Large Medium Nonlocking 3 & 3160 & 200 & 7.612 & 7.090 & 28.210 & Breakage \\
\hline Large Medium Nonlocking 4 & 3220 & 200 & 6.346 & 5.824 & 34.343 & Breakage \\
\hline Large Medium Nonlocking 5 & 3167 & 200 & 7.425 & 6.903 & 28.975 & Breakage \\
\hline Large Medium Nonlocking 6 & 3103 & 200 & 7.491 & 6.968 & 28.701 & Breakage \\
\hline Large Medium Nonlocking 7 & - & - & - & - & - & - \\
\hline Large Medium Nonlocking 8 & - & - & - & - & - & - \\
\hline Large Medium Nonlocking 9 & - & - & - & - & - & - \\
\hline Large Medium Nonlocking 10 & - & - & - & - & - & - \\
\hline Stripped Medium Locking 1 & 1374 & 50 & 2.423 & 2.321 & 21.539 & Pullout \\
\hline Stripped Medium Locking 2 & 3081 & 200 & 2.984 & 2.461 & 81.267 & Pullout \\
\hline Stripped Medium Locking 3 & 3696 & 200 & 2.963 & 2.440 & 81.967 & Pullout \\
\hline Stripped Medium Locking 4 & 4000 & 200 & 2.322 & 1.799 & 111.178 & Survived \\
\hline Stripped Medium Locking 5 & 3143 & 200 & 3.636 & 3.114 & 64.229 & Pullout \\
\hline Stripped Medium Locking 6 & 3061 & 200 & 2.973 & 2.450 & 81.621 & Pullout \\
\hline Stripped Medium Locking 7 & 3926 & 200 & 3.530 & 3.008 & 66.495 & Pullout \\
\hline Stripped Medium Locking 8 & 3708 & 200 & 4.661 & 4.138 & 48.332 & Pullout \\
\hline Stripped Medium Locking 9 & 4000 & 200 & 3.022 & 2.499 & 80.023 & Survived \\
\hline Stripped Medium Locking 10 & 4000 & 200 & 3.282 & 2.759 & 72.481 & Survived \\
\hline Stripped Medium Nonlocking 1 & 2612 & 100 & 3.575 & 3.351 & 29.845 & Pullout \\
\hline Stripped Medium Nonlocking 2 & 3046 & 200 & 8.027 & 7.504 & 26.651 & Pullout \\
\hline Stripped Medium Nonlocking 3 & 3160 & 200 & 8.090 & 7.568 & 26.428 & Breakage \\
\hline Stripped Medium Nonlocking 4 & 3264 & 200 & 8.138 & 7.615 & 26.263 & Breakage \\
\hline Stripped Medium Nonlocking 5 & 3076 & 200 & 7.823 & 7.300 & 27.396 & Pullout \\
\hline Stripped Medium Nonlocking 6 & 3077 & 200 & 8.852 & 8.330 & 24.010 & Pullout \\
\hline Stripped Medium Nonlocking 7 & 3049 & 200 & 7.439 & 6.916 & 28.917 & Pullout \\
\hline Stripped Medium Nonlocking 8 & 3066 & 200 & 8.260 & 7.737 & 25.849 & Pullout \\
\hline Stripped Medium Nonlocking 9 & 3031 & 200 & 7.983 & 7.460 & 26.810 & Pullout \\
\hline Stripped Medium Nonlocking 10 & 2540 & 100 & 3.770 & 3.545 & 28.209 & Pullout \\
\hline
\end{tabular}


Table XX: CaP Cement High Density

\begin{tabular}{|c|c|c|c|c|c|c|}
\hline Trial & Cycles Completed & Load at failure $(\mathrm{N})$ & Avg displacement (mm) & Adjusted Displacement (mm) & Stiffness (N/mm) & Failure Mode \\
\hline Large High Locking 1 & 3075 & 200 & 4.911 & 4.388 & 45.578 & Pullout \\
\hline Large High Locking 2 & 2604 & 100 & 1.885 & 1.660 & 60.231 & Pullout \\
\hline Large High Locking 3 & 3234 & 200 & 5.030 & 4.507 & 44.376 & Pullout \\
\hline Large High Nonlocking 1 & 2130 & 100 & 6.440 & 6.215 & 16.090 & Pullout \\
\hline Large High Nonlocking 2 & 3090 & 200 & 7.794 & 7.272 & 27.503 & Pullout \\
\hline Large High Nonlocking 3 & 3153 & 200 & 8.573 & 8.050 & 24.844 & Breakage \\
\hline Stripped High Locking 1 & 3188 & 200 & 4.051 & 3.528 & 56.691 & Pullout \\
\hline Stripped High Locking 2 & 3005 & 200 & 4.143 & 3.620 & 55.245 & Pullout \\
\hline Stripped High Locking 3 & 3176 & 200 & 4.022 & 3.499 & 57.160 & Pullout \\
\hline Stripped High Locking 4 & 3371 & 200 & 3.832 & 3.310 & 60.432 & Pullout \\
\hline Stripped High Locking 5 & 4000 & 200 & 1.566 & 1.044 & 191.607 & Survived \\
\hline Stripped High Locking 6 & 4000 & 200 & 1.101 & 0.578 & 345.783 & Survived \\
\hline Stripped High Locking 7 & 4000 & 200 & 1.052 & 0.529 & 377.975 & Survived \\
\hline Stripped High Locking 8 & 2369 & 100 & 3.357 & 3.132 & 31.929 & Pullout \\
\hline Stripped High Locking 9 & 2639 & 100 & 3.504 & 3.280 & 30.490 & Pullout \\
\hline Stripped High Locking 10 & 2312 & 100 & 3.600 & 3.375 & 29.625 & Pullout \\
\hline Stripped High Nonlocking 1 & 3166 & 200 & 7.368 & 6.845 & 29.217 & Breakage \\
\hline Stripped High Nonlocking 2 & 1172 & 50 & 6.581 & 6.479 & 7.717 & Pullout \\
\hline Stripped High Nonlocking 3 & 1121 & 50 & 2.818 & 2.715 & 18.413 & Pullout \\
\hline Stripped High Nonlocking 4 & 3190 & 200 & 7.386 & 6.863 & 29.141 & Breakage \\
\hline Stripped High Nonlocking 5 & 3187 & 200 & 6.941 & 6.419 & 31.158 & Breakage \\
\hline Stripped High Nonlocking 6 & 45 & 25 & 0.666 & 0.620 & 40.306 & Pullout \\
\hline Stripped High Nonlocking 7 & 2378 & 100 & 6.318 & 6.093 & 16.413 & Pullout \\
\hline Stripped High Nonlocking 8 & 2275 & 100 & 6.918 & 6.693 & 14.940 & Pullout \\
\hline Stripped High Nonlocking 9 & 263 & 25 & 0.822 & 0.776 & 32.204 & Pullout \\
\hline Stripped High Nonlocking 10 & 2352 & 100 & 8.578 & 8.353 & 11.971 & Pullout \\
\hline
\end{tabular}


Table XXI: Displacement per Load Level Low Density Control

\begin{tabular}{|c|c|c|c|c|c|c|c|c|c|c|}
\hline \multirow[b]{2}{*}{ Trial } & \multirow[b]{2}{*}{ Recorded Load at failure (N) } & \multicolumn{4}{|c|}{ Averaged Displacement (mm) } & \multicolumn{4}{|c|}{ Adjusted Displacement (mm) } & \multirow[b]{2}{*}{ Failed? } \\
\hline & & $25 \mathrm{~N}$ & $50 \mathrm{~N}$ & $100 \mathrm{~N}$ & $200 \mathrm{~N}$ & $25 \mathrm{~N}$ & $50 \mathrm{~N}$ & $100 \mathrm{~N}$ & $200 \mathrm{~N}$ & \\
\hline Low Tight Locking 1 & 200 & 0.164 & 0.409 & 1.028 & 6.548 & 0.118 & 0.307 & 0.804 & 6.025 & Yes \\
\hline Low Tight Locking 2 & 200 & 0.207 & 0.435 & 1.058 & 6.828 & 0.161 & 0.333 & 0.833 & 6.306 & Yes \\
\hline Low Tight Locking 3 & 200 & 0.204 & 0.469 & 1.086 & 6.410 & 0.158 & 0.366 & 0.861 & 5.887 & Yes \\
\hline Low Tight Locking 4 & 200 & 0.253 & 0.524 & 1.083 & 5.565 & 0.207 & 0.421 & 0.858 & 5.042 & Yes \\
\hline Low Tight Locking 5 & 200 & 0.302 & 0.590 & 1.269 & 6.577 & 0.256 & 0.488 & 1.044 & 6.054 & Yes \\
\hline Low Tight Locking 6 & 200 & 0.239 & 0.537 & 2.930 & 15.892 & 0.193 & 0.435 & 2.705 & 15.370 & Yes \\
\hline Low Tight Locking 7 & 200 & 0.291 & 0.552 & 1.289 & 7.611 & 0.245 & 0.449 & 1.064 & 7.089 & Yes \\
\hline Low Tight Locking 8 & 200 & 0.240 & 0.491 & 1.185 & 8.666 & 0.194 & 0.389 & 0.960 & 8.143 & Yes \\
\hline Low Tight Locking 9 & 200 & 0.205 & 0.493 & 1.157 & 6.933 & 0.159 & 0.390 & 0.932 & 6.411 & Yes \\
\hline Low Tight Locking 10 & 200 & 0.315 & 0.599 & 1.288 & 7.271 & 0.269 & 0.497 & 1.063 & 6.749 & Yes \\
\hline Low Tight Nonlocking 1 & 200 & 0.232 & 0.648 & 3.420 & 19.770 & 0.186 & 0.545 & 3.195 & 19.247 & Yes \\
\hline Low Tight Nonlocking 2 & 200 & 0.204 & 0.347 & 1.815 & 17.759 & 0.158 & 0.244 & 1.591 & 17.237 & Yes \\
\hline Low Tight Nonlocking 3 & 200 & 0.235 & 0.670 & 3.655 & 18.815 & 0.189 & 0.568 & 3.430 & 18.292 & Yes \\
\hline Low Tight Nonlocking 4 & 200 & 0.189 & 0.392 & 8.151 & 18.130 & 0.143 & 0.290 & 7.926 & 17.608 & Yes \\
\hline Low Tight Nonlocking 5 & 200 & 0.238 & 0.644 & 2.417 & 18.672 & 0.192 & 0.542 & 2.193 & 18.149 & Yes \\
\hline Low Tight Nonlocking 6 & 100 & 0.310 & 1.061 & 16.557 & - & 0.264 & 0.959 & 16.332 & - & Yes \\
\hline Low Tight Nonlocking 7 & 100 & 0.305 & 0.991 & 16.335 & - & 0.259 & 0.889 & 16.110 & - & Yes \\
\hline Low Tight Nonlocking 8 & 100 & 0.197 & 0.436 & 15.624 & - & 0.151 & 0.334 & 15.400 & - & Yes \\
\hline Low Tight Nonlocking 9 & 200 & 0.182 & 0.348 & 1.696 & 17.787 & 0.136 & 0.246 & 1.471 & 17.265 & Yes \\
\hline Low Tight Nonlocking 10 & 200 & 0.225 & 0.596 & 2.701 & 20.703 & 0.179 & 0.494 & 2.477 & 20.181 & Yes \\
\hline Low Stripped Locking 1 & 100 & 0.322 & 1.491 & 3.495 & - & 0.276 & 1.389 & 3.270 & - & Yes \\
\hline Low Stripped Locking 2 & 200 & 0.450 & 1.415 & 3.438 & - & 0.404 & 1.313 & 3.213 & - & Yes \\
\hline Low Stripped Locking 3 & 200 & 0.291 & 1.314 & 3.184 & - & 0.245 & 1.212 & 2.959 & - & Yes \\
\hline Low Stripped Locking 4 & 100 & 0.607 & 2.215 & - & - & 0.561 & 2.113 & - & - & Yes \\
\hline Low Stripped Locking 5 & 50 & 0.664 & - & - & - & 0.618 & - & - & - & Yes \\
\hline Low Stripped Locking 6 & 25 & - & - & - & - & - & - & - & - & Yes \\
\hline Low Stripped Locking 7 & 50 & 0.716 & 2.771 & - & - & 0.670 & 2.669 & - & - & Yes \\
\hline Low Stripped Locking 8 & 200 & 0.309 & 1.142 & 3.170 & - & 0.263 & 1.040 & 2.945 & - & Yes \\
\hline Low Stripped Locking 9 & 50 & 0.501 & 3.842 & - & - & 0.455 & 3.740 & - & - & Yes \\
\hline Low Stripped Locking 10 & 25 & - & - & - & - & - & - & - & - & Yes \\
\hline Low Stripped Nonlocking 1 & 25 & 0.462 & - & - & - & 0.416 & - & - & - & Yes \\
\hline Low Stripped Nonlocking 2 & 25 & 0.686 & - & - & - & 0.640 & - & - & - & Yes \\
\hline Low Stripped Nonlocking 3 & 25 & 0.957 & - & - & - & 0.911 & - & - & - & Yes \\
\hline Low Stripped Nonlocking 4 & 25 & 0.734 & - & - & - & 0.688 & - & - & - & Yes \\
\hline Low Stripped Nonlocking 5 & 25 & 0.647 & - & - & - & 0.601 & - & - & - & Yes \\
\hline Low Stripped Nonlocking 6 & 25 & 1.265 & - & - & - & 1.219 & - & - & - & Yes \\
\hline Low Stripped Nonlocking 7 & 25 & 0.774 & - & - & - & 0.728 & - & - & - & Yes \\
\hline Low Stripped Nonlocking 8 & 25 & 1.000 & - & - & - & 0.954 & - & - & - & Yes \\
\hline Low Stripped Nonlocking 9 & 25 & 0.761 & - & - & - & 0.715 & - & - & - & Yes \\
\hline Low Stripped Nonlocking 10 & 25 & 1.058 & - & - & - & 1.012 & - & - & - & Yes \\
\hline
\end{tabular}


Table XXII: Displacement per Load Level Medium Density Control

\begin{tabular}{|c|c|c|c|c|c|c|c|c|c|c|}
\hline \multirow[b]{2}{*}{ Trial } & \multirow[b]{2}{*}{ Recorded Load at failure (N) } & \multicolumn{4}{|c|}{ Averaged Displacement (mm) } & \multicolumn{4}{|c|}{ Adjusted Displacement (mm) } & \multirow[b]{2}{*}{ Failed? } \\
\hline & & $25 \mathrm{~N}$ & $50 \mathrm{~N}$ & $100 \mathrm{~N}$ & $200 \mathrm{~N}$ & $25 \mathrm{~N}$ & $50 \mathrm{~N}$ & $100 \mathrm{~N}$ & $200 \mathrm{~N}$ & \\
\hline Medium Tight Locking 1 & 200 & 0.164 & 0.314 & 0.609 & 1.656 & 0.118 & 0.212 & 0.384 & 1.134 & No \\
\hline Medium Tight Locking 2 & 200 & 0.151 & 0.306 & 0.605 & 1.703 & 0.105 & 0.204 & 0.380 & 1.180 & No \\
\hline Medium Tight Locking 3 & 200 & 0.348 & 0.748 & 2.127 & 4.078 & 0.302 & 0.646 & 1.902 & 3.555 & Yes \\
\hline Medium Tight Locking 4 & 200 & 0.151 & 0.345 & 0.793 & 2.178 & 0.105 & 0.243 & 0.568 & 1.656 & No \\
\hline Medium Tight Locking 5 & 200 & 0.152 & 0.351 & 0.786 & 2.398 & 0.106 & 0.249 & 0.562 & 1.876 & No \\
\hline Medium Tight Locking 6 & 200 & 0.185 & 0.380 & 0.787 & 2.105 & 0.139 & 0.278 & 0.562 & 1.582 & No \\
\hline Medium Tight Locking 7 & 200 & 0.226 & 0.392 & 0.778 & 2.101 & 0.180 & 0.290 & 0.553 & 1.578 & No \\
\hline Medium Tight Locking 8 & 200 & 0.246 & 0.433 & 0.845 & 2.388 & 0.200 & 0.331 & 0.620 & 1.865 & No \\
\hline Medium Tight Locking 9 & 200 & 0.264 & 0.568 & 1.115 & 4.084 & 0.218 & 0.466 & 0.890 & 3.562 & Yes \\
\hline Medium Tight Locking 10 & 200 & 0.330 & 0.655 & 1.219 & 2.304 & 0.284 & 0.553 & 0.994 & 1.781 & No \\
\hline Medium Tight Nonlocking 1 & 200 & 0.184 & 0.308 & 1.191 & 7.795 & 0.138 & 0.206 & 0.966 & 7.273 & Yes \\
\hline Medium Tight Nonlocking 2 & 200 & 0.168 & 0.287 & 0.489 & 8.289 & 0.122 & 0.184 & 0.264 & 7.767 & Yes \\
\hline Medium Tight Nonlocking 3 & 200 & 0.200 & 0.591 & 1.554 & 7.842 & 0.155 & 0.489 & 1.330 & 7.320 & Yes \\
\hline Medium Tight Nonlocking 4 & 200 & 0.207 & 0.337 & 1.344 & 8.639 & 0.161 & 0.234 & 1.119 & 8.117 & Yes \\
\hline Medium Tight Nonlocking 5 & 200 & 0.150 & 0.283 & 0.683 & 8.037 & 0.104 & 0.181 & 0.458 & 7.514 & Yes \\
\hline Medium Tight Nonlocking 6 & 200 & 0.149 & 0.290 & 0.482 & 2.694 & 0.103 & 0.188 & 0.258 & 2.171 & No \\
\hline Medium Tight Nonlocking 7 & 200 & 0.174 & 0.305 & 0.534 & 4.742 & 0.128 & 0.202 & 0.310 & 4.220 & No \\
\hline Medium Tight Nonlocking 8 & 200 & 0.202 & 0.667 & 1.867 & 8.136 & 0.156 & 0.564 & 1.643 & 7.613 & Yes \\
\hline Medium Tight Nonlocking 9 & 200 & 0.268 & 0.721 & 1.781 & 7.800 & 0.222 & 0.619 & 1.556 & 7.277 & Yes \\
\hline Medium Tight Nonlocking 10 & 200 & 0.438 & 0.494 & 2.348 & 8.013 & 0.392 & 0.392 & 2.124 & 7.491 & Yes \\
\hline Medium Stripped Locking 1 & 200 & 0.308 & 1.011 & 3.212 & - & 0.262 & 0.909 & 2.988 & - & Yes \\
\hline Medium Stripped Locking 2 & 200 & 0.248 & 0.725 & 2.205 & 4.465 & 0.202 & 0.623 & 1.980 & 3.942 & Yes \\
\hline Medium Stripped Locking 3 & 200 & 0.205 & 0.632 & 1.751 & 4.356 & 0.159 & 0.530 & 1.527 & 3.833 & Yes \\
\hline Medium Stripped Locking 4 & 200 & 0.203 & 0.595 & 1.764 & 3.617 & 0.157 & 0.493 & 1.540 & 3.094 & Yes \\
\hline Medium Stripped Locking 5 & 200 & 0.248 & 0.734 & 1.800 & 3.848 & 0.202 & 0.632 & 1.575 & 3.325 & Yes \\
\hline Medium Stripped Locking 6 & 100 & 0.278 & 1.244 & 3.806 & - & 0.232 & 1.142 & 3.581 & - & Yes \\
\hline Medium Stripped Locking 7 & 200 & 0.277 & 0.768 & 1.757 & 4.708 & 0.231 & 0.666 & 1.532 & 4.185 & Yes \\
\hline Medium Stripped Locking 8 & 200 & 0.221 & 0.584 & 1.570 & 3.514 & 0.175 & 0.481 & 1.345 & 2.991 & Yes \\
\hline Medium Stripped Locking 9 & 200 & 0.214 & 0.597 & 1.613 & 4.073 & 0.168 & 0.495 & 1.388 & 3.550 & Yes \\
\hline Medium Stripped Locking 10 & 200 & 0.263 & 0.721 & 1.945 & 3.772 & 0.217 & 0.619 & 1.720 & 3.249 & Yes \\
\hline Medium Stripped Nonlocking 1 & 25 & 2.243 & - & - & - & 2.197 & - & - & - & Yes \\
\hline Medium Stripped Nonlocking 2 & 25 & 2.229 & - & - & - & 2.183 & - & - & - & Yes \\
\hline Medium Stripped Nonlocking 3 & 25 & 2.608 & - & - & - & 2.562 & - & - & - & Yes \\
\hline Medium Stripped Nonlocking 4 & 25 & 1.702 & - & - & - & 1.656 & - & - & - & Yes \\
\hline Medium Stripped Nonlocking 5 & 25 & 1.341 & - & - & - & 1.295 & - & - & - & Yes \\
\hline Medium Stripped Nonlocking 6 & 25 & 2.577 & - & - & - & 2.531 & - & - & - & Yes \\
\hline Medium Stripped Nonlocking 7 & 25 & 2.419 & - & - & - & 2.373 & - & - & - & Yes \\
\hline Medium Stripped Nonlocking 8 & 25 & 2.250 & - & - & - & 2.204 & - & - & - & Yes \\
\hline Medium Stripped Nonlocking 9 & 25 & 3.318 & - & - & - & 3.272 & - & - & - & Yes \\
\hline Medium Stripped Nonlocking 10 & 25 & 1.795 & - & - & - & 1.749 & - & - & - & Yes \\
\hline
\end{tabular}


Table XXIII: Displacement per Load Level High Density Control

\begin{tabular}{|c|c|c|c|c|c|c|c|c|c|c|}
\hline \multirow[b]{2}{*}{ Trial } & \multirow[b]{2}{*}{ Recorded Load at failure (N) } & \multicolumn{4}{|c|}{ Averaged Displacement (mm) } & \multicolumn{4}{|c|}{ Adjusted Displacement (mm) } & \multirow[b]{2}{*}{ Failed? } \\
\hline & & $25 \mathrm{~N}$ & $50 \mathrm{~N}$ & $100 \mathrm{~N}$ & $200 \mathrm{~N}$ & $25 \mathrm{~N}$ & $50 \mathrm{~N}$ & $100 \mathrm{~N}$ & $200 \mathrm{~N}$ & \\
\hline High Tight Locking 1 & 200 & 0.135 & 0.263 & 0.443 & 0.832 & 0.089 & 0.160 & 0.218 & 0.309 & No \\
\hline High Tight Locking 2 & 200 & 0.205 & 0.313 & 0.494 & 0.913 & 0.159 & 0.211 & 0.269 & 0.390 & No \\
\hline High Tight Locking 3 & 200 & 0.203 & 0.327 & 0.526 & 1.031 & 0.157 & 0.225 & 0.302 & 0.508 & No \\
\hline High Tight Locking 4 & 200 & 0.119 & 0.263 & 0.519 & 1.033 & 0.073 & 0.161 & 0.294 & 0.511 & No \\
\hline High Tight Locking 5 & 200 & 0.128 & 0.274 & 0.544 & 1.123 & 0.082 & 0.172 & 0.320 & 0.601 & No \\
\hline High Tight Locking 6 & 200 & 0.179 & 0.327 & 0.571 & 1.102 & 0.133 & 0.224 & 0.346 & 0.580 & No \\
\hline High Tight Locking 7 & 200 & 0.193 & 0.336 & 0.583 & 1.109 & 0.147 & 0.234 & 0.358 & 0.586 & No \\
\hline High Tight Locking 8 & 200 & 0.229 & 0.386 & 0.684 & 1.275 & 0.183 & 0.284 & 0.459 & 0.752 & No \\
\hline High Tight Locking 9 & 200 & 0.233 & 0.460 & 0.834 & 1.359 & 0.187 & 0.357 & 0.610 & 0.836 & No \\
\hline High Tight Locking 10 & 200 & 0.228 & 0.476 & 0.881 & 1.443 & 0.182 & 0.374 & 0.656 & 0.920 & No \\
\hline High Tight Nonlocking 1 & 200 & 0.069 & 0.136 & 0.300 & 0.740 & 0.023 & 0.034 & 0.075 & 0.217 & No \\
\hline High Tight Nonlocking 2 & 200 & 0.280 & 0.500 & 1.013 & 5.333 & 0.234 & 0.398 & 0.789 & 4.810 & Yes \\
\hline High Tight Nonlocking 3 & 200 & 0.226 & 0.455 & 0.997 & 5.313 & 0.180 & 0.353 & 0.772 & 4.791 & Yes \\
\hline High Tight Nonlocking 4 & 200 & 0.163 & 0.285 & 0.457 & 1.225 & 0.117 & 0.183 & 0.233 & 0.702 & No \\
\hline High Tight Nonlocking 5 & 200 & 0.182 & 0.299 & 0.590 & 1.673 & 0.136 & 0.197 & 0.366 & 1.150 & No \\
\hline High Tight Nonlocking 6 & 200 & 0.197 & 0.421 & 0.967 & 5.583 & 0.151 & 0.318 & 0.742 & 5.061 & Yes \\
\hline High Tight Nonlocking 7 & 200 & 0.228 & 0.416 & 0.864 & 2.065 & 0.182 & 0.314 & 0.640 & 1.543 & No \\
\hline High Tight Nonlocking 8 & 200 & 0.311 & 0.573 & 1.190 & 5.552 & 0.265 & 0.471 & 0.965 & 5.030 & Yes \\
\hline High Tight Nonlocking 9 & 200 & 0.315 & 0.566 & 1.144 & 5.600 & 0.269 & 0.464 & 0.919 & 5.078 & Yes \\
\hline High Tight Nonlocking 10 & 200 & 0.322 & 0.581 & 1.198 & 2.603 & 0.276 & 0.479 & 0.973 & 2.081 & No \\
\hline High Stripped Locking 1 & 100 & 0.200 & 0.771 & 3.594 & - & 0.155 & 0.669 & 3.370 & - & Yes \\
\hline High Stripped Locking 2 & 50 & 0.356 & 2.634 & - & - & 0.310 & 2.531 & - & - & Yes \\
\hline High Stripped Locking 3 & 50 & 0.256 & 3.344 & - & - & 0.210 & 3.242 & - & - & Yes \\
\hline High Stripped Locking 4 & 50 & 0.313 & 2.832 & - & - & 0.267 & 2.730 & - & - & Yes \\
\hline High Stripped Locking 5 & 50 & 0.775 & - & - & - & 0.729 & - & - & - & Yes \\
\hline High Stripped Locking 6 & 50 & 0.282 & 2.805 & - & - & 0.236 & 2.703 & - & - & Yes \\
\hline High Stripped Locking 7 & 25 & 0.856 & - & - & - & 0.810 & - & - & - & Yes \\
\hline High Stripped Locking 8 & 100 & 0.254 & 2.382 & - & - & 0.208 & 2.280 & - & - & Yes \\
\hline High Stripped Locking 9 & 50 & 0.338 & 3.335 & - & - & 0.292 & 3.233 & - & - & Yes \\
\hline High Stripped Locking 10 & 50 & 0.357 & 4.460 & - & - & 0.311 & 4.358 & - & - & Yes \\
\hline High Stripped Nonlocking 1 & 25 & 1.161 & - & - & - & 1.115 & - & - & - & Yes \\
\hline High Stripped Nonlocking 2 & 25 & 2.856 & - & - & - & 2.810 & - & - & - & Yes \\
\hline High Stripped Nonlocking 3 & 25 & 1.743 & - & - & - & 1.697 & - & - & - & Yes \\
\hline High Stripped Nonlocking 4 & 25 & 1.647 & - & - & - & 1.601 & - & - & - & Yes \\
\hline High Stripped Nonlocking 5 & 25 & 2.223 & - & - & - & 2.177 & - & - & - & Yes \\
\hline High Stripped Nonlocking 6 & 25 & 2.512 & - & - & - & 2.466 & - & - & - & Yes \\
\hline High Stripped Nonlocking 7 & 25 & 2.653 & - & - & - & 2.607 & - & - & - & Yes \\
\hline High Stripped Nonlocking 8 & 25 & 2.657 & - & - & - & 2.611 & - & - & - & Yes \\
\hline High Stripped Nonlocking 9 & 25 & 2.333 & - & - & - & 2.287 & - & - & - & Yes \\
\hline High Stripped Nonlocking 10 & 25 & 2.347 & - & - & - & 2.301 & - & - & - & Yes \\
\hline
\end{tabular}


Table XXIV: Displacement per Load Level Low Density PMMA

\begin{tabular}{|c|c|c|c|c|c|c|c|c|c|c|}
\hline \multirow[b]{2}{*}{ Trial } & \multirow[b]{2}{*}{ Recorded Load at failure (N) } & \multicolumn{4}{|c|}{ Averaged Displacement (mm) } & \multicolumn{4}{|c|}{ Adjusted Displacement (mm) } & \multirow[b]{2}{*}{ Failed? } \\
\hline & & $25 \mathrm{~N}$ & $50 \mathrm{~N}$ & $100 \mathrm{~N}$ & $200 \mathrm{~N}$ & $25 \mathrm{~N}$ & $50 \mathrm{~N}$ & $100 \mathrm{~N}$ & $200 \mathrm{~N}$ & \\
\hline Large Low Locking 1 & 200 & 0.115 & 0.259 & 0.528 & 1.003 & 0.069 & 0.157 & 0.304 & 0.480 & No \\
\hline Large Low Locking 2 & 200 & 0.105 & 0.230 & 0.488 & 1.083 & 0.059 & 0.128 & 0.264 & 0.560 & No \\
\hline Large Low Locking 3 & 200 & 0.128 & 0.264 & 0.550 & 1.085 & 0.082 & 0.162 & 0.326 & 0.562 & No \\
\hline Large Low Locking 4 & 200 & 0.140 & 0.300 & 0.702 & 4.666 & 0.094 & 0.198 & 0.477 & 4.144 & Yes \\
\hline Large Low Locking 5 & 200 & 0.339 & 0.603 & 0.957 & 1.775 & 0.293 & 0.500 & 0.732 & 1.253 & No \\
\hline Large Low Locking 6 & 200 & 0.104 & 0.217 & 0.503 & 1.019 & 0.058 & 0.115 & 0.278 & 0.497 & No \\
\hline Large Low Locking 7 & 200 & 0.221 & 0.414 & 0.712 & 1.274 & 0.175 & 0.312 & 0.487 & 0.751 & No \\
\hline Large Low Locking 8 & 200 & 0.204 & 0.411 & 0.722 & 1.331 & 0.158 & 0.308 & 0.498 & 0.808 & No \\
\hline Large Low Locking 9 & 200 & 0.163 & 0.345 & 0.688 & 1.404 & 0.117 & 0.243 & 0.464 & 0.882 & No \\
\hline Large Low Locking 10 & 200 & 0.460 & 1.027 & 0.653 & 1.218 & 0.414 & 0.925 & 0.429 & 0.696 & No \\
\hline Large Low Nonlocking 1 & 200 & 0.287 & 0.473 & 0.782 & 5.150 & 0.241 & 0.371 & 0.557 & 4.627 & Yes \\
\hline Large Low Nonlocking 2 & 200 & 0.296 & 0.500 & 0.948 & 1.885 & 0.250 & 0.398 & 0.723 & 1.362 & No \\
\hline Large Low Nonlocking 3 & 200 & 0.302 & 0.504 & 0.930 & 1.853 & 0.256 & 0.402 & 0.705 & 1.331 & No \\
\hline Large Low Nonlocking 4 & 200 & 0.334 & 0.581 & 1.084 & 5.289 & 0.288 & 0.479 & 0.859 & 4.766 & Yes \\
\hline Large Low Nonlocking 5 & 200 & 0.316 & 0.548 & 1.032 & 5.383 & 0.270 & 0.446 & 0.808 & 4.861 & Yes \\
\hline Large Low Nonlocking 6 & 200 & 0.435 & 0.719 & 1.223 & 5.137 & 0.389 & 0.617 & 0.998 & 4.614 & Yes \\
\hline Large Low Nonlocking 7 & 200 & 0.290 & 0.505 & 0.933 & 5.082 & 0.244 & 0.403 & 0.708 & 4.560 & Yes \\
\hline Large Low Nonlocking 8 & 200 & 0.296 & 0.516 & 1.008 & 4.680 & 0.250 & 0.414 & 0.783 & 4.157 & Yes \\
\hline Large Low Nonlocking 9 & 200 & 0.426 & 0.784 & 1.543 & 6.534 & 0.380 & 0.682 & 1.319 & 6.012 & Yes \\
\hline Large Low Nonlocking 10 & 200 & 0.494 & 0.982 & 2.217 & 7.481 & 0.448 & 0.880 & 1.993 & 6.958 & Yes \\
\hline Stripped Low Locking 1 & 200 & 0.147 & 0.310 & 0.670 & 1.964 & 0.101 & 0.208 & 0.446 & 1.441 & No \\
\hline Stripped Low Locking 2 & 200 & 0.175 & 0.368 & 0.740 & 1.655 & 0.129 & 0.266 & 0.516 & 1.132 & No \\
\hline Stripped Low Locking 3 & 200 & 0.206 & 0.484 & 1.182 & 2.413 & 0.160 & 0.382 & 0.958 & 1.890 & No \\
\hline Stripped Low Locking 4 & 200 & 0.211 & 0.440 & 0.871 & 2.007 & 0.165 & 0.337 & 0.646 & 1.485 & No \\
\hline Stripped Low Locking 5 & 200 & 0.219 & 0.437 & 0.895 & 8.110 & 0.173 & 0.335 & 0.671 & 7.587 & Yes \\
\hline Stripped Low Locking 6 & 200 & 0.186 & 0.390 & 0.874 & 1.757 & 0.140 & 0.287 & 0.650 & 1.235 & No \\
\hline Stripped Low Locking 7 & 200 & 0.177 & 0.384 & 0.824 & 1.832 & 0.131 & 0.282 & 0.599 & 1.309 & No \\
\hline Stripped Low Locking 8 & 200 & 0.178 & 0.406 & 0.810 & 1.800 & 0.132 & 0.304 & 0.585 & 1.277 & No \\
\hline Stripped Low Locking 9 & 200 & 0.170 & 0.390 & 0.775 & 1.531 & 0.124 & 0.288 & 0.550 & 1.009 & No \\
\hline Stripped Low Locking 10 & 200 & 0.326 & 0.606 & 1.030 & 1.862 & 0.280 & 0.504 & 0.805 & 1.340 & No \\
\hline Stripped Low Nonlocking 1 & 200 & 0.193 & 0.347 & 2.337 & 10.312 & 0.147 & 0.245 & 2.112 & 9.790 & Yes \\
\hline Stripped Low Nonlocking 2 & 200 & 0.189 & 0.733 & 1.805 & 11.251 & 0.143 & 0.631 & 1.580 & 10.728 & Yes \\
\hline Stripped Low Nonlocking 3 & 100 & 0.661 & 1.678 & 3.437 & - & 0.615 & 1.576 & 3.213 & - & Yes \\
\hline Stripped Low Nonlocking 4 & 200 & 0.469 & 0.974 & 1.909 & 7.578 & 0.423 & 0.871 & 1.684 & 7.055 & Yes \\
\hline Stripped Low Nonlocking 5 & 200 & 0.404 & 1.838 & 3.953 & - & 0.358 & 1.736 & 3.728 & - & Yes \\
\hline Stripped Low Nonlocking 6 & 200 & 0.503 & 1.133 & 2.239 & 7.582 & 0.457 & 1.031 & 2.014 & 7.060 & Yes \\
\hline Stripped Low Nonlocking 7 & 200 & 0.415 & 1.213 & 3.132 & - & 0.369 & 1.111 & 2.907 & - & Yes \\
\hline Stripped Low Nonlocking 8 & 200 & 0.428 & 1.022 & 2.165 & 6.594 & 0.382 & 0.920 & 1.940 & 6.071 & Yes \\
\hline Stripped Low Nonlocking 9 & 200 & 0.272 & 0.753 & 1.610 & 6.856 & 0.227 & 0.651 & 1.386 & 6.333 & Yes \\
\hline Stripped Low Nonlocking 10 & 200 & 0.533 & 0.775 & 1.422 & 5.774 & 0.487 & 0.673 & 1.197 & 5.252 & Yes \\
\hline
\end{tabular}


Table XXV: Displacement per Load Level Medium Density PMMA

\begin{tabular}{|c|c|c|c|c|c|c|c|c|c|c|}
\hline \multirow[b]{2}{*}{ Trial } & \multirow[b]{2}{*}{ Recorded Load at failure (N) } & \multicolumn{4}{|c|}{ Averaged Displacement (mm) } & \multicolumn{4}{|c|}{ Adjusted Displacement (mm) } & \multirow[b]{2}{*}{ Failed? } \\
\hline & & $25 \mathrm{~N}$ & $50 \mathrm{~N}$ & $100 \mathrm{~N}$ & $200 \mathrm{~N}$ & $25 \mathrm{~N}$ & $50 \mathrm{~N}$ & $100 \mathrm{~N}$ & $200 \mathrm{~N}$ & \\
\hline Stripped Medium Locking 1 & 200 & 0.242 & 0.521 & 0.905 & 1.219 & 0.196 & 0.419 & 0.680 & 0.696 & No \\
\hline Stripped Medium Locking 2 & 200 & 0.257 & 0.489 & 0.764 & 1.321 & 0.211 & 0.387 & 0.539 & 0.799 & No \\
\hline Stripped Medium Locking 3 & 200 & 0.171 & 0.352 & 0.636 & 1.203 & 0.125 & 0.250 & 0.411 & 0.680 & No \\
\hline Stripped Medium Locking 4 & 200 & 0.178 & 0.461 & 0.744 & 1.357 & 0.132 & 0.359 & 0.519 & 0.834 & No \\
\hline Stripped Medium Locking 5 & 200 & 0.158 & 0.366 & 0.630 & 1.178 & 0.112 & 0.264 & 0.405 & 0.656 & No \\
\hline Stripped Medium Locking 6 & 200 & 0.133 & 0.318 & 0.603 & 1.208 & 0.087 & 0.216 & 0.378 & 0.686 & No \\
\hline Stripped Medium Locking 7 & 50 & 0.239 & 2.809 & - & - & 0.193 & 2.706 & - & - & Yes \\
\hline Stripped Medium Locking 8 & 200 & 0.165 & 0.322 & 0.548 & 0.992 & 0.119 & 0.220 & 0.323 & 0.469 & No \\
\hline Stripped Medium Locking 9 & 200 & 0.152 & 0.298 & 0.566 & 1.745 & 0.106 & 0.196 & 0.341 & 1.223 & No \\
\hline Stripped Medium Locking 10 & 200 & 0.213 & 0.442 & 0.820 & 1.447 & 0.167 & 0.339 & 0.595 & 0.924 & No \\
\hline Stripped Medium Nonlocking 1 & 100 & 0.187 & 0.793 & 2.758 & - & 0.141 & 0.691 & 2.533 & - & Yes \\
\hline Stripped Medium Nonlocking 2 & 25 & 1.297 & - & - & - & 1.251 & - & - & - & Yes \\
\hline Stripped Medium Nonlocking 3 & 50 & 0.408 & 1.555 & - & - & 0.362 & 1.453 & - & - & Yes \\
\hline Stripped Medium Nonlocking 4 & 200 & 0.396 & 1.030 & 2.787 & - & 0.350 & 0.928 & 2.562 & - & Yes \\
\hline Stripped Medium Nonlocking 5 & 25 & 0.523 & - & - & - & 0.477 & - & - & - & Yes \\
\hline Stripped Medium Nonlocking 6 & 200 & 0.357 & 0.843 & 2.060 & 12.084 & 0.311 & 0.741 & 1.835 & 11.561 & Yes \\
\hline Stripped Medium Nonlocking 7 & 100 & 0.531 & 1.311 & 3.995 & - & 0.485 & 1.209 & 3.770 & - & Yes \\
\hline Stripped Medium Nonlocking 8 & 50 & 0.440 & 1.767 & - & - & 0.394 & 1.665 & - & - & Yes \\
\hline Stripped Medium Nonlocking 9 & 200 & 0.562 & 1.048 & 1.858 & 7.392 & 0.516 & 0.945 & 1.633 & 6.869 & Yes \\
\hline Stripped Medium Nonlocking 10 & 200 & 0.286 & 0.420 & 1.105 & 3.908 & 0.240 & 0.318 & 0.880 & 3.385 & No \\
\hline Large Medium Locking 1 & 200 & 0.085 & 0.183 & 0.381 & 0.741 & 0.039 & 0.081 & 0.156 & 0.219 & No \\
\hline Large Medium Locking 2 & 200 & 0.192 & 0.278 & 0.436 & 0.762 & 0.146 & 0.176 & 0.211 & 0.240 & No \\
\hline Large Medium Locking 3 & 200 & 0.200 & 0.301 & 0.483 & 0.848 & 0.155 & 0.199 & 0.258 & 0.326 & No \\
\hline Large Medium Locking 4 & 200 & 0.106 & 0.239 & 0.447 & 0.837 & 0.060 & 0.137 & 0.222 & 0.315 & No \\
\hline Large Medium Locking 5 & 200 & 0.071 & 0.184 & 0.382 & 0.744 & 0.025 & 0.082 & 0.157 & 0.222 & No \\
\hline Large Medium Locking 6 & 200 & 0.111 & 0.282 & 0.599 & 0.988 & 0.065 & 0.180 & 0.375 & 0.465 & No \\
\hline Large Medium Locking 7 & 200 & 0.116 & 0.251 & 0.454 & 0.888 & 0.070 & 0.149 & 0.230 & 0.366 & No \\
\hline Large Medium Locking 8 & 200 & 0.166 & 0.269 & 0.462 & 0.832 & 0.120 & 0.167 & 0.237 & 0.310 & No \\
\hline Large Medium Locking 9 & 200 & 0.205 & 0.330 & 0.542 & 0.967 & 0.159 & 0.228 & 0.317 & 0.444 & No \\
\hline Large Medium Locking 10 & 200 & 0.228 & 0.342 & 0.549 & 0.994 & 0.182 & 0.240 & 0.324 & 0.472 & No \\
\hline Large Medium Nonlocking 1 & 200 & 0.234 & 0.419 & 0.768 & 4.356 & 0.188 & 0.317 & 0.543 & 3.834 & Yes \\
\hline Large Medium Nonlocking 2 & 200 & 0.240 & 0.392 & 0.728 & 1.486 & 0.194 & 0.290 & 0.503 & 0.963 & No \\
\hline Large Medium Nonlocking 3 & 200 & 0.255 & 0.437 & 0.815 & 4.701 & 0.209 & 0.335 & 0.590 & 4.178 & Yes \\
\hline Large Medium Nonlocking 4 & 200 & 0.244 & 0.417 & 0.774 & 4.292 & 0.198 & 0.315 & 0.549 & 3.769 & Yes \\
\hline Large Medium Nonlocking 5 & 200 & 0.249 & 0.422 & 0.758 & 4.333 & 0.203 & 0.320 & 0.533 & 3.811 & Yes \\
\hline Large Medium Nonlocking 6 & 200 & 0.237 & 0.395 & 0.704 & 1.351 & 0.191 & 0.293 & 0.480 & 0.828 & No \\
\hline Large Medium Nonlocking 7 & 200 & 0.245 & 0.419 & 0.792 & 1.640 & 0.199 & 0.317 & 0.567 & 1.118 & No \\
\hline Large Medium Nonlocking 8 & 200 & 0.248 & 0.437 & 0.803 & 4.595 & 0.202 & 0.335 & 0.578 & 4.073 & Yes \\
\hline Large Medium Nonlocking 9 & 200 & 0.267 & 0.472 & 0.832 & 4.582 & 0.221 & 0.370 & 0.608 & 4.059 & Yes \\
\hline Large Medium Nonlocking 10 & 200 & 0.257 & 0.459 & 0.823 & 1.811 & 0.211 & 0.357 & 0.598 & 1.289 & No \\
\hline
\end{tabular}


Table XXVI: Displacement per Load Level High Density PMMA

\begin{tabular}{|c|c|c|c|c|c|c|c|c|c|c|}
\hline \multirow[b]{2}{*}{ Trial } & \multirow[b]{2}{*}{ Recorded Load at failure (N) } & \multicolumn{4}{|c|}{ Averaged Displacement (mm) } & \multicolumn{4}{|c|}{ Adjusted Displacement (mm) } & \multirow[b]{2}{*}{ Failed? } \\
\hline & & $25 \mathrm{~N}$ & $50 \mathrm{~N}$ & $100 \mathrm{~N}$ & $200 \mathrm{~N}$ & $25 \mathrm{~N}$ & $50 \mathrm{~N}$ & $100 \mathrm{~N}$ & $200 \mathrm{~N}$ & \\
\hline Stripped High Locking 1 & 200 & 0.239 & 0.440 & 0.828 & 1.495 & 0.193 & 0.338 & 0.604 & 0.972 & No \\
\hline Stripped High Locking 2 & 200 & 0.176 & 0.383 & 0.670 & 1.551 & 0.130 & 0.281 & 0.445 & 1.028 & No \\
\hline Stripped High Locking 3 & 200 & 0.141 & 0.330 & 0.608 & 1.244 & 0.095 & 0.228 & 0.383 & 0.722 & No \\
\hline Stripped High Locking 4 & 200 & 0.147 & 0.319 & 0.558 & 1.060 & 0.101 & 0.217 & 0.334 & 0.537 & No \\
\hline Stripped High Locking 5 & 200 & 0.143 & 0.319 & 0.600 & 1.206 & 0.097 & 0.217 & 0.375 & 0.684 & No \\
\hline Stripped High Locking 6 & 200 & 0.142 & 0.309 & 0.569 & 1.150 & 0.096 & 0.207 & 0.344 & 0.627 & No \\
\hline Stripped High Locking 7 & 200 & 0.153 & 0.344 & 0.649 & 1.380 & 0.107 & 0.242 & 0.424 & 0.857 & No \\
\hline Stripped High Locking 8 & 200 & 0.138 & 0.295 & 0.583 & 1.276 & 0.092 & 0.192 & 0.358 & 0.753 & No \\
\hline Stripped High Locking 9 & 200 & 0.127 & 0.266 & 0.552 & 1.175 & 0.081 & 0.164 & 0.327 & 0.652 & No \\
\hline Stripped High Locking 10 & 200 & 0.138 & 0.302 & 0.608 & 1.304 & 0.092 & 0.200 & 0.383 & 0.781 & No \\
\hline Stripped High Nonlocking 1 & 200 & 0.264 & 0.495 & 1.018 & 5.792 & 0.218 & 0.393 & 0.793 & 5.269 & Yes \\
\hline Stripped High Nonlocking 2 & 200 & 0.234 & 0.467 & 0.901 & 4.968 & 0.188 & 0.365 & 0.676 & 4.446 & Yes \\
\hline Stripped High Nonlocking 3 & 200 & 0.327 & 0.512 & 0.910 & 2.110 & 0.281 & 0.410 & 0.686 & 1.587 & No \\
\hline Stripped High Nonlocking 4 & 200 & 0.301 & 0.591 & 1.212 & 6.383 & 0.255 & 0.489 & 0.987 & 5.861 & Yes \\
\hline Stripped High Nonlocking 5 & 200 & 0.433 & 0.772 & 1.447 & 6.412 & 0.387 & 0.670 & 1.222 & 5.890 & Yes \\
\hline Stripped High Nonlocking 6 & 200 & 0.417 & 0.773 & 1.445 & 6.515 & 0.371 & 0.671 & 1.220 & 5.993 & Yes \\
\hline Stripped High Nonlocking 7 & 200 & 0.496 & 0.873 & 1.613 & 6.714 & 0.450 & 0.771 & 1.388 & 6.192 & Yes \\
\hline Stripped High Nonlocking 8 & 200 & 0.280 & 0.490 & 0.946 & 2.509 & 0.234 & 0.387 & 0.722 & 1.986 & No \\
\hline Stripped High Nonlocking 9 & 200 & 0.406 & 0.711 & 1.185 & 2.575 & 0.360 & 0.609 & 0.960 & 2.053 & No \\
\hline Stripped High Nonlocking 10 & 200 & 0.552 & 1.038 & 1.959 & 7.158 & 0.506 & 0.936 & 1.735 & 6.635 & Yes \\
\hline Large High Locking 1 & 200 & 0.186 & 0.333 & 0.544 & 0.923 & 0.140 & 0.231 & 0.319 & 0.400 & No \\
\hline Large High Locking 2 & 200 & 0.098 & 0.212 & 0.448 & 0.852 & 0.052 & 0.110 & 0.223 & 0.330 & No \\
\hline Large High Locking 3 & 200 & 0.088 & 0.201 & 0.433 & 0.835 & 0.042 & 0.099 & 0.208 & 0.312 & No \\
\hline Large High Locking 4 & 200 & 0.209 & 0.315 & 0.504 & 0.886 & 0.163 & 0.213 & 0.279 & 0.364 & No \\
\hline Large High Locking 5 & 200 & 0.199 & 0.412 & 0.747 & 1.006 & 0.153 & 0.310 & 0.522 & 0.483 & No \\
\hline Large High Locking 6 & 200 & 0.215 & 0.427 & 0.799 & 1.293 & 0.169 & 0.325 & 0.574 & 0.771 & No \\
\hline Large High Locking 7 & 200 & 0.074 & 0.173 & 0.385 & 0.772 & 0.028 & 0.071 & 0.160 & 0.249 & No \\
\hline Large High Locking 8 & 200 & 0.115 & 0.241 & 0.424 & 0.789 & 0.069 & 0.139 & 0.200 & 0.266 & No \\
\hline Large High Locking 9 & 200 & 0.207 & 0.316 & 0.497 & 0.875 & 0.161 & 0.214 & 0.272 & 0.352 & No \\
\hline Large High Locking 10 & 200 & 0.224 & 0.327 & 0.522 & 0.932 & 0.178 & 0.224 & 0.297 & 0.409 & No \\
\hline Large High Nonlocking 1 & 200 & 0.243 & 0.392 & 0.690 & 1.575 & 0.197 & 0.290 & 0.466 & 1.052 & No \\
\hline Large High Nonlocking 2 & 200 & 0.253 & 0.412 & 0.758 & 1.531 & 0.207 & 0.310 & 0.533 & 1.008 & No \\
\hline Large High Nonlocking 3 & 200 & 0.263 & 0.425 & 0.778 & 5.021 & 0.217 & 0.323 & 0.553 & 4.499 & Yes \\
\hline Large High Nonlocking 4 & 200 & 0.260 & 0.417 & 0.742 & 1.610 & 0.214 & 0.315 & 0.518 & 1.088 & No \\
\hline Large High Nonlocking 5 & 200 & 0.328 & 0.570 & 1.014 & 4.709 & 0.282 & 0.468 & 0.789 & 4.187 & Yes \\
\hline Large High Nonlocking 6 & 200 & 0.264 & 0.426 & 0.762 & 5.020 & 0.218 & 0.324 & 0.537 & 4.497 & Yes \\
\hline Large High Nonlocking 7 & 200 & 0.254 & 0.394 & 0.696 & 1.435 & 0.208 & 0.292 & 0.471 & 0.913 & No \\
\hline Large High Nonlocking 8 & 200 & 0.261 & 0.435 & 0.790 & 4.541 & 0.215 & 0.333 & 0.565 & 4.018 & Yes \\
\hline Large High Nonlocking 9 & 200 & 0.256 & 0.422 & 0.745 & 1.533 & 0.210 & 0.320 & 0.520 & 1.011 & No \\
\hline Large High Nonlocking 10 & 200 & 0.248 & 0.405 & 0.727 & 4.405 & 0.202 & 0.303 & 0.502 & 3.882 & Yes \\
\hline
\end{tabular}


Table XXVII: Displacement per Load Level Low Density CaP

\begin{tabular}{|c|c|c|c|c|c|c|c|c|c|c|}
\hline \multirow[b]{2}{*}{ Trial } & \multirow[b]{2}{*}{ Load at failure (N) } & \multicolumn{4}{|c|}{ Averaged Displacement (mm) } & \multicolumn{4}{|c|}{ Adjusted Displacement (mm) } & \multirow[b]{2}{*}{ Failed? } \\
\hline & & $25 \mathrm{~N}$ & $50 \mathrm{~N}$ & $100 \mathrm{~N}$ & $200 \mathrm{~N}$ & $25 \mathrm{~N}$ & $50 \mathrm{~N}$ & $100 \mathrm{~N}$ & $200 \mathrm{~N}$ & \\
\hline RETEST Large Low Locking 1 & 200 & 0.233 & 0.374 & 0.747 & 2.236 & 0.187 & 0.272 & 0.522 & 1.713 & No \\
\hline RETEST Large Low Locking 2 & 200 & 0.140 & 0.339 & 0.681 & 1.616 & 0.094 & 0.237 & 0.456 & 1.094 & No \\
\hline RETEST Large Low Locking 3 & 200 & 0.214 & 0.421 & 0.854 & 1.948 & 0.168 & 0.319 & 0.630 & 1.425 & No \\
\hline RETEST Large Low Locking 4 & 200 & 0.281 & 0.508 & 1.081 & 4.292 & 0.235 & 0.406 & 0.857 & 3.769 & Yes \\
\hline RETEST Large Low Locking 5 & 200 & 0.220 & 0.415 & 0.822 & 1.504 & 0.174 & 0.313 & 0.598 & 0.981 & No \\
\hline RETEST Large Low Locking 6 & 200 & 0.424 & 0.697 & 1.101 & 1.844 & 0.378 & 0.595 & 0.876 & 1.322 & No \\
\hline TEST Large Low Locking 7 & 200 & 0.286 & 0.485 & 0.861 & 1.933 & 0.240 & 0.383 & 0.636 & 1.411 & No \\
\hline TEST Large Low Locking 8 & 200 & 0.187 & 0.422 & 0.920 & 3.286 & 0.141 & 0.320 & 0.696 & 2.763 & Yes \\
\hline TEST Large Low Locking 9 & 200 & 0.317 & 0.511 & 0.868 & 1.675 & 0.271 & 0.409 & 0.644 & 1.152 & No \\
\hline TEST Large Low Locking 10 & 200 & 0.252 & 0.431 & 0.723 & 1.394 & 0.206 & 0.329 & 0.498 & 0.872 & No \\
\hline RETEST Large Low Nonlocking 1 & 200 & 0.391 & 0.848 & 1.815 & 7.504 & 0.345 & 0.746 & 1.591 & 6.981 & Yes \\
\hline RETEST Large Low Nonlocking 2 & 200 & 0.366 & 0.683 & 1.433 & 7.088 & 0.320 & 0.581 & 1.208 & 6.565 & Yes \\
\hline RETEST Large Low Nonlocking 3 & 200 & 0.410 & 0.898 & 1.684 & 7.479 & 0.364 & 0.796 & 1.459 & 6.957 & Yes \\
\hline RETEST Large Low Nonlocking 4 & 200 & 0.520 & 0.971 & 2.005 & 7.809 & 0.474 & 0.869 & 1.780 & 7.286 & Yes \\
\hline RETEST Large Low Nonlocking 5 & 200 & 0.475 & 0.916 & 4.388 & 9.268 & 0.429 & 0.814 & 4.163 & 8.745 & Yes \\
\hline RETEST Large Low Nonlocking 6 & 200 & 0.666 & 1.266 & 2.800 & 8.208 & 0.620 & 1.164 & 2.576 & 7.685 & Yes \\
\hline TEST Large Low Nonlocking 7 & 200 & 0.302 & 0.660 & 1.606 & 7.661 & 0.256 & 0.558 & 1.382 & 7.138 & Yes \\
\hline TEST Large Low Nonlocking 8 & 200 & 0.486 & 1.023 & 2.062 & 10.197 & 0.440 & 0.921 & 1.837 & 9.675 & Yes \\
\hline TEST Large Low Nonlocking 9 & 200 & 0.265 & 0.749 & 2.210 & 8.711 & 0.219 & 0.647 & 1.985 & 8.189 & Yes \\
\hline TEST Large Low Nonlocking 10 & 200 & 0.472 & 0.805 & 2.148 & 10.455 & 0.426 & 0.703 & 1.923 & 9.932 & Yes \\
\hline Stripped Low Locking 1 & 200 & 0.263 & 0.494 & 1.115 & 4.623 & 0.217 & 0.392 & 0.890 & 4.100 & Yes \\
\hline Stripped Low Locking 2 & 200 & 0.297 & 0.549 & 1.116 & 3.631 & 0.251 & 0.447 & 0.892 & 3.108 & Yes \\
\hline Stripped Low Locking 3 & 200 & 0.270 & 0.507 & 1.068 & 3.550 & 0.224 & 0.405 & 0.843 & 3.028 & Yes \\
\hline Stripped Low Locking 4 & 100 & 0.339 & 0.618 & 2.017 & - & 0.293 & 0.516 & 1.792 & - & Yes \\
\hline Stripped Low Locking 5 & 200 & 0.375 & 0.735 & 1.321 & 4.040 & 0.330 & 0.633 & 1.097 & 3.518 & Yes \\
\hline Stripped Low Locking 6 & 200 & 0.225 & 0.487 & 1.212 & 3.815 & 0.179 & 0.385 & 0.987 & 3.292 & Yes \\
\hline Stripped Low Locking 7 & 200 & 0.339 & 0.735 & 1.301 & 3.429 & 0.293 & 0.633 & 1.076 & 2.906 & Yes \\
\hline Stripped Low Locking 8 & 100 & 0.422 & 0.939 & 2.362 & - & 0.376 & 0.837 & 2.137 & - & Yes \\
\hline Stripped Low Locking 9 & 200 & 0.268 & 0.486 & 1.218 & 5.570 & 0.222 & 0.384 & 0.993 & 5.047 & Yes \\
\hline Stripped Low Locking 10 & 200 & 0.313 & 0.792 & 1.506 & 5.509 & 0.267 & 0.690 & 1.281 & 4.987 & Yes \\
\hline Stripped Low Nonlocking $1^{*}$ & - & - & - & - & - & - & - & - & - & - \\
\hline Stripped Low Nonlocking 2 & 100 & 0.757 & 1.346 & 8.947 & - & 0.711 & 1.244 & 8.722 & - & Yes \\
\hline Stripped Low Nonlocking 3 & 50 & 0.928 & 3.545 & - & - & 0.882 & 3.443 & - & - & Yes \\
\hline Stripped Low Nonlocking 4 & 50 & 0.861 & 3.729 & - & - & 0.815 & 3.627 & - & - & Yes \\
\hline Stripped Low Nonlocking 5 & 100 & 0.879 & 2.627 & 6.740 & - & 0.833 & 2.525 & 6.515 & - & Yes \\
\hline Stripped Low Nonlocking 6 & 50 & 1.441 & 4.089 & - & - & 1.395 & 3.987 & - & - & Yes \\
\hline Stripped Low Nonlocking 7 & 25 & 2.116 & - & - & - & 2.070 & - & - & - & Yes \\
\hline Stripped Low Nonlocking 8 & 25 & 1.774 & - & - & - & 1.728 & - & - & - & Yes \\
\hline Stripped Low Nonlocking 9 & 50 & 0.885 & 2.686 & - & - & 0.839 & 2.584 & - & - & Yes \\
\hline Stripped Low Nonlocking 10 & 50 & 0.963 & 2.877 & - & - & 0.917 & 2.775 & - & - & Yes \\
\hline
\end{tabular}


Table XXVIII: Displacement per Load Level Medium Density CaP

\begin{tabular}{|c|c|c|c|c|c|c|c|c|c|c|}
\hline \multirow[b]{2}{*}{ Trial } & \multirow[b]{2}{*}{ Load at failure $(\mathrm{N})$} & \multicolumn{4}{|c|}{ Averaged Displacement (mm) } & \multicolumn{4}{|c|}{ Adjusted Displacement (mm) } & \multirow[b]{2}{*}{ Failed? } \\
\hline & & $25 \mathrm{~N}$ & $50 \mathrm{~N}$ & $100 \mathrm{~N}$ & $200 \mathrm{~N}$ & $25 \mathrm{~N}$ & $50 \mathrm{~N}$ & $100 \mathrm{~N}$ & $200 \mathrm{~N}$ & \\
\hline Large Medium Locking 1 & 200 & 0.259 & 0.589 & 1.282 & 4.219 & 0.213 & 0.487 & 1.057 & 3.696 & Yes \\
\hline Large Medium Locking 2 & 200 & 0.265 & 0.482 & 0.876 & 1.733 & 0.219 & 0.379 & 0.652 & 1.210 & No \\
\hline Large Medium Locking 3 & 200 & 0.169 & 0.359 & 0.707 & 1.476 & 0.123 & 0.257 & 0.482 & 0.954 & No \\
\hline Large Medium Locking 4 & 200 & 0.236 & 0.366 & 0.676 & 1.490 & 0.190 & 0.264 & 0.451 & 0.968 & No \\
\hline Large Medium Locking 5 & 200 & 0.222 & 0.463 & 0.882 & 1.693 & 0.176 & 0.361 & 0.657 & 1.170 & No \\
\hline Large Medium Locking 6 & 200 & 0.132 & 0.275 & 0.505 & 1.131 & 0.086 & 0.172 & 0.281 & 0.609 & No \\
\hline Large Medium Locking 7 & 200 & 0.121 & 0.291 & 0.580 & 1.707 & 0.075 & 0.189 & 0.356 & 1.184 & No \\
\hline Large Medium Locking 8 & 200 & 0.124 & 0.282 & 0.607 & 1.227 & 0.078 & 0.180 & 0.382 & 0.704 & No \\
\hline Large Medium Locking 9 & 200 & 0.184 & 0.360 & 0.669 & 2.058 & 0.138 & 0.258 & 0.444 & 1.535 & No \\
\hline Large Medium Locking 10 & 200 & 0.312 & 0.691 & 1.146 & 1.772 & 0.266 & 0.589 & 0.921 & 1.249 & No \\
\hline Large Medium Nonlocking 1 & 200 & 0.313 & 0.581 & 1.227 & 8.207 & 0.267 & 0.479 & 1.002 & 7.685 & Yes \\
\hline Large Medium Nonlocking 2 & 200 & 0.332 & 0.599 & 1.295 & 7.234 & 0.286 & 0.497 & 1.070 & 6.711 & Yes \\
\hline Large Medium Nonlocking 3 & 200 & 0.367 & 0.678 & 1.421 & 7.612 & 0.321 & 0.576 & 1.196 & 7.090 & Yes \\
\hline Large Medium Nonlocking 4 & 200 & 0.410 & 0.623 & 1.355 & 6.346 & 0.364 & 0.521 & 1.130 & 5.824 & Yes \\
\hline Large Medium Nonlocking 5 & 200 & 0.367 & 0.616 & 1.155 & 7.425 & 0.321 & 0.513 & 0.931 & 6.903 & Yes \\
\hline Large Medium Nonlocking 6 & 200 & 0.465 & 0.721 & 1.286 & 7.491 & 0.419 & 0.619 & 1.061 & 6.968 & Yes \\
\hline Large Medium Nonlocking 7 & - & - & - & - & - & - & - & - & - & - \\
\hline Large Medium Nonlocking 8 & - & - & - & - & - & - & - & - & - & - \\
\hline Large Medium Nonlocking 9 & - & - & - & - & - & - & - & - & - & - \\
\hline Large Medium Nonlocking 10 & - & - & - & - & - & - & - & - & - & - \\
\hline Stripped Medium Locking 1 & 50 & 0.270 & 2.423 & - & - & 0.224 & 2.321 & - & - & Yes \\
\hline Stripped Medium Locking 2 & 200 & 0.279 & 0.502 & 1.028 & 2.984 & 0.233 & 0.399 & 0.803 & 2.461 & Yes \\
\hline Stripped Medium Locking 3 & 200 & 0.271 & 0.467 & 0.813 & 2.963 & 0.225 & 0.365 & 0.588 & 2.440 & Yes \\
\hline Stripped Medium Locking 4 & 200 & 0.369 & 0.634 & 1.037 & 2.322 & 0.323 & 0.532 & 0.812 & 1.799 & No \\
\hline Stripped Medium Locking 5 & 200 & 0.418 & 0.808 & 1.380 & 3.636 & 0.372 & 0.706 & 1.155 & 3.114 & Yes \\
\hline Stripped Medium Locking 6 & 200 & 0.243 & 0.446 & 1.041 & 2.973 & 0.197 & 0.344 & 0.816 & 2.450 & Yes \\
\hline Stripped Medium Locking 7 & 200 & 0.374 & 0.711 & 1.269 & 3.530 & 0.328 & 0.609 & 1.044 & 3.008 & Yes \\
\hline Stripped Medium Locking 8 & 200 & 0.236 & 0.481 & 1.039 & 4.661 & 0.190 & 0.379 & 0.815 & 4.138 & Yes \\
\hline Stripped Medium Locking 9 & 200 & 0.275 & 0.590 & 1.284 & 3.022 & 0.229 & 0.488 & 1.059 & 2.499 & No \\
\hline Stripped Medium Locking 10 & 200 & 0.521 & 0.935 & 1.547 & 3.282 & 0.475 & 0.833 & 1.322 & 2.759 & No \\
\hline Stripped Medium Nonlocking 1 & 100 & 0.520 & 1.011 & 3.575 & - & 0.474 & 0.909 & 3.351 & - & Yes \\
\hline Stripped Medium Nonlocking 2 & 200 & 0.323 & 0.552 & 1.706 & - & 0.277 & 0.450 & 1.481 & - & Yes \\
\hline Stripped Medium Nonlocking 3 & 200 & 0.421 & 0.805 & 1.924 & 8.090 & 0.375 & 0.703 & 1.699 & 7.568 & Yes \\
\hline Stripped Medium Nonlocking 4 & 200 & 0.323 & 0.551 & 1.172 & 8.138 & 0.277 & 0.449 & 0.948 & 7.615 & Yes \\
\hline Stripped Medium Nonlocking 5 & 200 & 0.346 & 0.602 & 1.455 & 7.823 & 0.300 & 0.500 & 1.231 & 7.300 & Yes \\
\hline Stripped Medium Nonlocking 6 & 200 & 0.383 & 0.732 & 1.670 & 8.852 & 0.337 & 0.630 & 1.446 & 8.330 & Yes \\
\hline Stripped Medium Nonlocking 7 & 200 & 0.434 & 0.924 & 2.062 & - & 0.388 & 0.822 & 1.838 & - & Yes \\
\hline Stripped Medium Nonlocking 8 & 200 & 0.357 & 0.651 & 1.447 & 8.260 & 0.311 & 0.549 & 1.222 & 7.737 & Yes \\
\hline Stripped Medium Nonlocking 9 & 200 & 0.467 & 0.872 & 2.328 & - & 0.421 & 0.770 & 2.103 & - & Yes \\
\hline Stripped Medium Nonlocking 10 & 100 & 0.572 & 1.123 & 3.770 & - & 0.526 & 1.021 & 3.545 & - & Yes \\
\hline
\end{tabular}


Table XXIX: Displacement per Load Level High Density CaP

\begin{tabular}{|c|c|c|c|c|c|c|c|c|c|c|}
\hline \multirow[b]{2}{*}{ Trial } & \multirow[b]{2}{*}{ Load at failure $(\mathrm{N})$} & \multicolumn{4}{|c|}{ Averaged Displacement (mm) } & \multicolumn{4}{|c|}{ Adjusted Displacement (mm) } & \multirow[b]{2}{*}{ Failed? } \\
\hline & & $25 \mathrm{~N}$ & $50 \mathrm{~N}$ & $100 \mathrm{~N}$ & $200 \mathrm{~N}$ & $25 \mathrm{~N}$ & $50 \mathrm{~N}$ & $100 \mathrm{~N}$ & $200 \mathrm{~N}$ & \\
\hline Large High Locking 1 & 200 & 0.206 & 0.399 & 0.805 & 4.911 & 0.160 & 0.297 & 0.581 & 4.388 & Yes \\
\hline Large High Locking 2 & 100 & 0.219 & 0.360 & 1.885 & - & 0.173 & 0.258 & 1.660 & - & Yes \\
\hline Large High Locking 3 & 200 & 0.119 & 0.274 & 0.712 & 5.030 & 0.073 & 0.172 & 0.487 & 4.507 & Yes \\
\hline Large High Nonlocking 1 & 100 & 0.390 & 1.107 & 6.440 & - & 0.344 & 1.004 & 6.215 & - & Yes \\
\hline Large High Nonlocking 2 & 200 & 0.483 & 0.885 & 1.505 & 7.794 & 0.437 & 0.783 & 1.280 & 7.272 & Yes \\
\hline Large High Nonlocking 3 & 200 & 0.496 & 0.875 & 1.573 & 8.573 & 0.450 & 0.773 & 1.348 & 8.050 & Yes \\
\hline Stripped High Locking 1 & 200 & 0.239 & 0.411 & 0.687 & 4.051 & 0.193 & 0.308 & 0.462 & 3.528 & Yes \\
\hline Stripped High Locking 2 & 200 & 0.206 & 0.360 & 1.959 & - & 0.160 & 0.258 & 1.735 & - & Yes \\
\hline Stripped High Locking 3 & 200 & 0.227 & 0.395 & 0.679 & 4.022 & 0.181 & 0.293 & 0.455 & 3.499 & Yes \\
\hline Stripped High Locking 4 & 200 & 0.239 & 0.405 & 0.696 & 3.832 & 0.193 & 0.303 & 0.471 & 3.310 & Yes \\
\hline Stripped High Locking 5 & 200 & 0.220 & 0.378 & 0.659 & 1.566 & 0.174 & 0.276 & 0.434 & 1.044 & No \\
\hline Stripped High Locking 6 & 200 & 0.226 & 0.323 & 0.572 & 1.101 & 0.180 & 0.221 & 0.347 & 0.578 & No \\
\hline Stripped High Locking 7 & 200 & 0.217 & 0.326 & 0.582 & 1.052 & 0.171 & 0.224 & 0.357 & 0.529 & No \\
\hline Stripped High Locking 8 & 100 & 0.252 & 0.477 & 3.357 & - & 0.206 & 0.375 & 3.132 & - & Yes \\
\hline Stripped High Locking 9 & 100 & 0.180 & 0.372 & 3.504 & - & 0.134 & 0.270 & 3.280 & - & Yes \\
\hline Stripped High Locking 10 & 100 & 0.243 & 0.442 & 3.600 & - & 0.197 & 0.340 & 3.375 & - & Yes \\
\hline Stripped High Nonlocking 1 & 200 & 0.430 & 0.756 & 1.608 & 7.368 & 0.384 & 0.654 & 1.384 & 6.845 & Yes \\
\hline Stripped High Nonlocking 2 & 50 & 0.548 & 6.581 & - & - & 0.502 & 6.479 & - & - & Yes \\
\hline Stripped High Nonlocking 3 & 50 & 0.456 & 2.818 & - & - & 0.410 & 2.715 & - & - & Yes \\
\hline Stripped High Nonlocking 4 & 200 & 0.635 & 1.105 & 2.257 & 7.386 & 0.589 & 1.003 & 2.032 & 6.863 & Yes \\
\hline Stripped High Nonlocking 5 & 200 & 0.430 & 0.760 & 1.363 & 6.941 & 0.384 & 0.658 & 1.138 & 6.419 & Yes \\
\hline Stripped High Nonlocking 6 & 25 & 0.666 & - & - & - & 0.620 & - & - & - & Yes \\
\hline Stripped High Nonlocking 7 & 100 & 0.807 & 1.280 & 6.318 & - & 0.761 & 1.178 & 6.093 & - & Yes \\
\hline Stripped High Nonlocking 8 & 100 & 0.404 & 0.756 & 6.918 & - & 0.358 & 0.654 & 6.693 & - & Yes \\
\hline Stripped High Nonlocking 9 & 25 & 0.822 & - & - & - & 0.776 & - & - & - & Yes \\
\hline Stripped High Nonlocking 10 & 100 & 0.514 & 0.903 & 8.578 & & 0.468 & 0.801 & 8.353 & - & Yes \\
\hline
\end{tabular}




\section{LIST OF REFERENCES}

1. Cartner JL, Petteys T, Tornetta P 3rd. Mechanical effects of off-axis insertion of locking screws: should we do it? Journal of Orthopaedic Trauma. 28 Supplement 1:S2-S5, April 2014.

2. Ricci William M., Tornetta Paul, Borrelli Joseph Jr. Lag Screw Fixation of Medial Malleolar Fractures: A Biomechanical, Radiographic, and Clinical Comparison of Unicortical Partially Threaded Lag Screws and Bicortical Fully Threaded Lag Screws. Journal of Orthopaedic Trauma. 26(10):602-606, October 2012.

3. Ricci William M, Tornetta Paul III, Petteys Timothy, A Comparison of Screw Insertion Torque and Pullout Strength. Journal of Orthopaedic Trauma. 24(6):374-378, June 2010.

4. Yan L, Chang Z, Xu Z, Liu T, He B, Hao D. Biomechanical effects of bone cement volume on the endplates of augmented vertebral body: a three-dimensional finite element analysis. Chinese Medical Journal (English Edition). Chinese Medical Journal (English Edition). 127(1):79-84, January 5, 2014.

5. Frich LH, Jensen NC. Bone properties of the humeral head and resistance to screw cutout. International Journal of Shoulder Surgery. 2014;8(1):21-26. doi:10.4103/09736042.131851.

6. Basafa E, Murphy RJ, Kutzer MD, Otake Y, Armand M. A Particle Model for Prediction of Cement Infiltration of Cancellous Bone in Osteoporotic Bone Augmentation. Engler AJ, ed. PLoS ONE. 2013;8(6):e67958. doi:10.1371/journal.pone.0067958.

7. Amendola L., Gasbarrini A., Fosco M., et al. Fenestrated pedicle screws for cement-augmented purchase in patients with bone softening: a review of 21 cases. Journal of Orthopaedics and Traumatology.2011;12(4):193-199.

8. Yamana K, Tanaka M, Sugimoto Y, Takigawa T, Ozaki T, Konishi H. Clinical application of a pedicle nail system with polymethylmethacrylate for osteoporotic vertebral fracture. European Spine Journal. 2010;19(10):1643-1650. doi:10.1007/s00586-010-1402-1.

9. Amirfeyz R, Bannister G. The effect of bone porosity on the shear strength of the bone-cement interface. International Orthopaedics. 2009;33(3):843-846. doi:10.1007/s00264-008-0558-3.

10. Flahiff C, Gober G, Nicholas R. Pullout strength of fixation screws from polymethylmethacrylate bone cement. Biomechanics Laboratory, Department of Orthopaedic Surgery, University of Arkansas for Medical Sciences, 22 Dec 1999.

11. Helito C, Bonadio M, Demange M. Screw Loosening and iliotibial band friction after posterolateral corner reconstruction. Department of Orthopaedics and Traumatology, IOTHCFMUSP (Institute of Orthopedics and Traumatology-Hospital and Clinics, Faculty of Medicine, University of São Paulo), São Paulo, Brazil. 13 Mar 2014.

12. Yuan Q, Zhang G, Wu J, Xing Y, Sun Y, Tian W. Clinical evaluation of the polymethylmethacrylate-augmented thoracic and lumbar pedicle screw fixation guided by the three-dimensional navigation for the osteoporosis patients. European Spine Journal, Volume 24, Issue 5, pp 1043-1050, May 2015.

13. Kralinger F, Blauth M, Goldhahn J, Käch K, Voigt C, Platz A, Hanson B. The influence of local bone density on the outcome of one hundred and fifty proximal humeral fractures treated with a locking plate. J Bone Joint Surg Am. 2014 Jun 18;96(12):1026-1032.

14. Stadelmann V, Bretton E, Terrier A, Procter P, Pioletti D. Calcium phosphate cement augmentation of cancellous bone screws can compensate for the absence of cortical fixation. Journal of Biomechanics 43 (2010) 2869-2874, 21 July 2010.

15. Larsson S, Stadelmann V, Arnoldi J, Behrens M, Hess B, Procter P, Murphy M, Pioletti D. Injectable calcium phosphate cement for augmentation around cancellous bone screws. In vivo biomechanical studies. Journal of Biomechanics 45 (2012) 1156 - 1160. Feb 2012.

16. Nowak TE, Burkhart KJ, Andres T, et al. Locking-plate osteosynthesis versus intramedullary nailing for fixation of olecranon fractures: a biomechanical study.International Orthopaedics. 2013;37(5):899-903.

17. Frich LH, Jensen NC. Bone properties of the humeral head and resistance to screw cutout. International Journal of Shoulder Surgery. 2014;8(1):21-26.

18. Lenz M, Windolf M, Mückley T, et al. The locking attachment plate for proximal fixation of periprosthetic femur fractures - a biomechanical comparison of two techniques. International Orthopaedics. 2012;36(9):1915-1921. 
19. Voigt C, Rank C, Waizner K, et al. Biomechanical testing of a new plate system for the distal humerus compared to two well-established implants.International Orthopaedics. 2013;37(4):667-672.

20. Yang S, Kuo S, Chang S, Su T, Chen H, Renn J, Lin T. Biomechanical Comparison of Axial Load Between Cannulated Locking Screws and Noncannulated Cortical Locking Screws.ORTHOPEDICS. 2013; 36: e1316-e1321. doi: 10.3928/01477447-20130920-26.

21. D. Wähnert, J. H. Lange, M. Schulze, et al. A laboratory investigation to assess the influence of cement augmentation of screw and plate fixation in a simulation of distal femoral fracture of osteoporotic and nonosteoporotic bone. Bone Joint J 2013;95-B:1406-9.

22. Grawe B, Le T, Williamson S, Archdeacon A, Zardiackas L. Fracture fixation with two locking screws versus three non-locking screws: A biomechanical comparison in a normal and an osteoporotic bone model. Bone \& Joint Research. 2012;1(6):118-124. doi:10.1302/20463758.16.2000078.

23. Barr C, Behn AW, Yao J. Plating of metacarpal fractures with locked or nonlocked screws, a biomechanical study: how many cortices are really necessary? Hand (New York, NY). 2013;8(4):454-459. doi:10.1007/s11552-013-9544-3.

24. Gradl G, Knobe M, Stoffel M, Prescher A, Dirrichs T, Pape H. Biomechanical evaluation of locking plate fixation of proximal humeral fractures augmented with calcium phosphate cement. J Orthop Trauma 2013;27:399 - 404. Oct 2012.

25. Bariteau J, Fantry A, Blankenhorn B, Lareau C, Paller D, DiGiovanni C. A biomechanical evaluation of locked plating for distal fibula fractures in an osteoporotic sawbone model. Foot and Ankle Surgery 20 (2014) $44-47$. Oct 2013.

26. Kumar S, Gattumeedhi SR, Sankhla B, Garg A, Ingle E, Dagli N. Comparative evaluation of bite forces in patients after treatment of mandibular fractures with miniplate osteosynthesis and internal locking miniplate osteosynthesis. Journal of International Society of Preventive \& Community Dentistry. 2014;4(Suppl 1):S26-S31. doi:10.4103/2231-0762.144575.

27. The Engineering Toolbox. Internet Source. http://www.engineeringtoolbox.com/area-momentinertia-d_1328.html . July 2015.

28. Beam Deflections: Second Order Method; an online lecture. Colorado engineering, July 2015 http://www.colorado.edu/engineering/CAS/courses.d/Structures.d/IAST.Lect10.d/IAST.Lect1 $0 . p d f$.

29. Lovett T. Learn Easy: Beam Bending Moment and Deflection Tables, an internet source. http://www.learneasy.info/MDME/iTester/get-info/beam-bending.html. June 2015. 


\section{Vita}

Kevin Lancaster has lived in Louisville Kentucky since 2001 and is a graduate of DuPont Manual High School. He holds a bachelor's degree in Bioengineering from the University of Louisville Speed Scientific School and has work experience at the Kentucky Spinal Cord Injury Research Center in Louisville and Boston Scientific Inc. in Spencer, Indiana. He is currently seeking a Master of Engineering degree in Bioengineering. 